\title{
Iron-Catalyzed Ligand Free $\alpha$-Alkylation of Methylene Ketones and $\beta$-Alkylation of Secondary Alcohols Using Primary Alcohols
}

\author{
Anitha Alanthadka, Sourajit Bera, and Debasis Banerjee* \\ Department of Chemistry \\ Laboratory of Catalysis and Organic Synthesis \\ Indian Institute of Technology Roorkee \\ Roorkee-247667, Uttarakhand, India \\ E-mail:dbane.fcy@iitr.ac.in
}

\section{Table of Contents}

Alkylation of propiophenone with benzyl alcohol

${ }^{1} \mathrm{H}$ NMR and ${ }^{13} \mathrm{C}$ NMR spectra for selected compounds 


\section{[1.1] Fe-catalyzed alkylation of propiophenone with benzyl alcohol:}

Table S1: Screening of Fe-catalysts ${ }^{\mathrm{a}}$

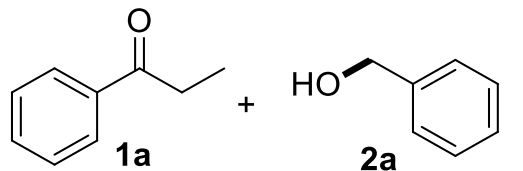

Fe-cat. $(2.5 \mathrm{~mol} \%)$ $t$-BuOK (1.0 equiv.), toluene, $140^{\circ} \mathrm{C}, 24 \mathrm{~h}$<smiles>CC(C[PH2+])C(=O)c1ccccc1</smiles>

\begin{tabular}{|l|l|l|l|}
\hline Entry & Fe-catalyst & $\begin{array}{l}\text { GC-MS conversion } \\
\mathbf{3 a}(\%)\end{array}$ & $\begin{array}{l}\text { Ratio } \\
\mathbf{3 a} / \mathbf{3 a},\end{array}$ \\
\hline $\mathbf{1}^{\mathbf{b}}$ & $\left.\mathbf{F e}_{2}(\mathbf{C O})_{\mathbf{9}} \mathbf{( 2 . 5} \mathbf{~ m o l} \%\right)$ & $\mathbf{8 7}(\mathbf{8 1})$ & $>\mathbf{1 8 : 1}$ \\
\hline 2 & $\mathrm{Fe}(\mathrm{OAc})_{2}(5 \mathrm{~mol} \%)$ & 39 & $0.9: 1$ \\
\hline 3 & $\mathrm{Fe}(\mathrm{acac})_{3}(5 \mathrm{~mol} \%)$ & 35 & $0.6: 1$ \\
\hline 4 & $\mathrm{No} \mathrm{Catalyst}_{5}$ & 24 & $0.6: 1$ \\
\hline $5^{\mathrm{c}}$ & $\mathrm{Fe}_{2}(\mathrm{CO})_{9}(2.5 \mathrm{~mol} \%)$ & 45 & $0.97: 1$ \\
\hline
\end{tabular}

Reaction condition: ${ }^{a}$ Propiophenone $1 \mathrm{1a}(0.5 \mathrm{mmol})$, benzyl alcohol 2a $(0.625 \mathrm{mmol})$, Fe-catalyst $(2.5 \mathrm{~mol} \%)$, $t$-BuOK $(0.5 \mathrm{mmol})$, toluene $(2.0 \mathrm{~mL})$, Schlenk tube under $\mathrm{N}_{2}$ atmosphere, $140{ }^{\circ} \mathrm{C}$ oil bath, $24 \mathrm{~h}$ reaction time. ${ }^{\mathrm{b}}$ Isolated yield (average of two run). ${ }^{\mathrm{c}} t$ - $\mathrm{BuOK}(0.25 \mathrm{mmol})$ was used.

Table S2: Screening of base ${ }^{\mathrm{a}}$
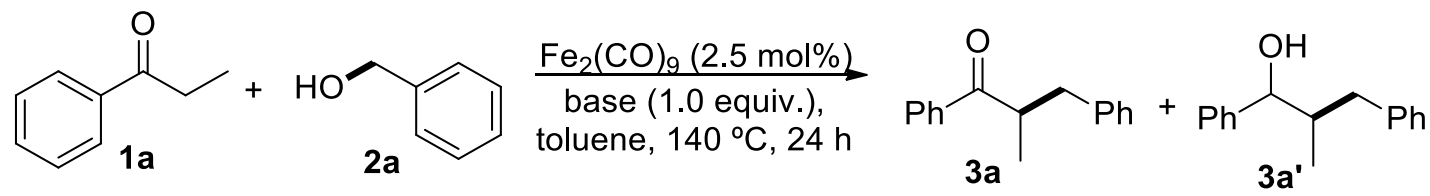

\begin{tabular}{|c|c|c|c|}
\hline Entry & Base & $\begin{array}{c}\text { GC-MS Conversion } \\
\mathbf{3 a}(\%)\end{array}$ & $\begin{array}{r}\text { Ratio } \\
\mathbf{3 a} / \mathbf{3 a}\end{array}$ \\
\hline $\mathbf{1}^{\mathbf{b}}$ & $\boldsymbol{t}$-BuOK & $\mathbf{8 7}(\mathbf{8 1})$ & $>\mathbf{1 8 : 1}$ \\
\hline 2 & $t$-BuONa & 47 & $1.7: 1$ \\
\hline 3 & $\mathrm{Na}_{2} \mathrm{CO}_{3}$ & 6 & - \\
\hline 4 & $\mathrm{~K}_{2} \mathrm{CO}_{3}$ & 9 & - \\
\hline 5 & $\mathrm{~K}_{3} \mathrm{PO}_{4}$ & 5 & - \\
\hline 6 & $\mathrm{No} \mathrm{Base}$ & 8 & - \\
\hline
\end{tabular}

Reaction condition: ${ }^{a}$ Propiophenone 1a $(0.5 \mathrm{mmol})$, benzyl alcohol $2 \mathrm{a}(0.625 \mathrm{mmol}), \mathrm{Fe}_{2}(\mathrm{CO})_{9}(2.5 \mathrm{~mol} \%)$, Base $(0.5 \mathrm{mmol})$, toluene $(2.0 \mathrm{~mL})$, Schlenk tube under $\mathrm{N}_{2}$ atmosphere, $140{ }^{\circ} \mathrm{C}$ oil bath, $24 \mathrm{~h}$ reaction time. ${ }^{b}$ Isolated yield (average of two run).

Table S3: Screening of solvents ${ }^{\mathrm{a}}$<smiles>CCC(=O)c1ccccc1</smiles><smiles>OCc1ccccc1</smiles>

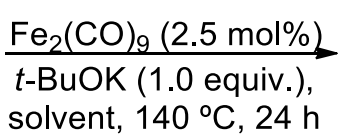
solvent, $140^{\circ} \mathrm{C}, 24 \mathrm{~h}$<smiles>CC(C[PH2+][PH2+])C(=O)c1ccccc1</smiles>

$3 a$

\begin{tabular}{|c|c|c|c|}
\hline Entry & Solvent & $\begin{array}{c}\text { GC-MS Conversion } \\
\mathbf{3 a}(\mathbf{\%})\end{array}$ & $\begin{array}{c}\text { Ratio } \\
\mathbf{3 a} / \mathbf{3 a}\end{array}$ \\
\hline $\mathbf{1}^{\mathbf{b}}$ & Toluene & $\mathbf{8 7}(\mathbf{8 1})$ & $>\mathbf{1 8 : 1}$ \\
\hline 2 & $p$-Xylene & 43 & $1.3: 1$ \\
\hline 3 & $t$-Amyl alcohol & 62 & $2.5: 1$ \\
\hline 4 & DMA & 15 & - \\
\hline
\end{tabular}

Reaction condition: ${ }^{a}$ Propiophenone 1a $(0.5 \mathrm{mmol})$, benzyl alcohol 2a $(0.625 \mathrm{mmol}), \mathrm{Fe}_{2}(\mathrm{CO})_{9}(2.5 \mathrm{~mol} \%)$, $t$-BuOK $(0.5 \mathrm{mmol})$, solvent $(\mathbf{2 . 0} \mathbf{~ m L})$, Schlenk tube under $\mathrm{N}_{2}$ atmosphere, $140{ }^{\circ} \mathrm{C}$ oil bath, $24 \mathrm{~h}$ reaction time. ${ }^{\mathrm{b}}$ Isolated yield (average of two run). 
Table S4: Screening of benzyl alcohol equivalents ${ }^{\mathrm{a}}$<smiles>CCC(=O)c1ccccc1</smiles>

$\mathrm{Fe}_{2}(\mathrm{CO})_{9}(2.5 \mathrm{~mol} \%)$ $t$-BuOK (1.0 equiv.), toluene, $140^{\circ} \mathrm{C}, 24 \mathrm{~h}$<smiles>CC(C[PH2+])C(=O)c1ccccc1</smiles>

\begin{tabular}{|c|c|c|c|}
\hline Entry & $\begin{array}{c}\text { Benzyl alcohol (X } \\
\text { equiv.) }\end{array}$ & $\begin{array}{c}\text { GC-MS Conversion } \\
\mathbf{3 a}(\%)\end{array}$ & $\begin{array}{c}\text { Ratio } \\
\mathbf{3 a} / \mathbf{3 a}\end{array}$ \\
\hline 1 & 1.0 & 79 & $7.9: 1$ \\
\hline $\mathbf{2}^{\mathbf{b}}$ & $\mathbf{1 . 2 5}$ & $\mathbf{8 7}(\mathbf{8 1})$ & $>\mathbf{1 8 : 1}$ \\
\hline 3 & 1.5 & 85 & $8.5: 1$ \\
\hline
\end{tabular}

Reaction condition: ${ }^{a}$ Propiophenone 1 a $(0.5 \mathrm{mmol})$, benzyl alcohol 2 a ( $\mathrm{X}$ equiv.), $\mathrm{Fe}_{2}(\mathrm{CO})_{9}(2.5 \mathrm{~mol} \%), t$ $\operatorname{BuOK}(0.5 \mathrm{mmol})$, toluene $(2.0 \mathrm{~mL})$, Schlenk tube under $\mathrm{N}_{2}$ atmosphere, $140{ }^{\circ} \mathrm{C}$ oil bath, $24 \mathrm{~h}$ reaction time. ${ }^{\mathrm{b}}$ Isolated yield (average of two run).

Table S5: Screening of temperature ${ }^{\mathrm{a}}$

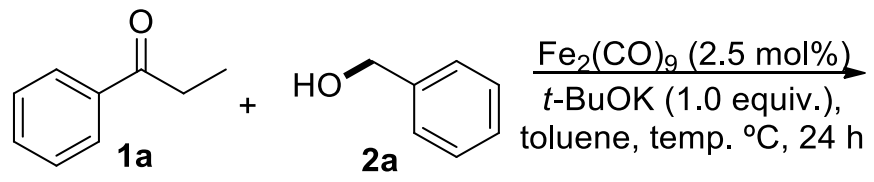<smiles>CC(C[PH2+])C(=O)c1ccccc1</smiles>

\begin{tabular}{|c|c|c|c|}
\hline Entry & Temp. $^{\circ} \mathbf{C}$ & $\begin{array}{c}\text { GC-MS Conversion } \\
\mathbf{3 a}(\%)\end{array}$ & $\begin{array}{r}\text { Ratio } \\
\mathbf{3 a} / \mathbf{3 a}\end{array}$ \\
\hline 1 & 120 & 52 & $1.8: 1$ \\
\hline 2 & 130 & 67 & $2.6: 1$ \\
\hline $\mathbf{3}^{\mathbf{b}}$ & $\mathbf{1 4 0}$ & $\mathbf{8 7}(\mathbf{8 1})^{\mathbf{b}}$ & $>\mathbf{1 8 : 1}$ \\
\hline
\end{tabular}

Reaction condition: ${ }^{\mathrm{a}}$ Propiophenone $1 \mathrm{a}(0.5 \mathrm{mmol})$, benzyl alcohol $2 \mathrm{a}(0.625 \mathrm{mmol}), \mathrm{Fe}_{2}(\mathrm{CO})_{9}(2.5 \mathrm{~mol} \%)$, $t$-BuOK $(0.5 \mathrm{mmol})$, toluene $(2.0 \mathrm{~mL})$, Schlenk tube under $\mathrm{N}_{2}$ atmosphere, Temp ${ }^{\circ} \mathbf{C}$ oil bath, $24 \mathrm{~h}$ reaction time. ${ }^{\mathrm{b}}$ Isolated yield (average of two run). 


\section{[1.2] Deuterium incorporation experiments:}

\section{Scheme S1}<smiles>[2H]C([2H])(O)C([2H])([18OH])[18OH]</smiles>

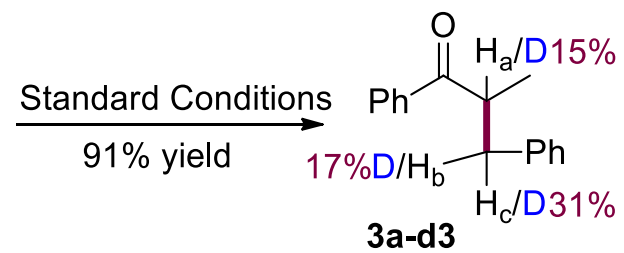

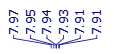

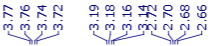

Standard ${ }^{1} \mathrm{H}-\mathrm{NMR}$ spectra of compound $\mathbf{3 a}$

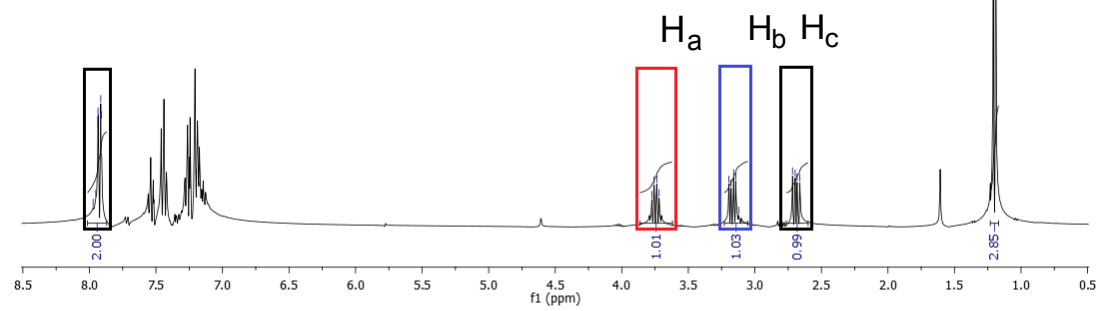

\section{Crude ${ }^{1} \mathrm{H}-\mathrm{NMR}$ spectra of Scheme S1}

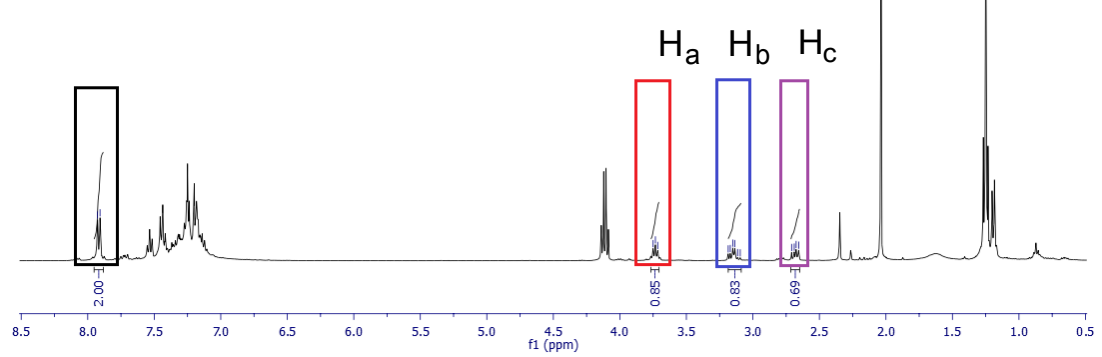

Conversion was calculated by ${ }^{1} \mathrm{H}-\mathrm{NMR}$ integration value

\begin{tabular}{|l|c|c|c|c|}
\hline & & $\begin{array}{c}\text { Deuterium } \\
\text { incorporation in } \\
\mathbf{H}_{\mathbf{a}} \text { Position }\end{array}$ & $\begin{array}{c}\text { Deuterium } \\
\text { incorporation in } \\
\mathbf{H}_{\mathbf{b}} \text { Position }\end{array}$ & $\begin{array}{c}\text { Deuterium } \\
\text { incorporation in } \\
\mathbf{H}_{\mathbf{c}} \text { Position }\end{array}$ \\
\hline $\begin{array}{l}\text { Signal } \delta \\
\text { ppm }\end{array}$ & $\begin{array}{c}7.91-7.97[\mathrm{dd}, \\
\left.\mathrm{CH}_{2},(2 \mathrm{H})\right]\end{array}$ & $3.75(1 \mathrm{H})$ & $3.19(1 \mathrm{H})$ & $2.71(1 \mathrm{H})$ \\
\hline $\begin{array}{l}\text { Integral } \\
\text { Value }\end{array}$ & 2.0 & 0.85 & 0.83 & 0.69 \\
\hline $\begin{array}{l}\text { Calculated } \\
\text { ratio }\end{array}$ & & $\begin{array}{c}(1-0.85) \times 100= \\
\mathbf{1 5 \%}\end{array}$ & $\begin{array}{c}(1-0.83) \times 100= \\
\mathbf{1 7 \%}\end{array}$ & $\begin{array}{c}(1-0.69) \times 100= \\
\mathbf{3 1 \%}\end{array}$ \\
\hline
\end{tabular}




\section{Scheme S2}

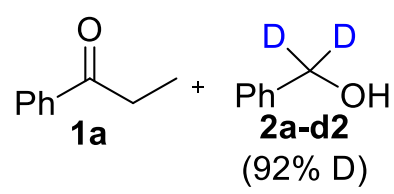

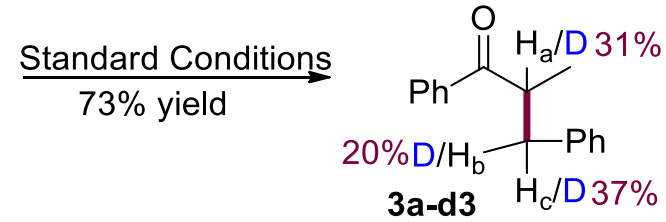

Standard ${ }^{1} \mathrm{H}-\mathrm{NMR}$ spectra of compound $\mathbf{3 a}$

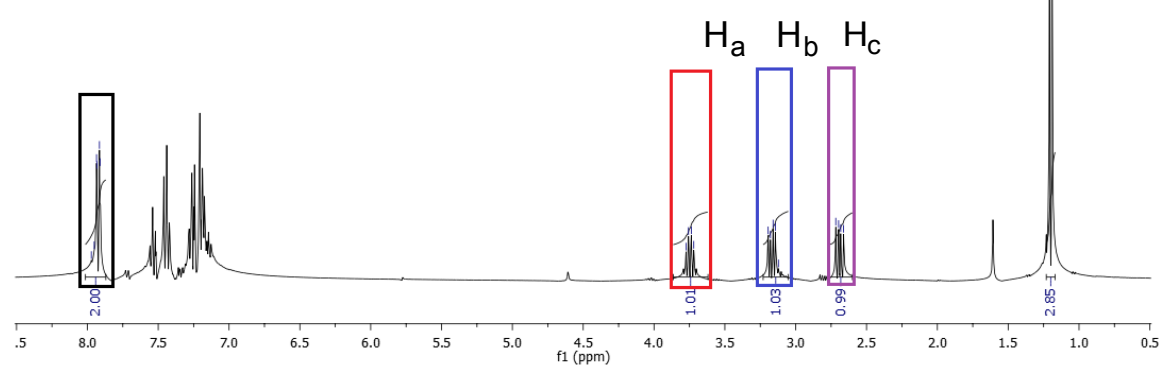

${ }_{8}$ Crude ${ }^{1} \mathrm{H}$-NMR spectra of Scheme S2

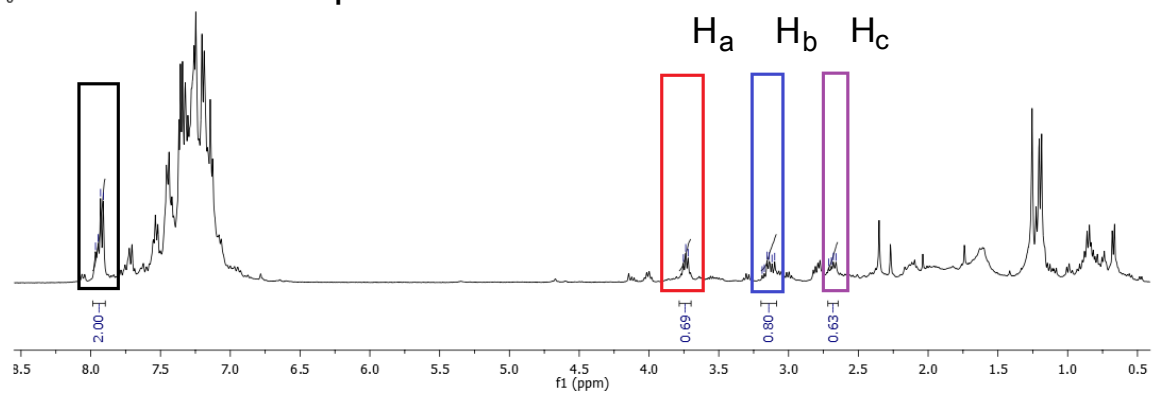

Conversion was calculated by ${ }^{1} \mathrm{H}-\mathrm{NMR}$ integration value

\begin{tabular}{|l|c|c|c|c|}
\hline & & $\begin{array}{c}\text { Deuterium } \\
\text { incorporation in } \\
\mathbf{H}_{\mathbf{a}} \text { Position }\end{array}$ & $\begin{array}{c}\text { Deuterium } \\
\text { incorporation in } \\
\mathbf{H}_{\mathbf{b}} \text { Position }\end{array}$ & $\begin{array}{c}\text { Deuterium } \\
\text { incorporation in } \\
\mathbf{H}_{\mathbf{c}} \text { Position }\end{array}$ \\
\hline $\begin{array}{l}\text { Signal } \delta \\
\text { ppm }\end{array}$ & $\begin{array}{c}7.91-7.97[\mathrm{dd}, \\
\left.\mathrm{CH}_{2},(2 \mathrm{H})\right]\end{array}$ & $3.75(1 \mathrm{H})$ & $3.19(1 \mathrm{H})$ & $2.71(1 \mathrm{H})$ \\
\hline $\begin{array}{l}\text { Integral } \\
\text { Value }\end{array}$ & 2.0 & 0.69 & 0.80 & 0.63 \\
\hline $\begin{array}{l}\text { Calculated } \\
\text { ratio }\end{array}$ & & $\begin{array}{c}(1-0.69) \times 100= \\
\mathbf{3 1 \%}\end{array}$ & $\begin{array}{c}(1-0.80) \times 100= \\
\mathbf{2 0 \%}\end{array}$ & $\begin{array}{c}(1-0.63) \times 100= \\
\mathbf{3 7 \%}\end{array}$ \\
\hline
\end{tabular}




\section{Scheme S3}

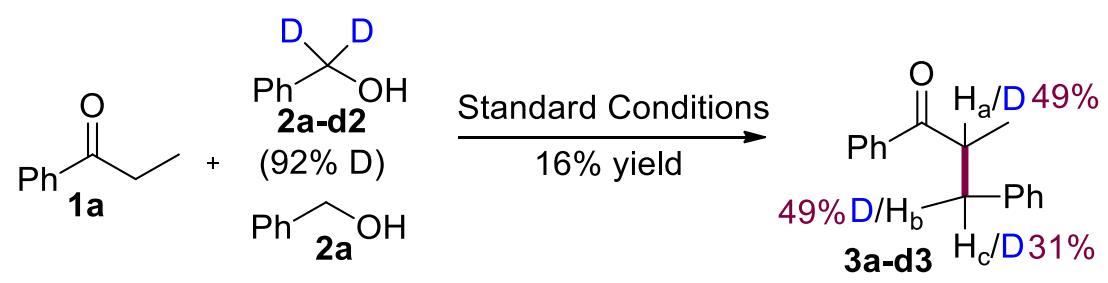

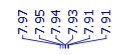

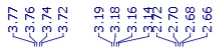

Standard ${ }^{1} \mathrm{H}-\mathrm{NMR}$ spectra of compound $\mathbf{3 a}$
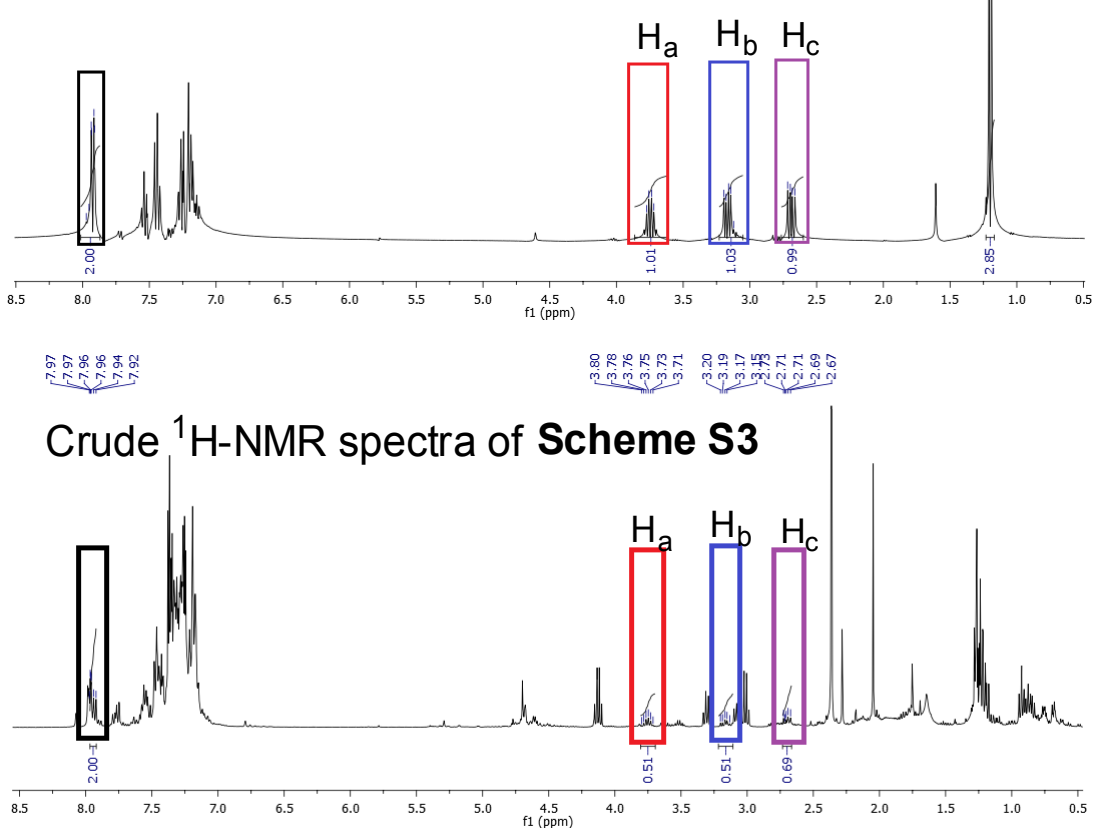

Conversion was calculated by ${ }^{1} \mathrm{H}-\mathrm{NMR}$ integration value

\begin{tabular}{|l|c|c|c|c|}
\hline & & $\begin{array}{c}\text { Deuterium } \\
\text { incorporation in } \\
\mathbf{H}_{\mathbf{a}} \text { Position }\end{array}$ & $\begin{array}{c}\text { Deuterium } \\
\text { incorporation in } \\
\mathbf{H}_{\mathbf{b}} \text { Position }\end{array}$ & $\begin{array}{c}\text { Deuterium } \\
\text { incorporation in } \\
\mathbf{H}_{\mathbf{c}} \text { Position }\end{array}$ \\
\hline $\begin{array}{l}\text { Signal } \delta \\
\text { ppm }\end{array}$ & $\begin{array}{c}7.91-7.97[\mathrm{dd}, \\
\left.\mathrm{CH}_{2},(2 \mathrm{H})\right]\end{array}$ & $3.75(1 \mathrm{H})$ & $3.19(1 \mathrm{H})$ & $2.71(1 \mathrm{H})$ \\
\hline $\begin{array}{l}\text { Integral } \\
\text { Value }\end{array}$ & 2.0 & 0.51 & 0.51 & 0.69 \\
\hline $\begin{array}{l}\text { Calculated } \\
\text { ratio }\end{array}$ & & $(1-0.51) \times 100=$ & $(1-0.51) \times 100=$ & $\begin{array}{c}(1-0.69) \times 100= \\
\mathbf{4 9 \%} \%\end{array}$ \\
\hline
\end{tabular}




\section{Scheme S4}
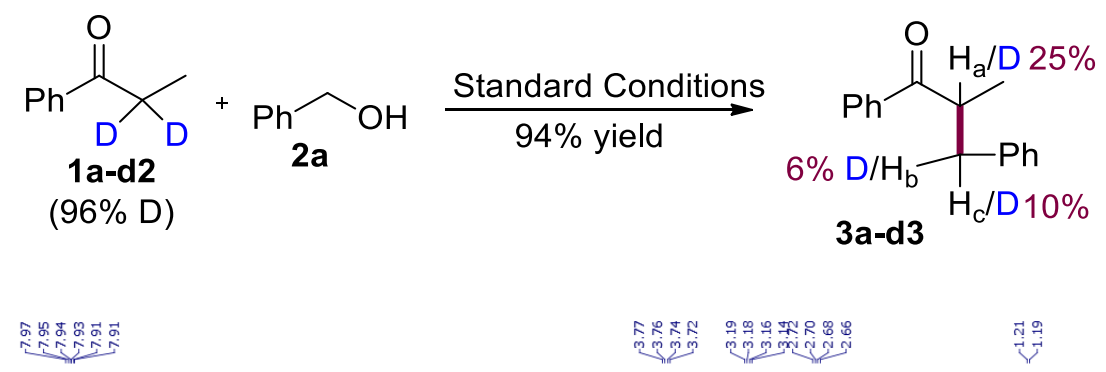

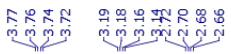

Standard ${ }^{1} \mathrm{H}-\mathrm{NMR}$ spectra of compound $\mathbf{3 a}$

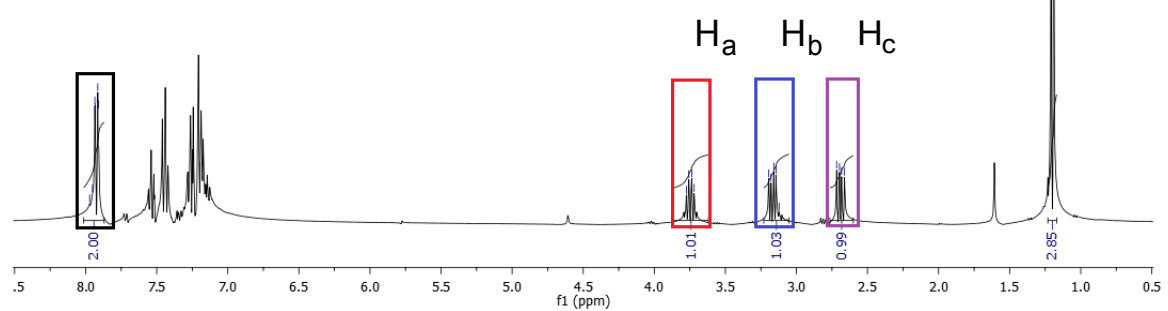

Crude ${ }^{1} \mathrm{H}-\mathrm{NMR}$ spectra of Scheme S4

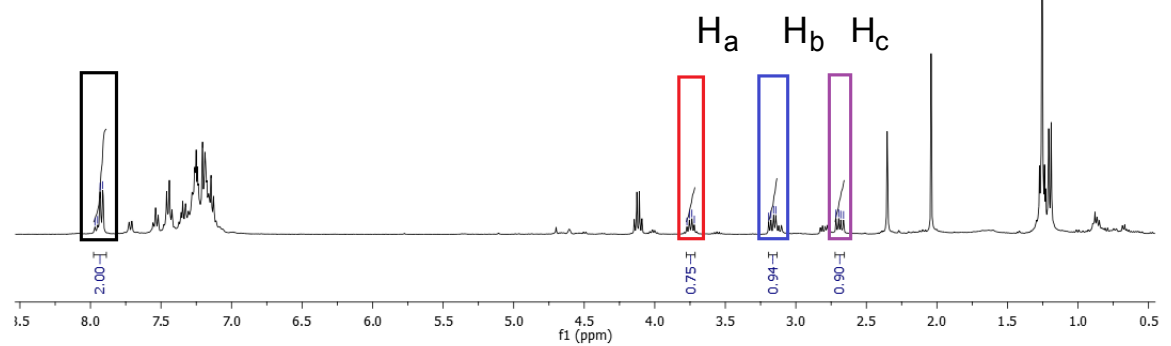

Conversion was calculated by ${ }^{1} \mathrm{H}-\mathrm{NMR}$ integration value

\begin{tabular}{|l|c|c|c|c|}
\hline & & $\begin{array}{c}\text { Deuterium } \\
\text { incorporation in } \\
\mathbf{H}_{\mathbf{a}} \text { Position }\end{array}$ & $\begin{array}{c}\text { Deuterium } \\
\text { incorporation in } \\
\mathbf{H}_{\mathbf{b}} \text { Position }\end{array}$ & $\begin{array}{c}\text { Deuterium } \\
\text { incorporation in } \\
\mathbf{H}_{\mathbf{c}} \text { Position }\end{array}$ \\
\hline $\begin{array}{l}\text { Signal } \delta \\
\text { ppm }\end{array}$ & $\begin{array}{c}7.91-7.97[\mathrm{dd}, \\
\left.\mathrm{CH}_{2},(2 \mathrm{H})\right]\end{array}$ & $3.75(1 \mathrm{H})$ & $3.19(1 \mathrm{H})$ & $2.71(1 \mathrm{H})$ \\
\hline $\begin{array}{l}\text { Integral } \\
\text { Value }\end{array}$ & 2.0 & 0.75 & 0.94 & 0.90 \\
\hline $\begin{array}{l}\text { Calculated } \\
\text { ratio }\end{array}$ & & $\begin{array}{c}(1-0.75) \times 100= \\
\mathbf{2 5 \%}\end{array}$ & $\begin{array}{c}(1-0.94) \times 100= \\
\mathbf{6 \%}\end{array}$ & $\begin{array}{c}(1-0.90) \times 100= \\
\mathbf{1 0 \%}\end{array}$ \\
\hline
\end{tabular}




\section{Scheme S5}

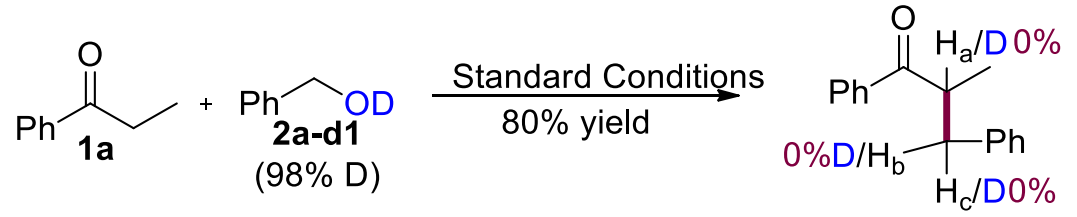

3a

Scheme S6: Determination of rate and order of reaction : Run 1: Reaction was carried out in $2 \mathrm{~mL}$ of toluene and yield was calculated by GC<smiles>CCC(=O)c1ccccc1</smiles>

1a<smiles>OCc1ccccc1</smiles>

2a

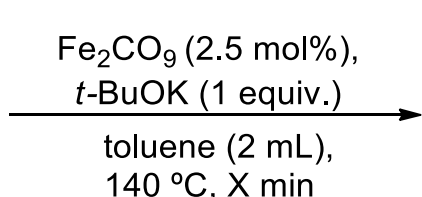

$140^{\circ} \mathrm{C}, \mathrm{X} \min$<smiles>CC(Cc1ccccc1)C(=O)c1ccccc1</smiles>

$3 a$

\begin{tabular}{|c|c|c|c|c|c|}
\hline No. & $\begin{array}{c}\mathbf{1 a} \\
(\mathrm{mmol})\end{array}$ & $\begin{array}{c}\mathbf{2 a} \\
(\mathrm{mmol})\end{array}$ & $\begin{array}{c}\mathrm{Fe}_{2} \mathrm{CO}_{9} \\
(\mathrm{mmol})\end{array}$ & $\begin{array}{c}t \text {-BuOK } \\
(\mathrm{mmol})\end{array}$ & $\begin{array}{c}\text { toluene } \\
(\mathrm{mL})\end{array}$ \\
\hline Run 1 & 0.45 & 0.5625 & 0.01125 & 0.45 & 2.0 \\
\hline
\end{tabular}

\begin{tabular}{|c|c|c|}
\hline Sl. No. & Time (min) & Concentration of 1a (mM) \\
\hline 1 & 60 & 127 \\
\hline 2 & 90 & 112 \\
\hline 3 & 120 & 101 \\
\hline 4 & 150 & 90 \\
\hline 5 & 180 & 78 \\
\hline 6 & 210 & 67 \\
\hline 7 & 240 & 59 \\
\hline 8 & 270 & 54 \\
\hline 9 & 300 & 45 \\
\hline
\end{tabular}


Scheme S7: Determination of rate and order of reaction: Run 2: Reaction was carried out in $2 \mathrm{~mL}$ of toluene and yield was calculated by GC<smiles>CCC(=O)c1ccccc1</smiles>

$1 \mathrm{a}$<smiles>OCc1ccccc1</smiles>

$2 a$

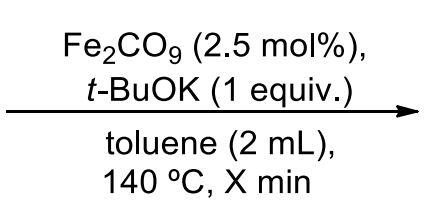

$140^{\circ} \mathrm{C}, \mathrm{X} \min$<smiles>CC(Cc1ccccc1)C(=O)c1ccccc1</smiles>

$3 a$

\begin{tabular}{|c|c|c|c|c|c|}
\hline No. & $\begin{array}{c}\mathbf{1 a} \\
(\mathrm{mmol})\end{array}$ & $\begin{array}{c}\mathbf{2 a} \\
(\mathrm{mmol})\end{array}$ & $\begin{array}{c}\mathrm{Fe}_{2} \mathrm{CO}_{9} \\
(\mathrm{mmol})\end{array}$ & $\begin{array}{c}t \text {-BuOK } \\
(\mathrm{mmol})\end{array}$ & toluene $(\mathrm{mL})$ \\
\hline Run 2 & 0.50 & 0.6125 & 0.0125 & 0.50 & 2.0 \\
\hline
\end{tabular}

\begin{tabular}{|c|c|c|}
\hline Sl. No. & Time (min) & Concentration of 1a (mM) \\
\hline 1 & 60 & 152 \\
\hline 2 & 90 & 142 \\
\hline 3 & 120 & 134 \\
\hline 4 & 150 & 123 \\
\hline 5 & 180 & 108 \\
\hline 6 & 210 & 97 \\
\hline 7 & 240 & 85 \\
\hline 8 & 270 & 75 \\
\hline 9 & 300 & 65 \\
\hline
\end{tabular}

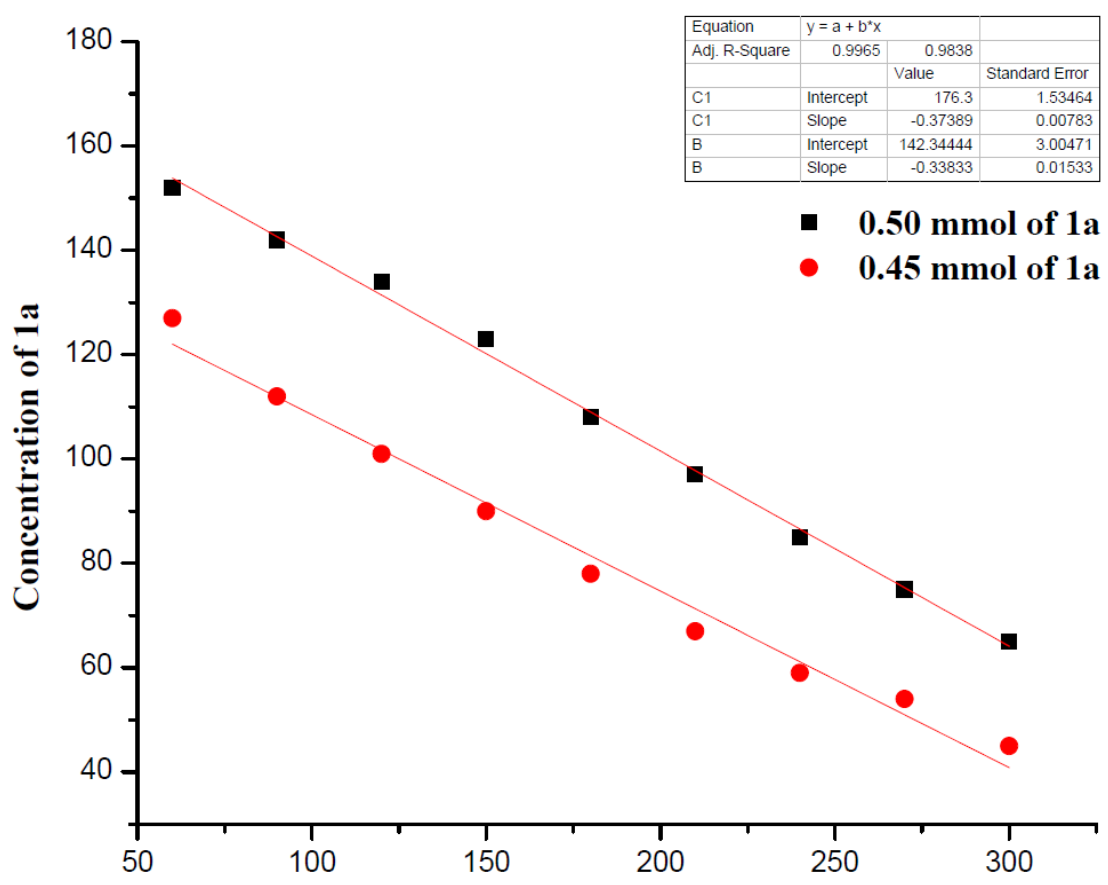

Time (min)

Figure S1: Graphical representation for determination of rate and order of reaction. 
Considering steady state approximation for benzyl alcohol

$$
\begin{aligned}
& \text { From Run 1: } \text { Slope }=k[1 \mathrm{a}]^{\mathrm{x}} \\
& -0.338=k[0.45]^{\mathrm{x}} \\
& \text { From Run 2: Slope }=k[1 \mathrm{a}]^{\mathrm{x}} \\
& -0.373=k[0.50]^{\mathrm{x}} \\
& -0.373 /-0.338=[0.50]^{\mathrm{x}} /[0.45]^{\mathrm{x}} \\
& 1.103=[1.111]^{\mathrm{x}} \\
& \log (1.103)=x \cdot \log (1.111) \\
& \mathrm{x}=0.043 / 0.046 \\
& =0.93 \approx 1 \\
& \text { Rate }=k[1 \mathrm{a}]^{1}
\end{aligned}
$$

\section{Scheme S8: Reaction profile}
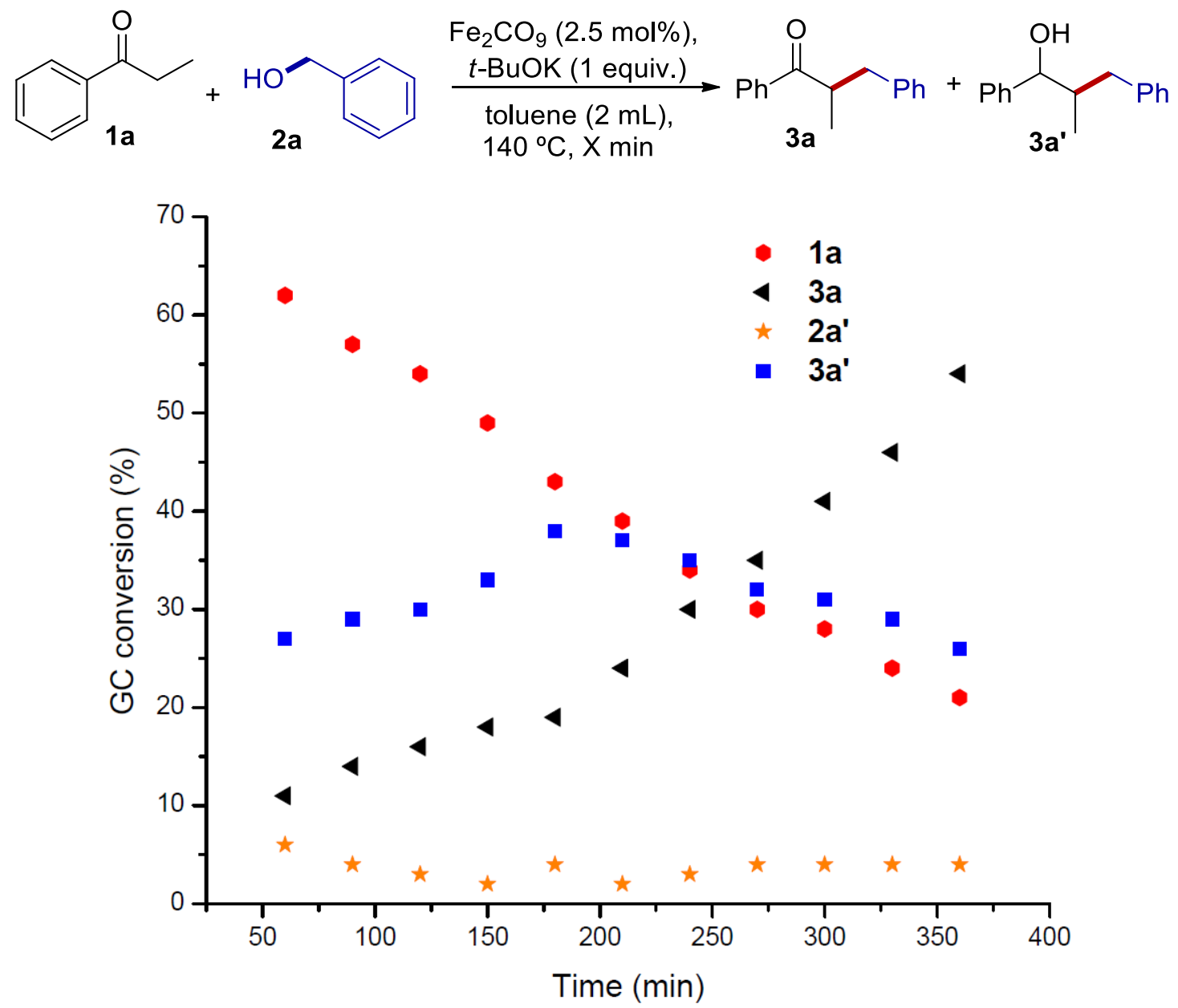

Time-conversion-plot for the reaction of propiophenone (1a) with benzyl alcohol (2a) 
Reaction conditions: Propiophenone $1 \mathrm{a}(0.5 \mathrm{mmol})$, benzyl alcohol 2a $(0.625 \mathrm{mmol}), \mathrm{Fe}_{2}(\mathrm{CO})_{9}(2.5 \mathrm{~mol} \%)$, $t$-BuOK $(0.5 \mathrm{mmol})$, toluene $(2.0 \mathrm{~mL})$, Schlenk tube under $\mathrm{N}_{2}$ atmosphere, $140{ }^{\circ} \mathrm{C}$ oil bath.

\section{Scheme S9: Determination of TON and TOF}

TON Calculation for $\mathbf{3 a}$ :

Product amount $=0.402 \mathrm{mmol}(90 \mathrm{mg})$

Catalyst amount $=0.0125 \mathrm{mmol}$

$\mathrm{TON}=0.402 \mathrm{mmol} / 0.0125 \mathrm{mmol}$

$$
=32
$$

TOF Calculation for $\mathbf{3 a}$ :

$\mathrm{TOF}=\mathrm{TON} /$ Reaction time

$$
\begin{aligned}
& =32 / 24 \mathrm{~h} \\
& =1.33 \mathrm{~h}^{-1}
\end{aligned}
$$

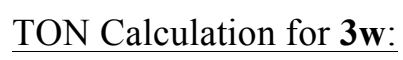

Product amount $=0.457 \mathrm{mmol}(108 \mathrm{mg})$

Catalyst amount $=0.0125 \mathrm{mmol}$

TON $=0.457 \mathrm{mmol} / 0.0125 \mathrm{mmol}$

$=36.6$

TOF Calculation for $\mathbf{3 w}$ :

$\mathrm{TOF}=\mathrm{TON} /$ Reaction time

$=36.6 / 24 \mathrm{~h}$

$=1.52 \mathrm{~h}^{-1}$ 
[1.3] Scheme S10: IR spectroscopic studies

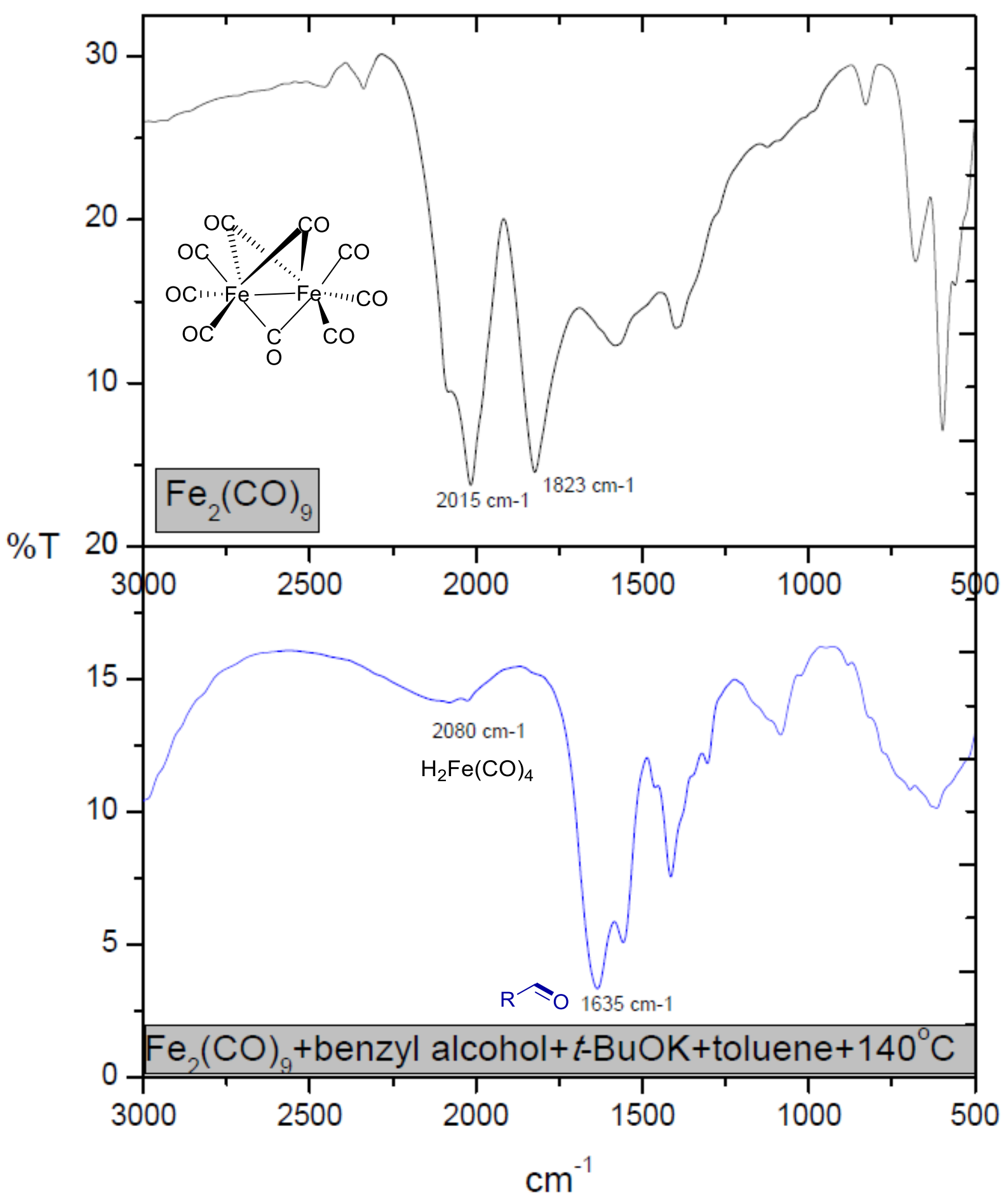

\section{[1.4] Scheme S11: XRD analysis for compound 3j}

White crystals of $\mathbf{3 j}$ were obtained by slow evaporation of the solution from the complexes in acetonitrile. The X-ray data collection and processing for complexes were performed on a Bruker Kappa Apex-II CCD diffractometer by using graphite monochromated Mo-K $\alpha$ 
radiation $(\lambda=0.71073 \AA)$ at $293 \mathrm{~K}$ for $\mathbf{3 j}$. Crystal structures were solved by direct methods. Structure solutions, refinement, and data output were carried out with the SHELXTL program. All non-hydrogen atoms were refined anisotropically. Hydrogen atoms were placed in geometrically calculated positions and refined using a riding model. Images were created with the DIAMOND program.

Identification code: ups3578db_sb1_289_0m_a

CCDC 1895414

Bond precision: $\mathrm{C}-\mathrm{C}=0.0018 \mathrm{~A}$

Wavelength $=0.71073$

Cell: $\mathrm{a}=11.391(2) \mathrm{b}=5.7875(9) \mathrm{c}=24.004(4)$

Alpha $=90$ beta $=93.919(14)$ gamma $=90$

Temperature: $293 \mathrm{~K}$

Calculated Reported

Volume

$1578.8(5)$

1578.8(5)

Space group

P 21/n

P 1 21/n 1

Hall group

-P 2yn

-P 2yn

Moiety formula

C21 H18 O

C21 H18 O

Sum formula

C21 H18 O

C21 H18 O

$\mathrm{Mr}$

286.35

286.35

Dx,g cm-3

1.205

1.205

Z

4

4

$\begin{array}{lll}\mathrm{Mu}(\mathrm{mm}-1) & 0.072 & 0.072\end{array}$ 
608.0

F000'

608.25

h,k,lmax

$18,9,38$

$17,9,38$

Nref

6800

6238

Tmin,Tmax

$0.984,0.984$

$0.985,0.985$

Tmin'

0.984

Correction method $=\#$ Reported T Limits: Tmin $=0.985$ Tmax $=0.985$

AbsCorr $=$ MULTI-SCAN

Data completeness $=0.917 \quad$ Theta $(\max )=34.674$

$\mathrm{R}($ reflections $)=0.0544(2979) \quad \mathrm{wR} 2($ reflections $)=0.1873(6238)$

$\mathrm{S}=0.855 \quad$ Npar $=199$

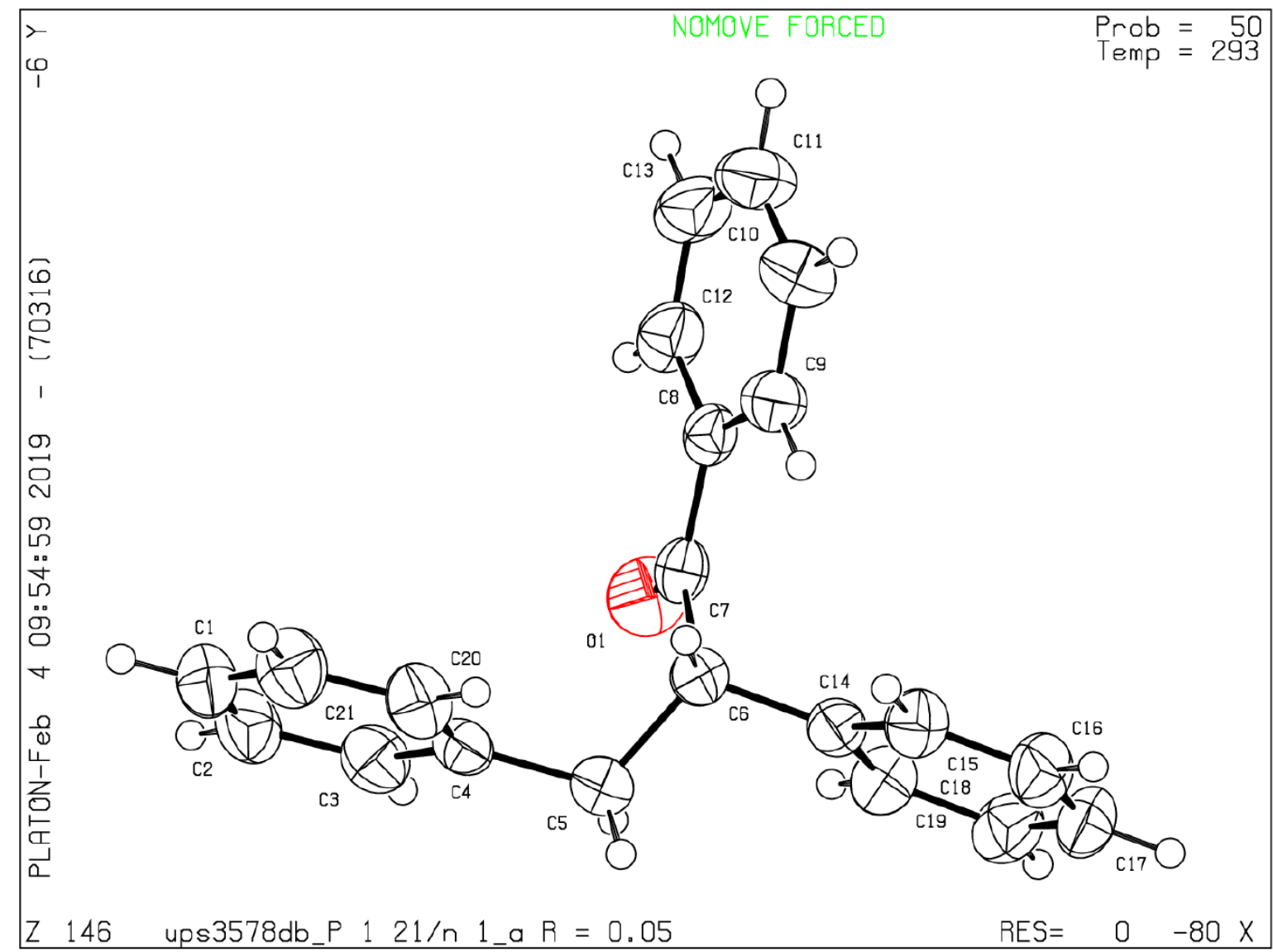

Platon-ellipsoid plot for compound $\mathbf{3 j}$

Figure S2: Crystallographic data for compound $\mathbf{3 j}$ 
Scheme S12: Substrates with poor reactivity profile.

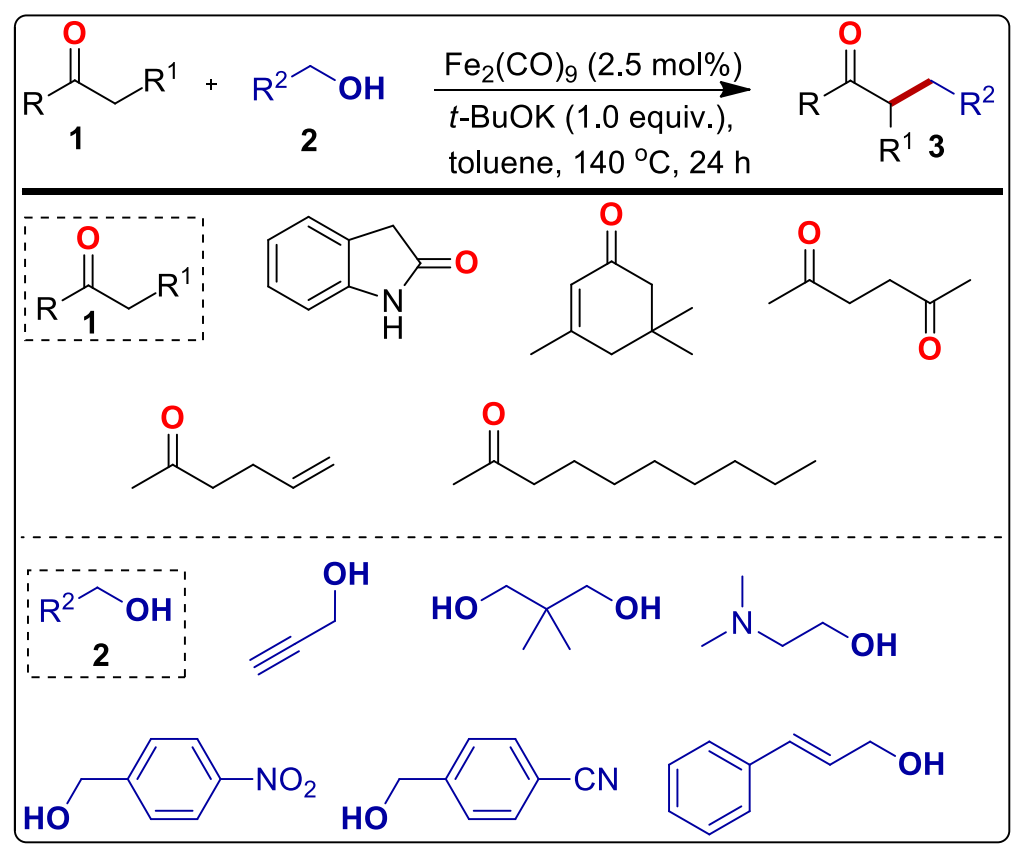

Scheme S13: Determination of deuterium incorporation for deuterated reagents:

\begin{tabular}{|c|c|c|c|}
\hline & & $\begin{array}{c}\text { Deuterium } \\
\text { incorporation in } \\
\mathbf{C H}_{2} \text { position }\end{array}$ & $\begin{array}{c}\text { Deuterium } \\
\text { incorporation in } \\
\text { OH position }\end{array}$ \\
\hline $\begin{array}{c}\text { Signal } \delta \text { ppm } \\
\text { (Standard) }\end{array}$ & $7.24-7.36(5 \mathrm{H})$ & $4.65(2 \mathrm{H})$ & $2.65(1 \mathrm{H})$ \\
\hline $\begin{array}{l}\text { Integral Value } \\
\text { for } \mathbf{2 a - d 2}\end{array}$ & 5.0 & 0.16 & 1.05 \\
\hline Calculated ratio & & $\{(2-0.16) / 2\} \times 100=\mathbf{9 2 \%}$ & $\{(1-1)\} \times 100=\mathbf{0 \%}$ \\
\hline $\begin{array}{l}\text { Integral Value } \\
\text { for } \mathbf{2 a - d} \mathbf{1}\end{array}$ & 5.0 & 2.0 & 0.02 \\
\hline \multirow[t]{2}{*}{ Calculated ratio } & & $\{(2.0-2.0) / 2\} \times 100=\mathbf{0} \%$ & $\{(1-0.02)\} \times 100=\mathbf{9 8 \%}$ \\
\hline & & $\begin{array}{c}\text { Deuterium } \\
\text { incorporation in } \\
\mathbf{C H}_{\mathbf{2}} \text { position }\end{array}$ & $\begin{array}{c}\text { Deuterium } \\
\text { incorporation in } \\
\mathbf{C H}_{\mathbf{3}} \text { position }\end{array}$ \\
\hline $\begin{array}{l}\text { Signal } \delta \text { ppm } \\
\text { (Standard) }\end{array}$ & $7.97-7.42(5 \mathrm{H})$ & $4.65(2 \mathrm{H})$ & $1.20(3 \mathrm{H})$ \\
\hline $\begin{array}{l}\text { Integral Value } \\
\text { for } \mathbf{1 a - d} \mathbf{2}\end{array}$ & 5.0 & 0.06 & 3 \\
\hline Calculated ratio & & $\{2.0-0.06 / 2\} \times 100=\mathbf{9 7 \%}$ & $\{3-3\} \times 100=\mathbf{0} \%$ \\
\hline
\end{tabular}




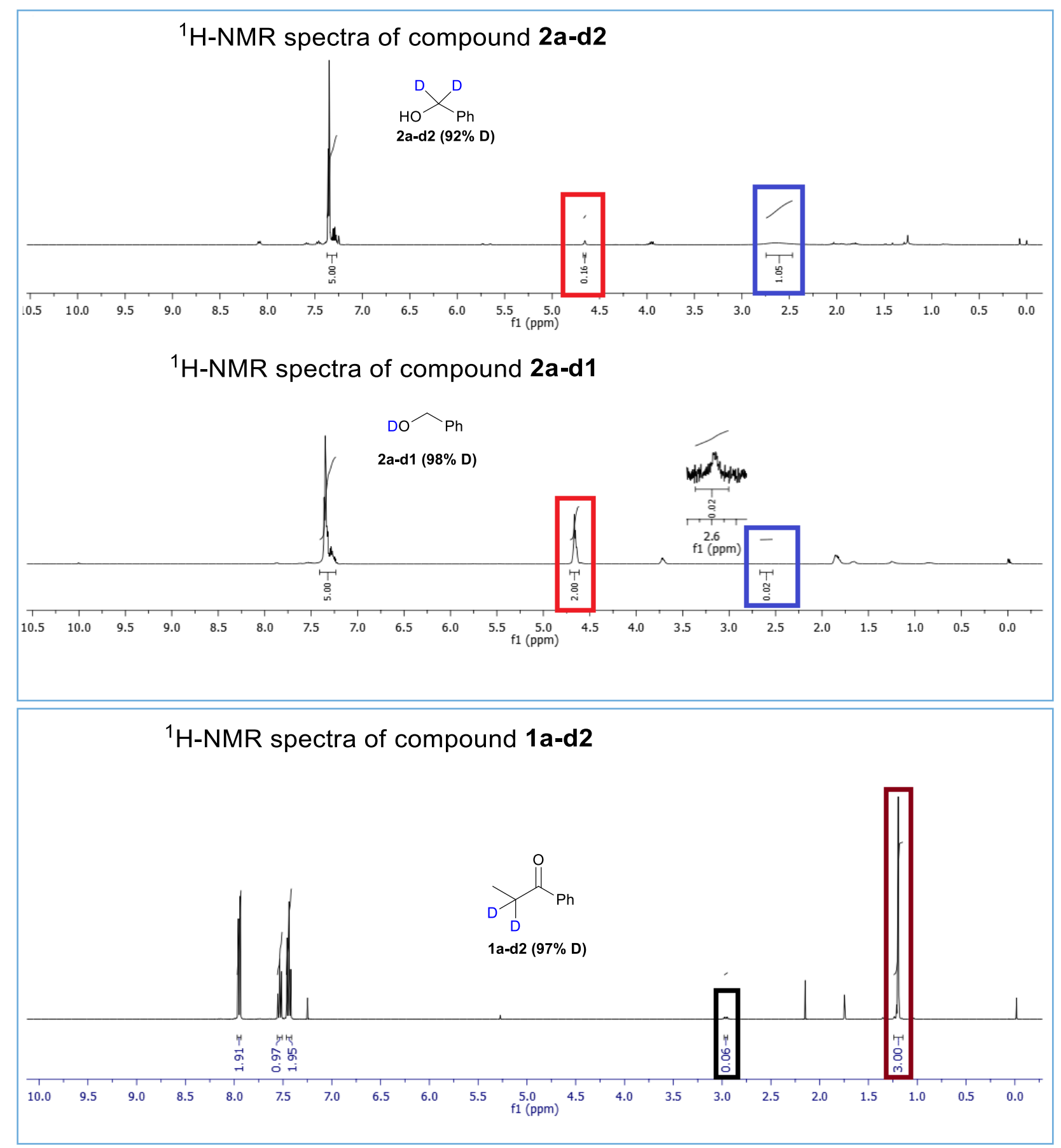


Scheme S14: Detection of $\mathrm{H}_{2}$ gas liberation.

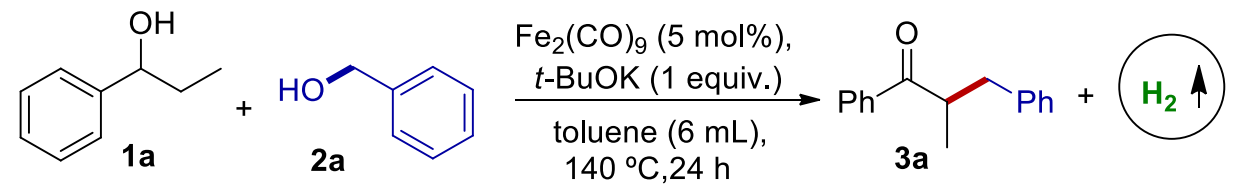

In a $100 \mathrm{~mL}$ oven dried Ace Pressure tube, propiophenone 1a $(3.0 \mathrm{mmol}), \mathrm{Fe}_{2}(\mathrm{CO})_{9}(5$ mol\%), benzyl alcohol 2a $(3.75 \mathrm{mmol})$ and $t$-BuOK $(3.0 \mathrm{mmol})$, were added followed by toluene $6 \mathrm{~mL}$ under an atmosphere of $\mathrm{N}_{2}$ and the reaction mixture was sealed with septum and heated at $140{ }^{\circ} \mathrm{C}$ for $24 \mathrm{~h}$. After completion of reaction the generated $\mathrm{H}_{2}$ gas was collected and detected by Centurion Scientific Gas Chromatograph (CS-5700 $\left.{ }^{+}\right)$through TCD Detector.

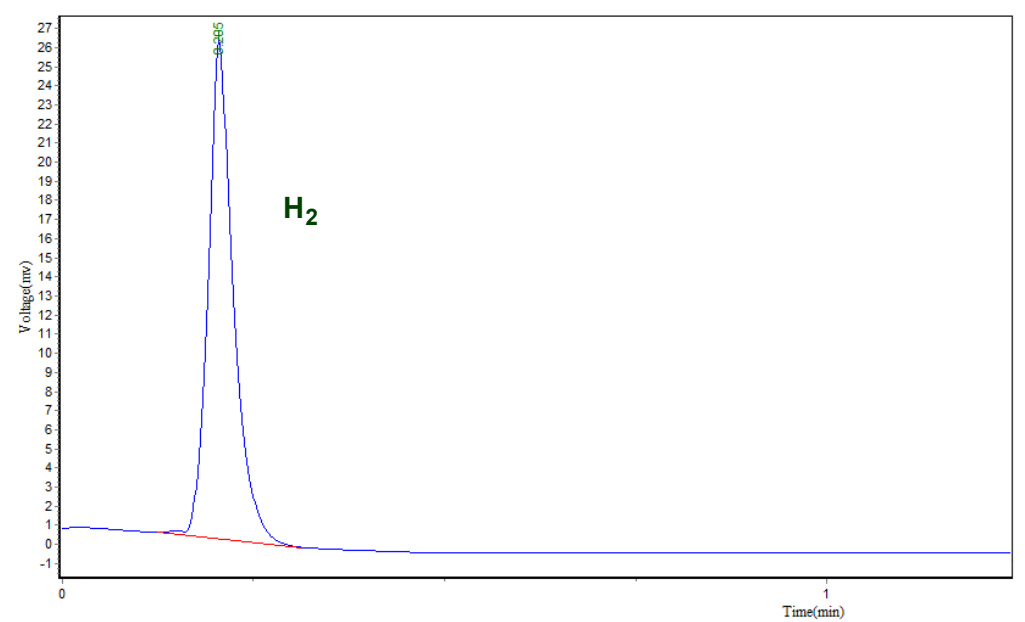


[1.5] Copies of ${ }^{1} \mathrm{H}$ NMR and ${ }^{13} \mathrm{C}$ NMR Spectra for selected compounds

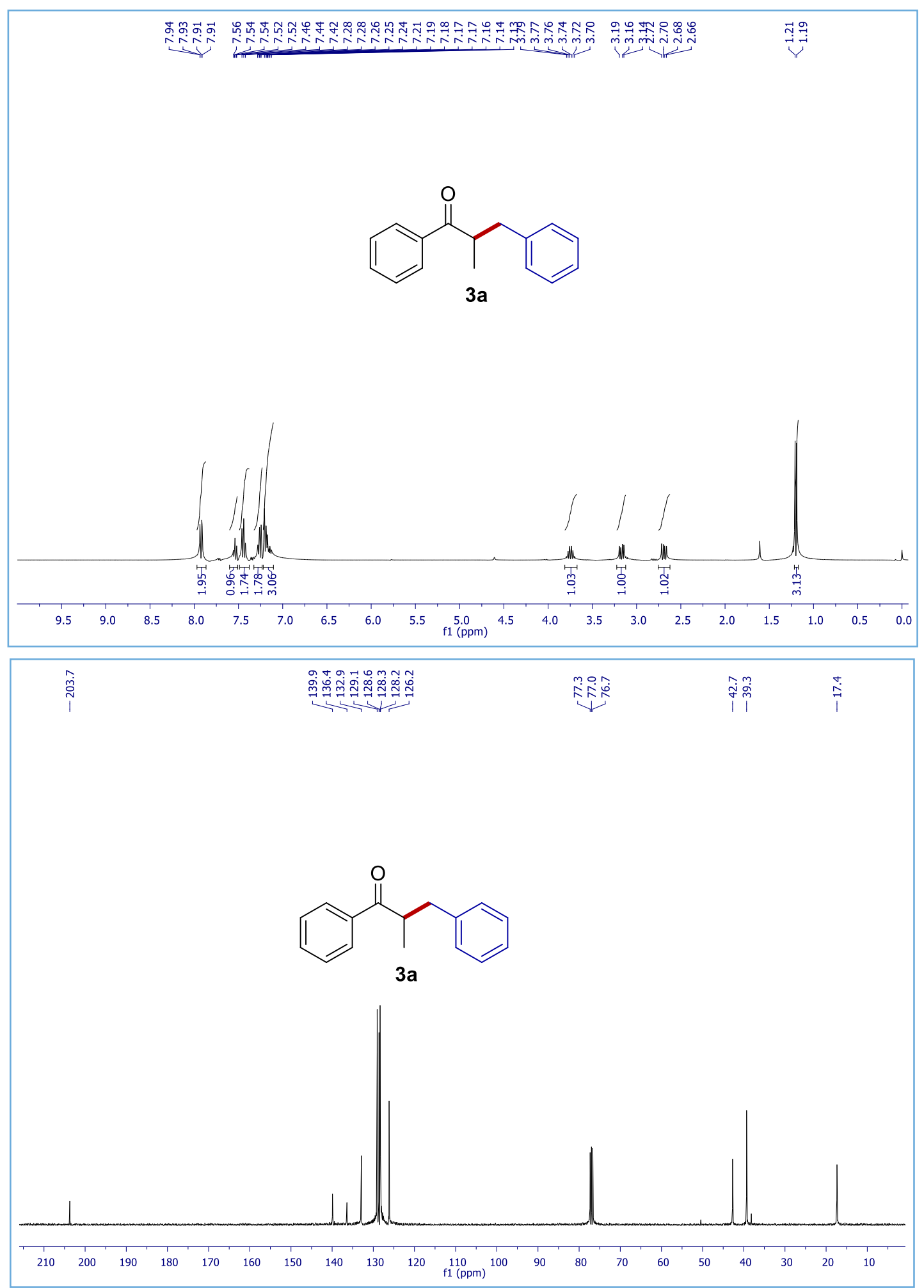

S18 


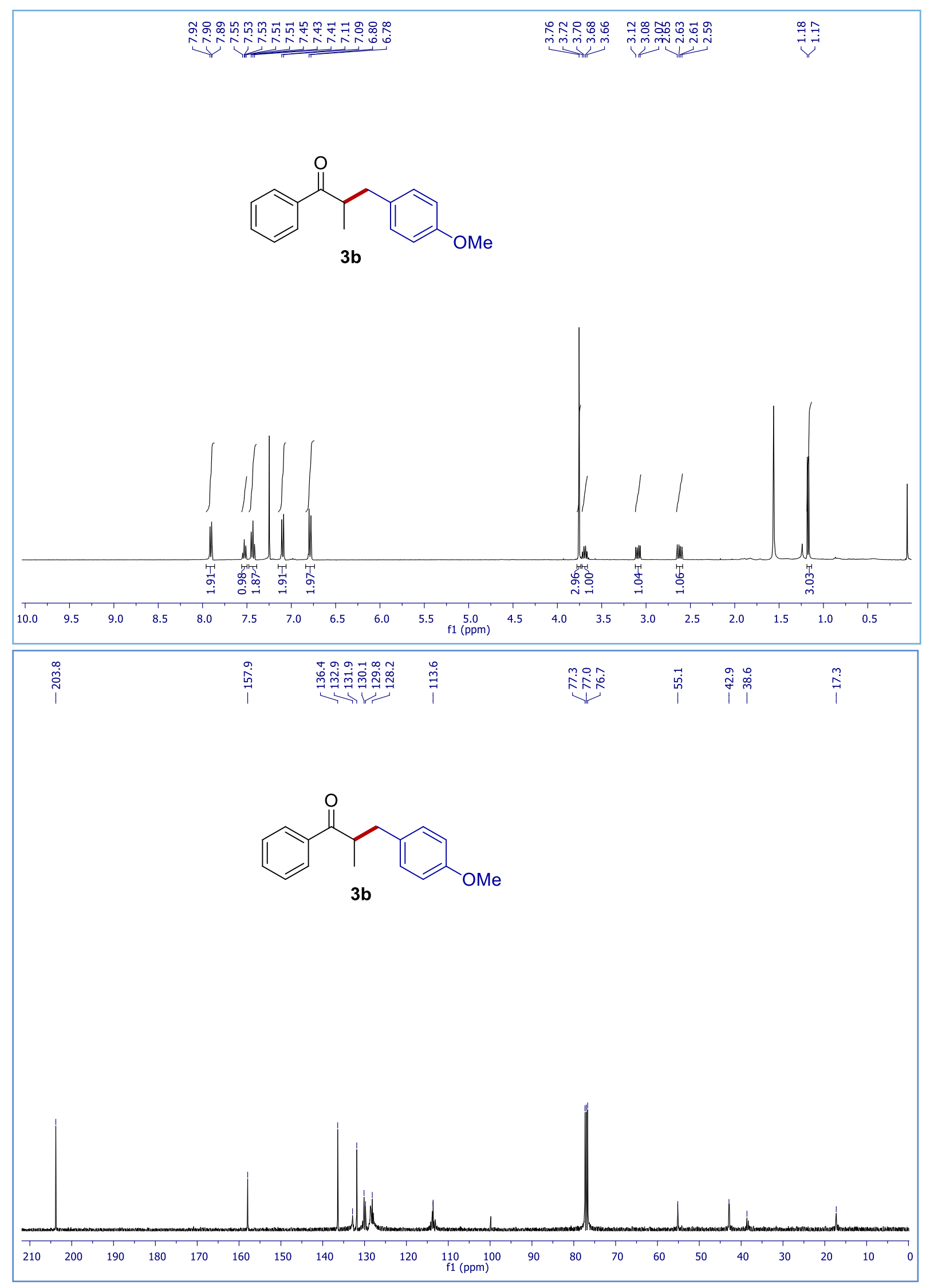

S19 


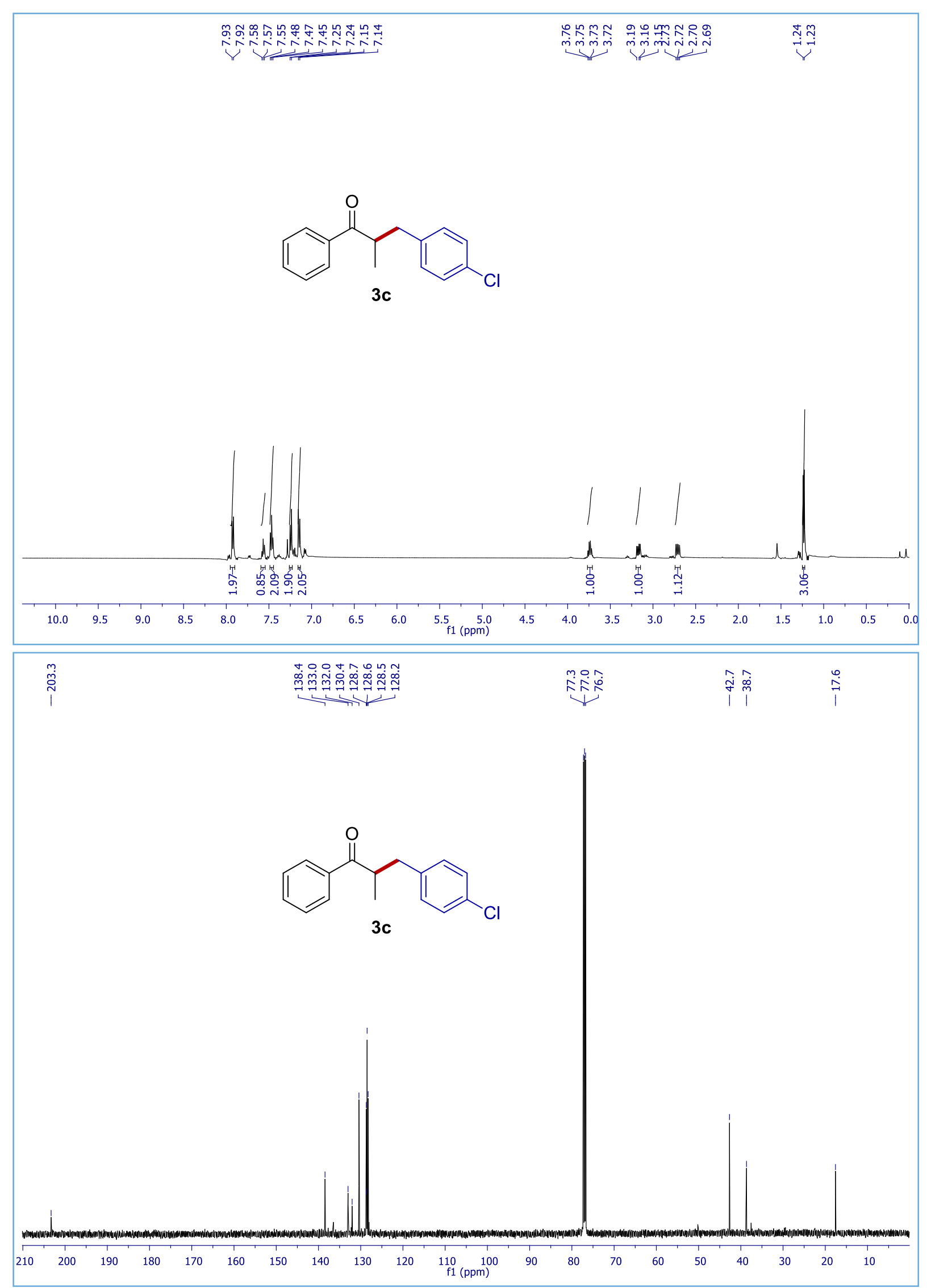

S20 


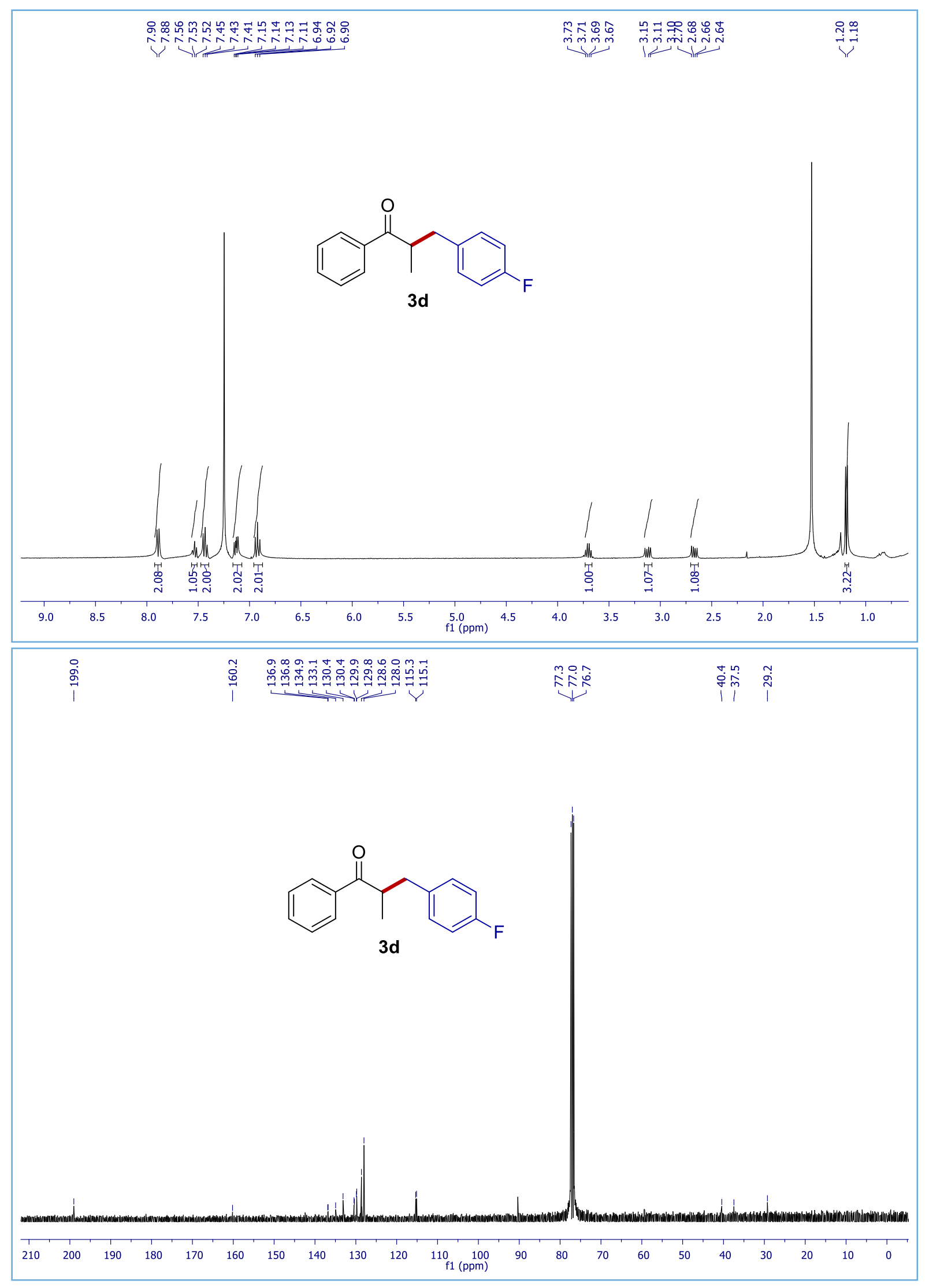

S21 


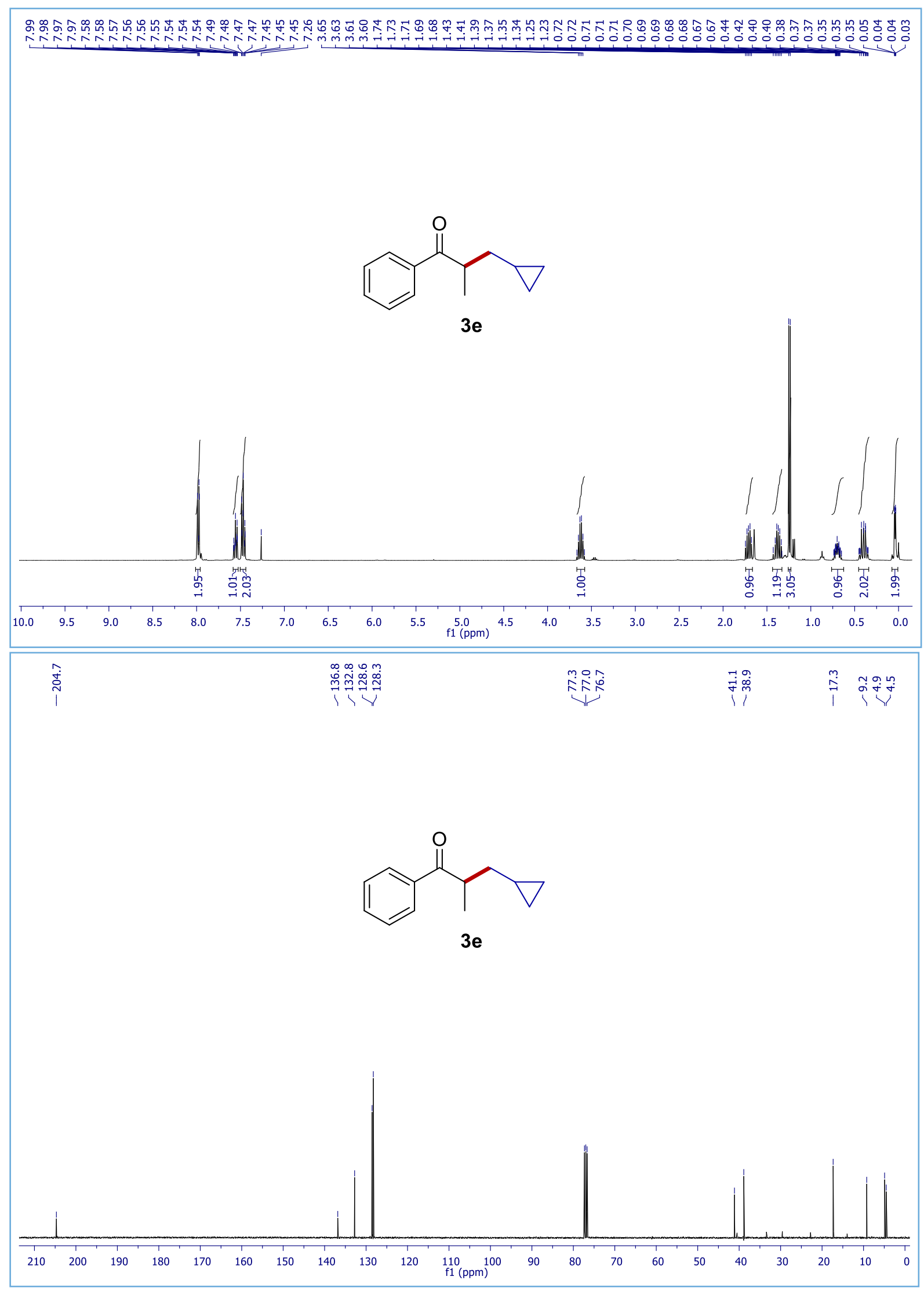

S22 


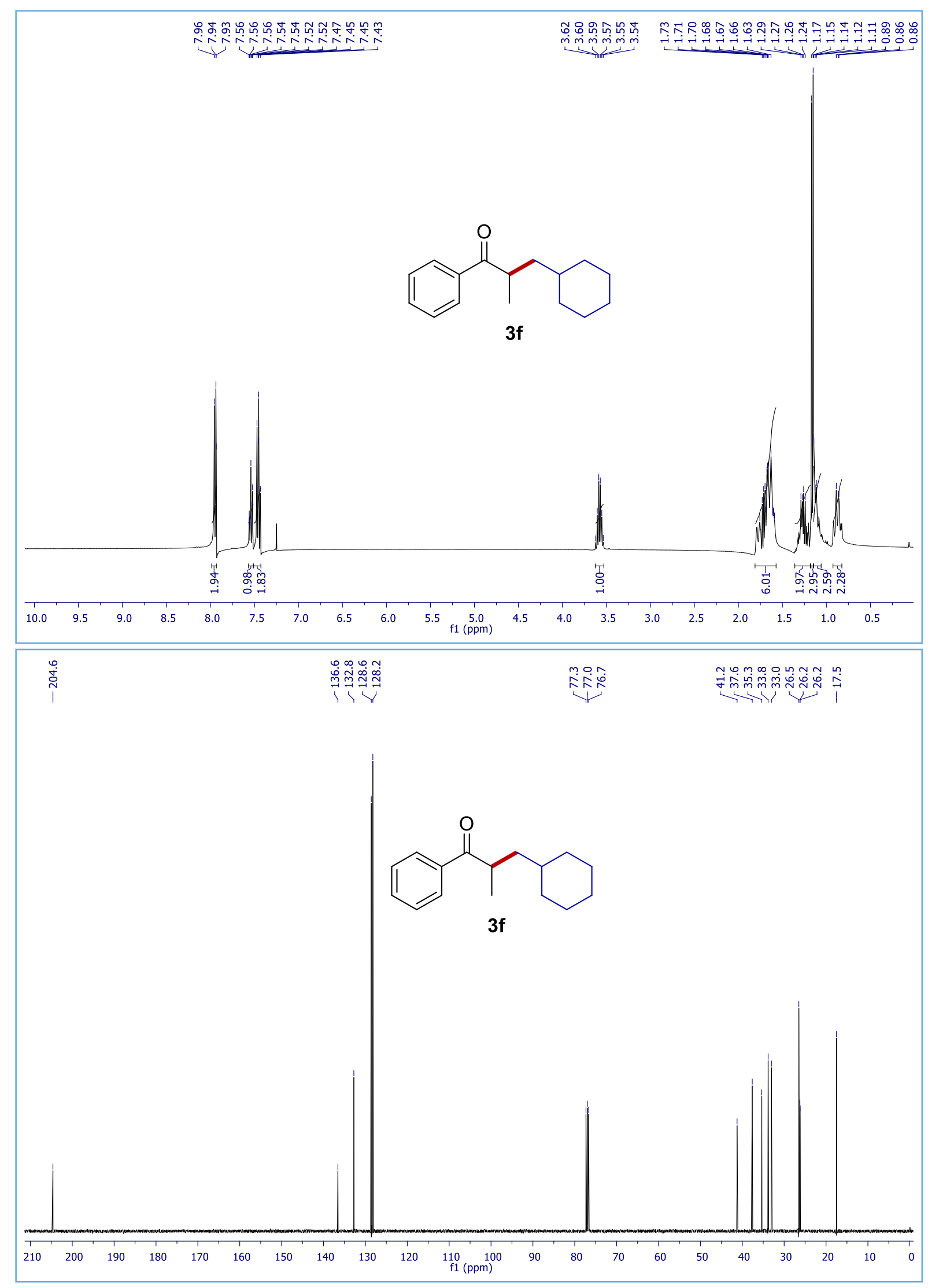




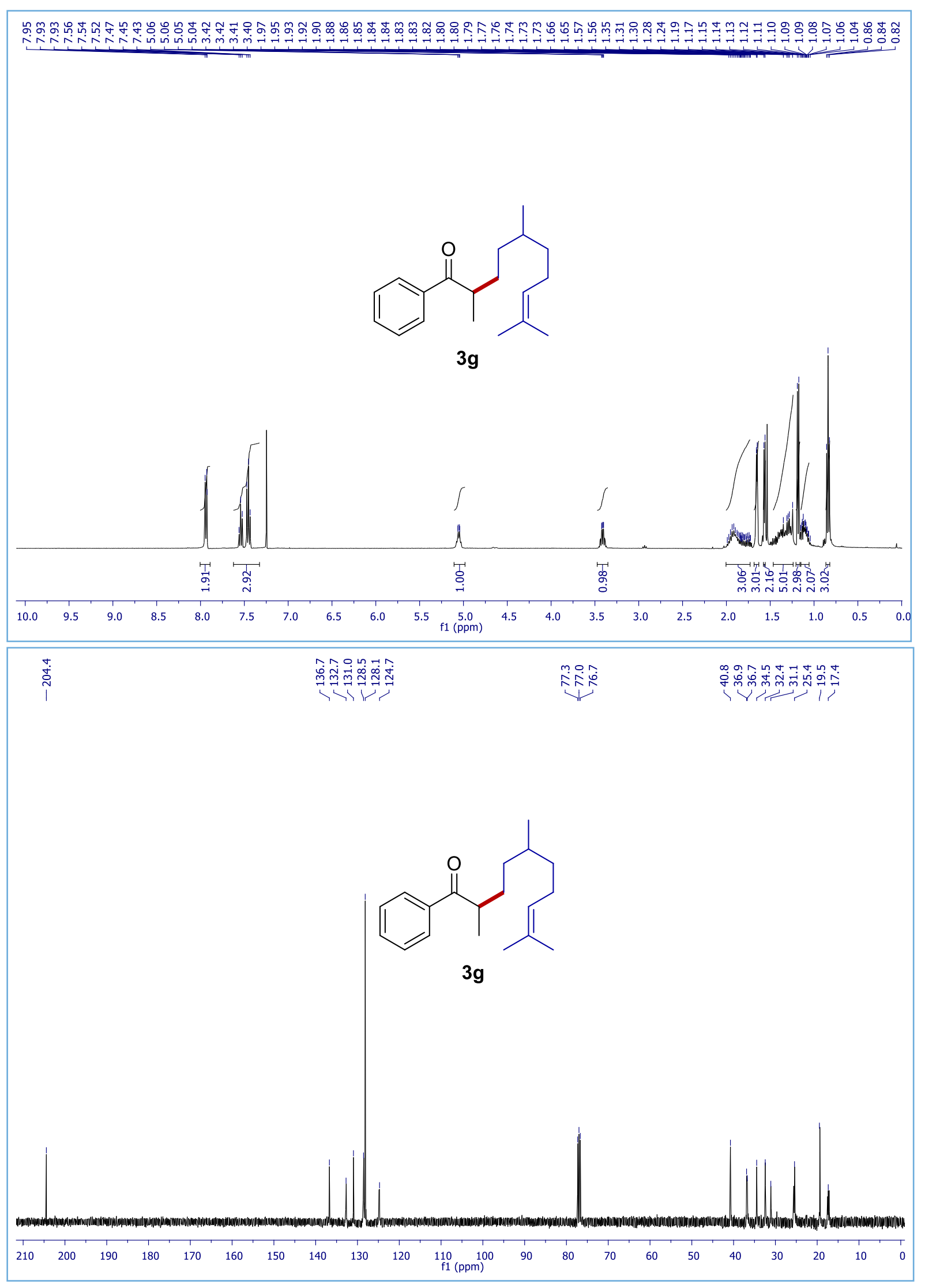



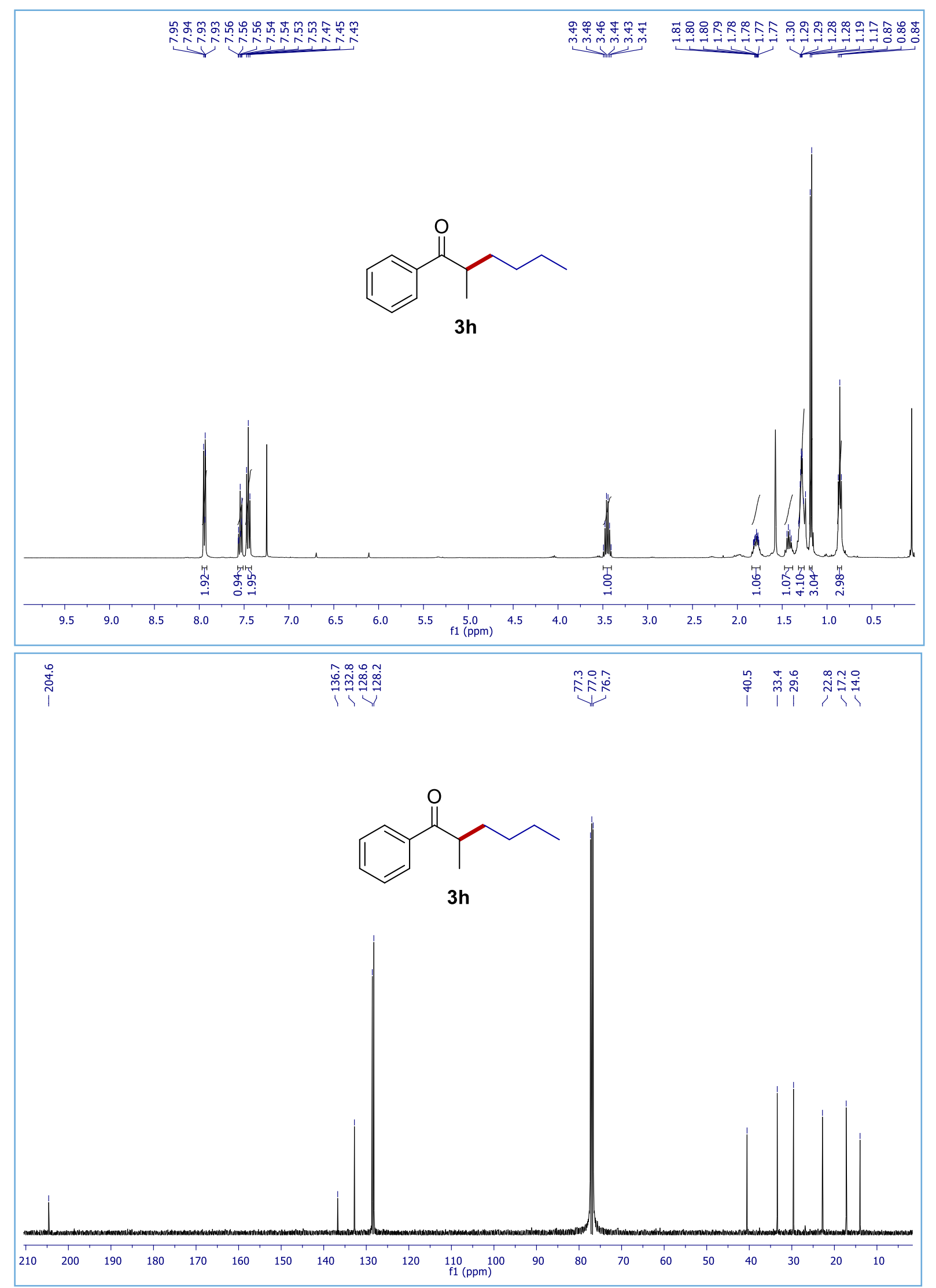


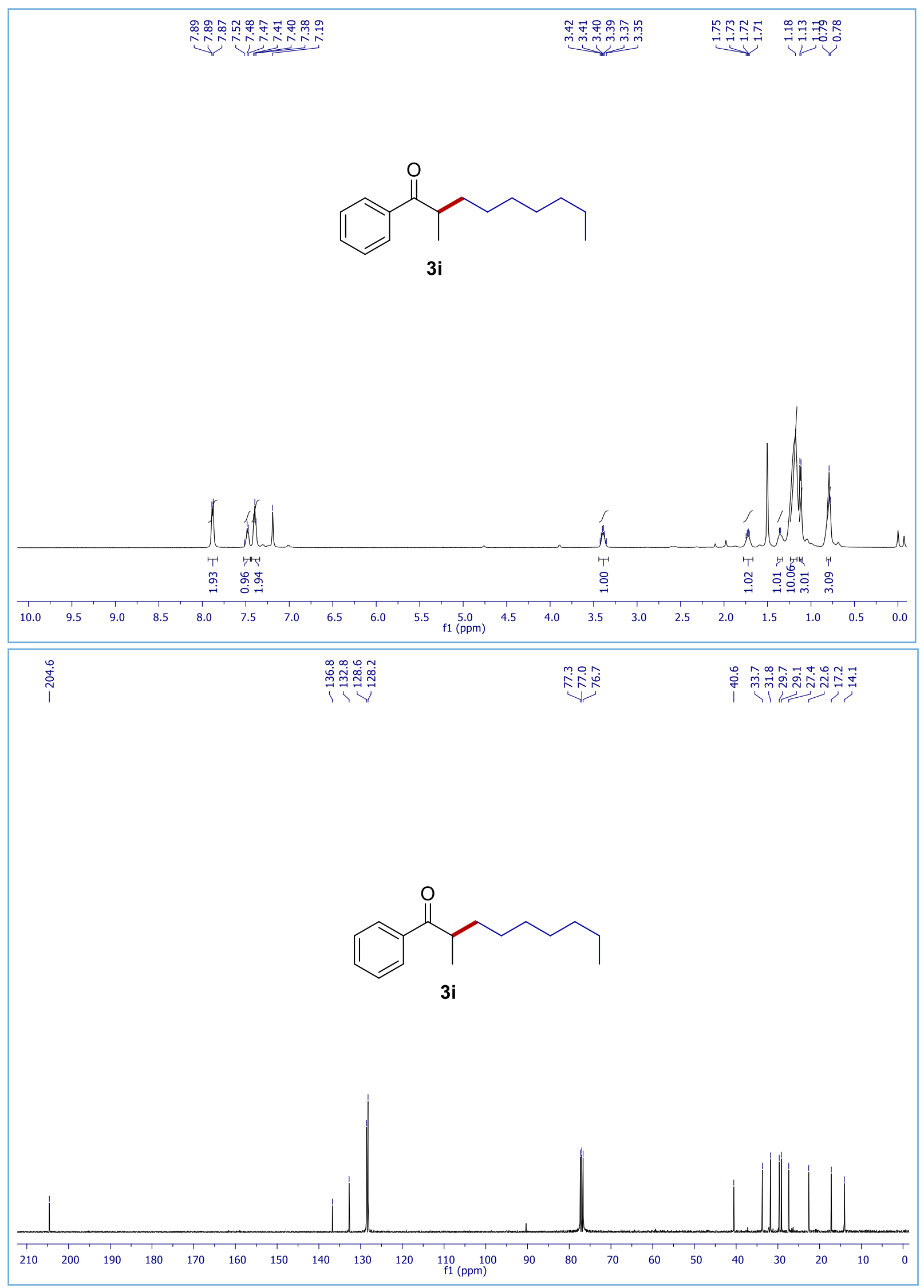



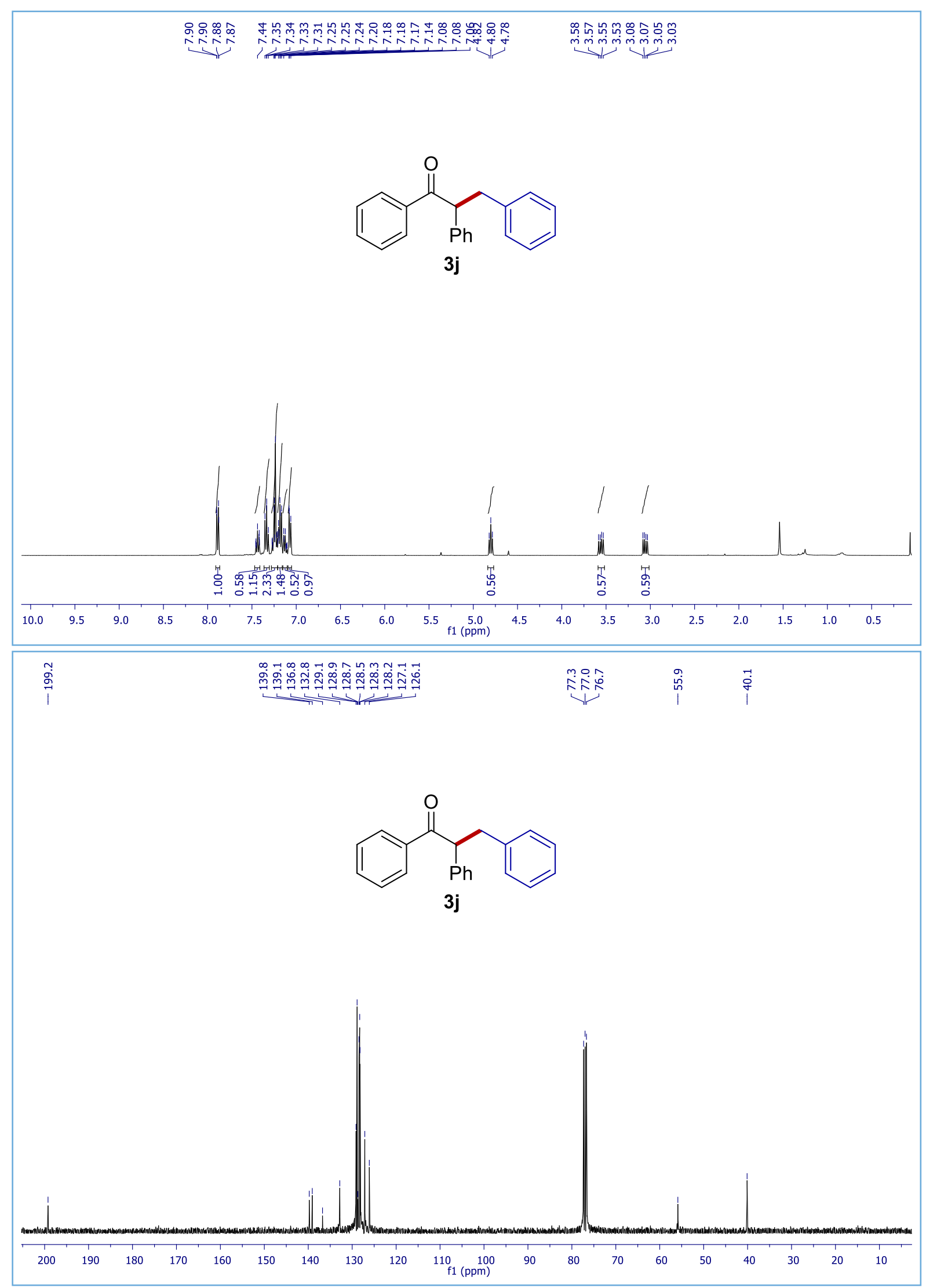

S27 


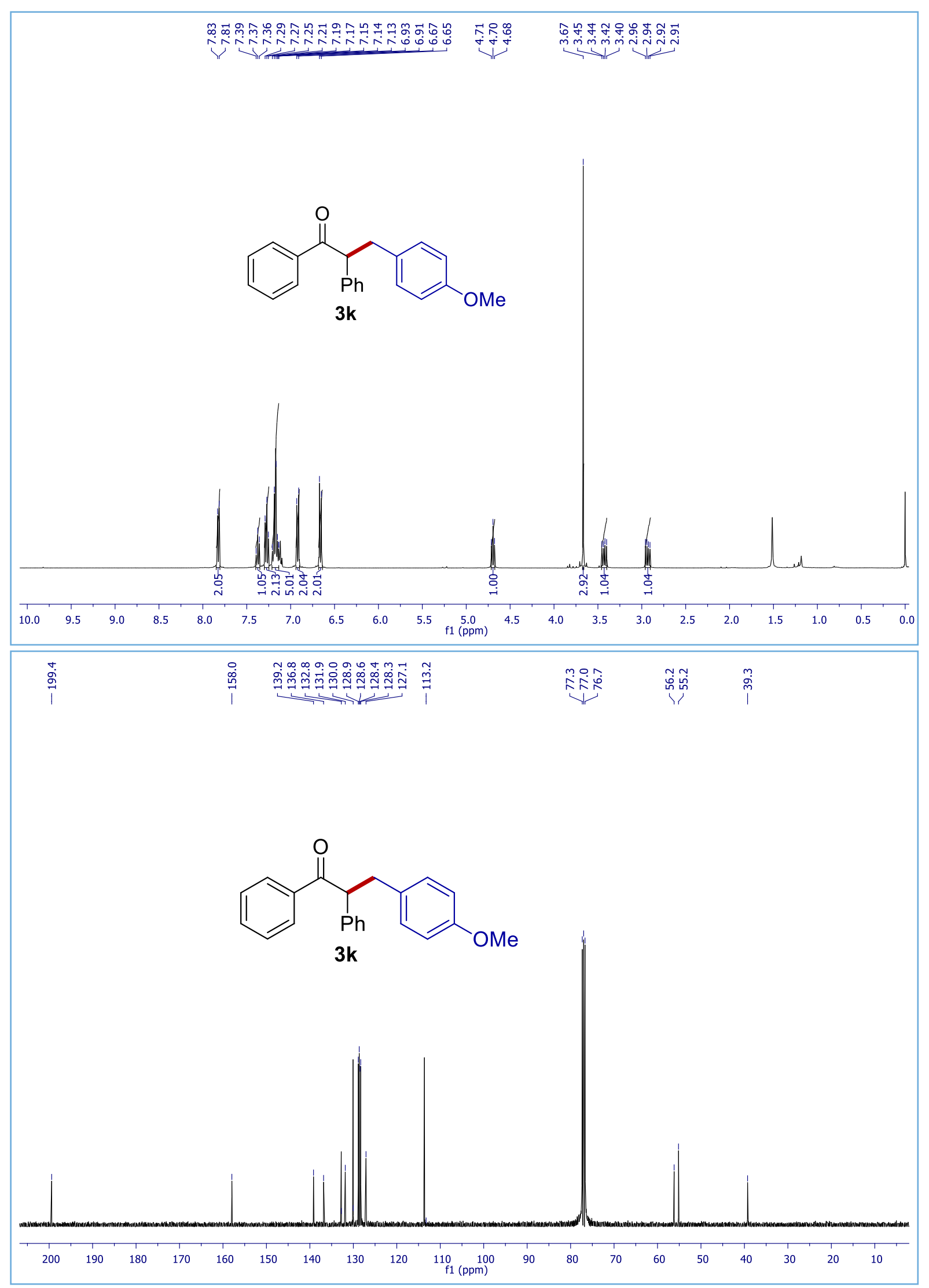

S28 


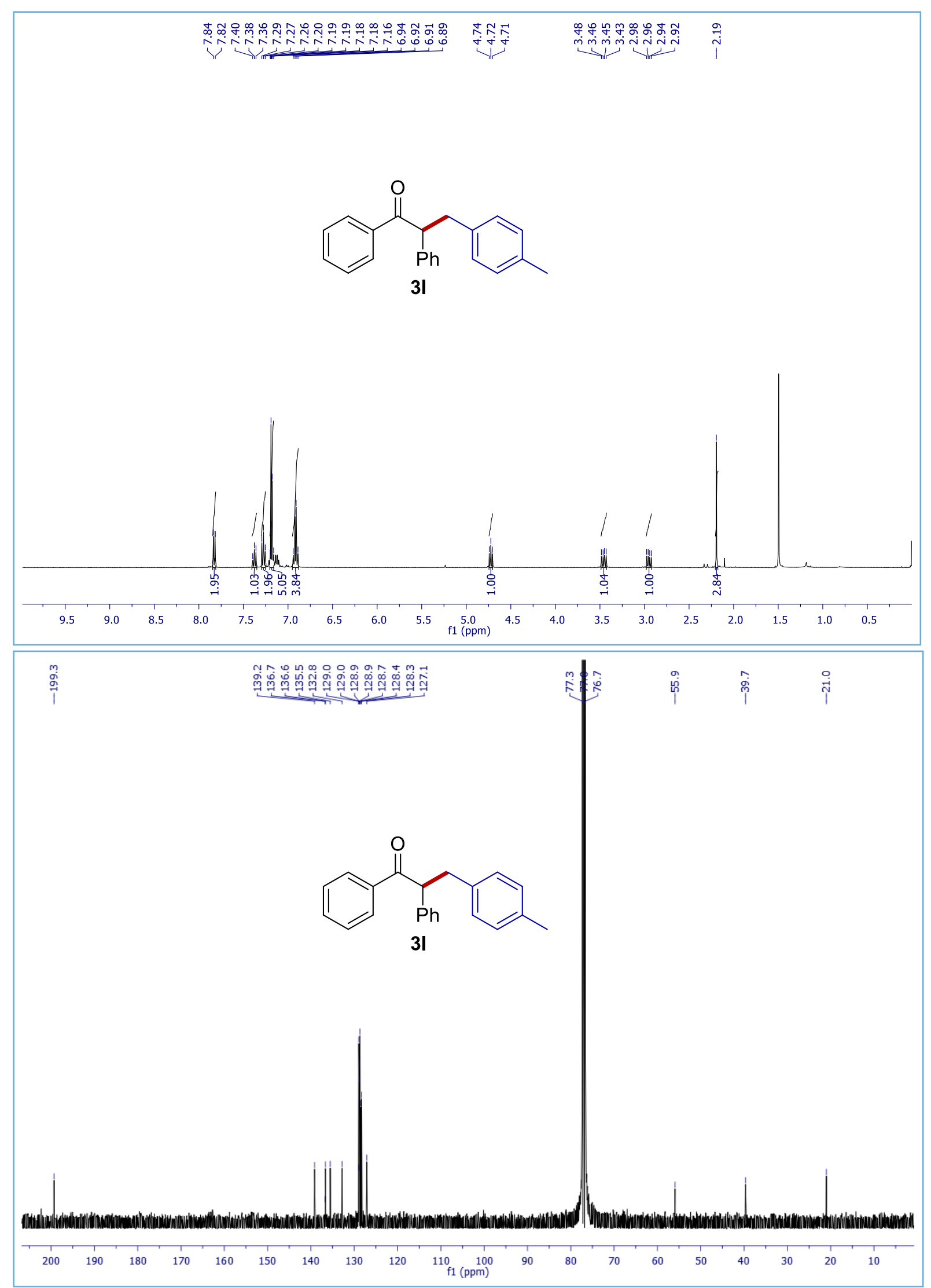

S29 


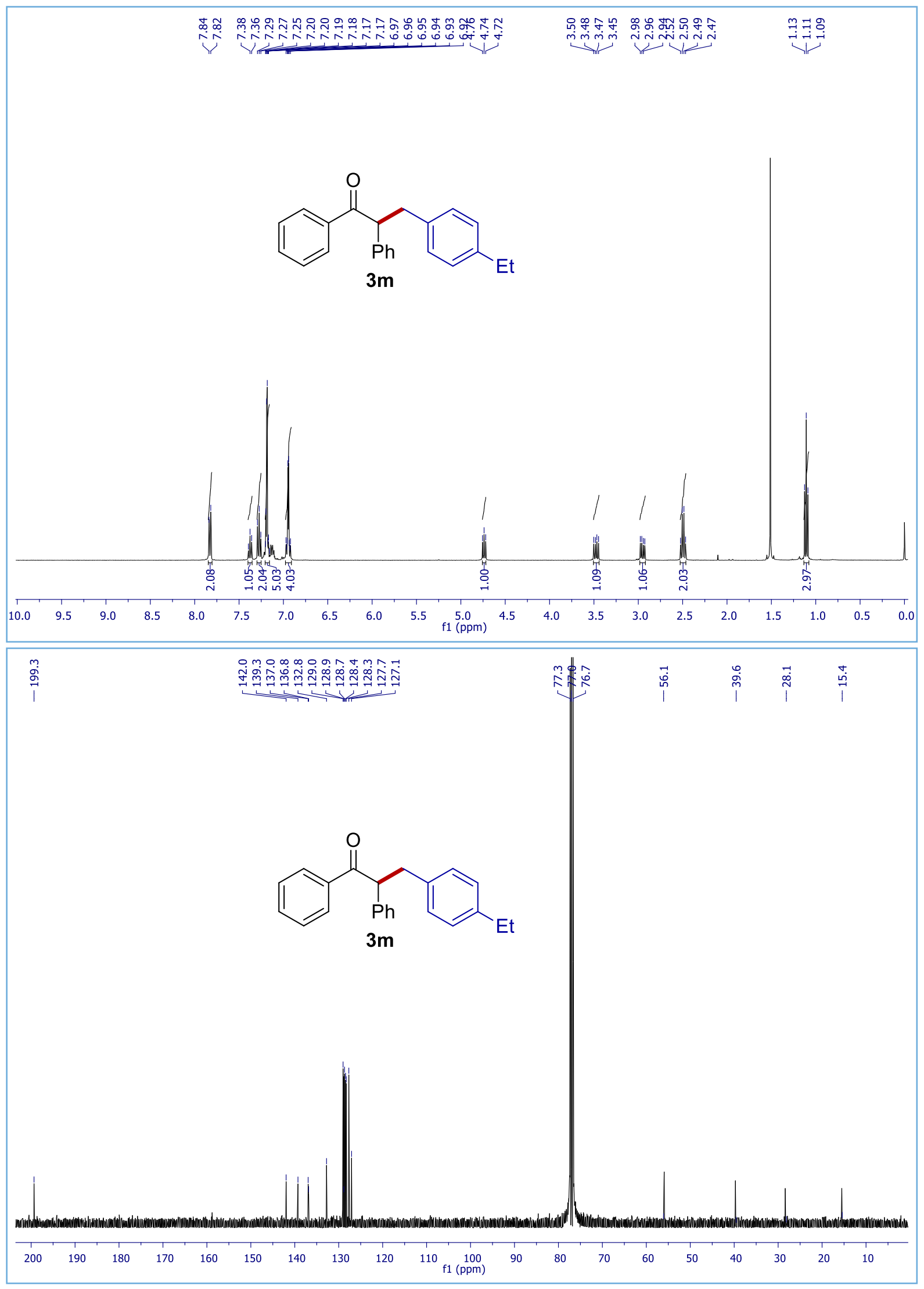



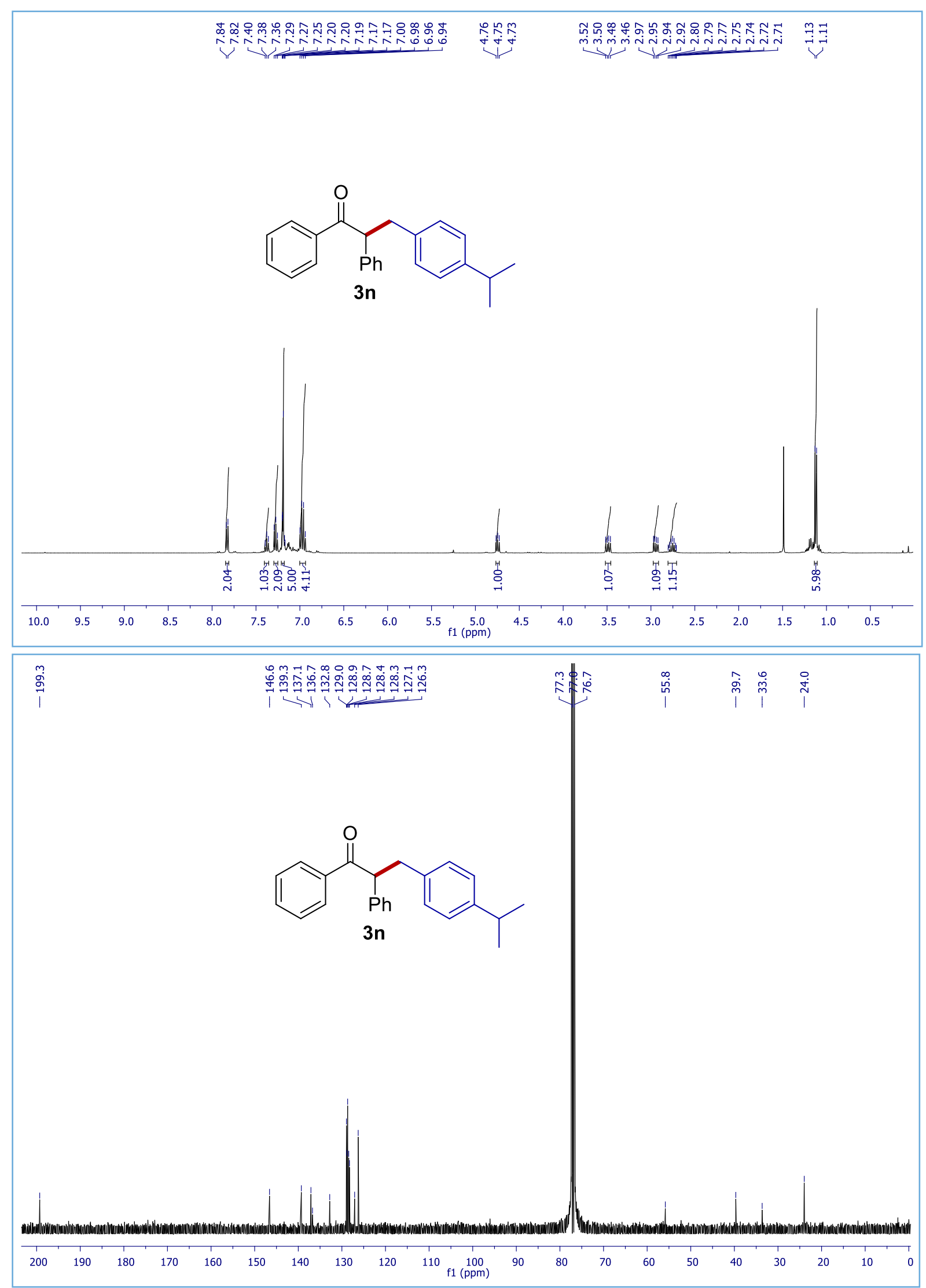

S31 


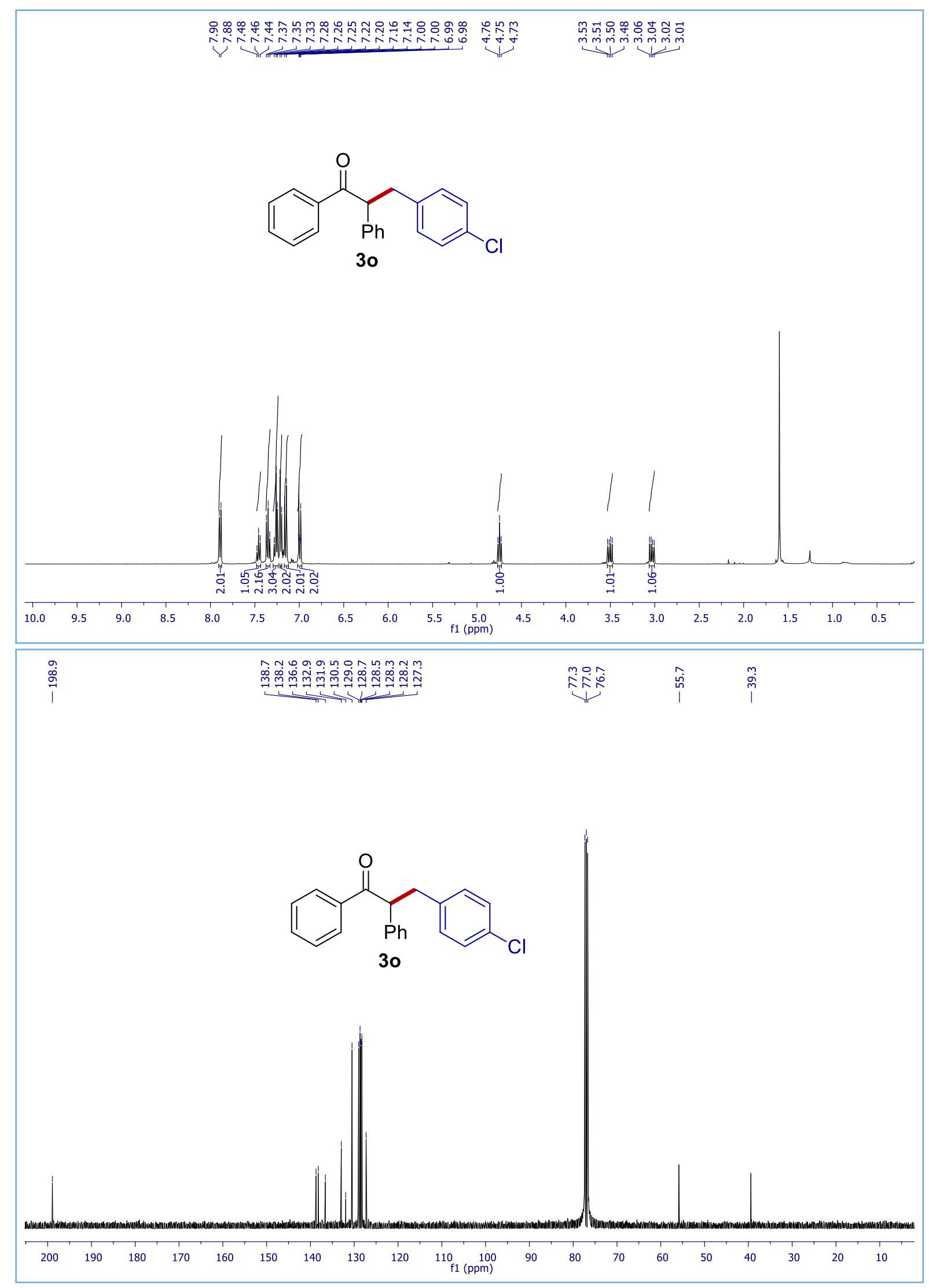




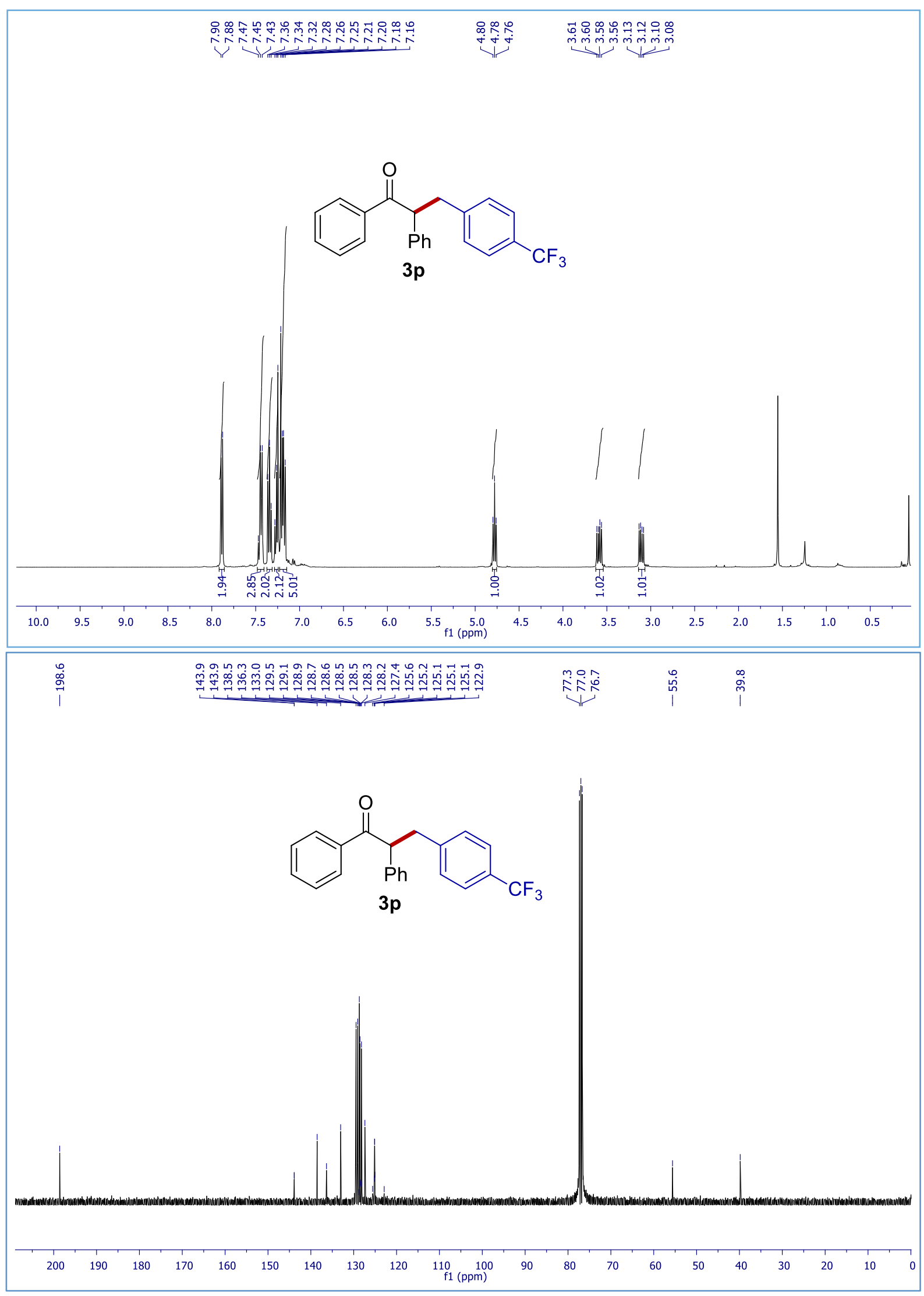



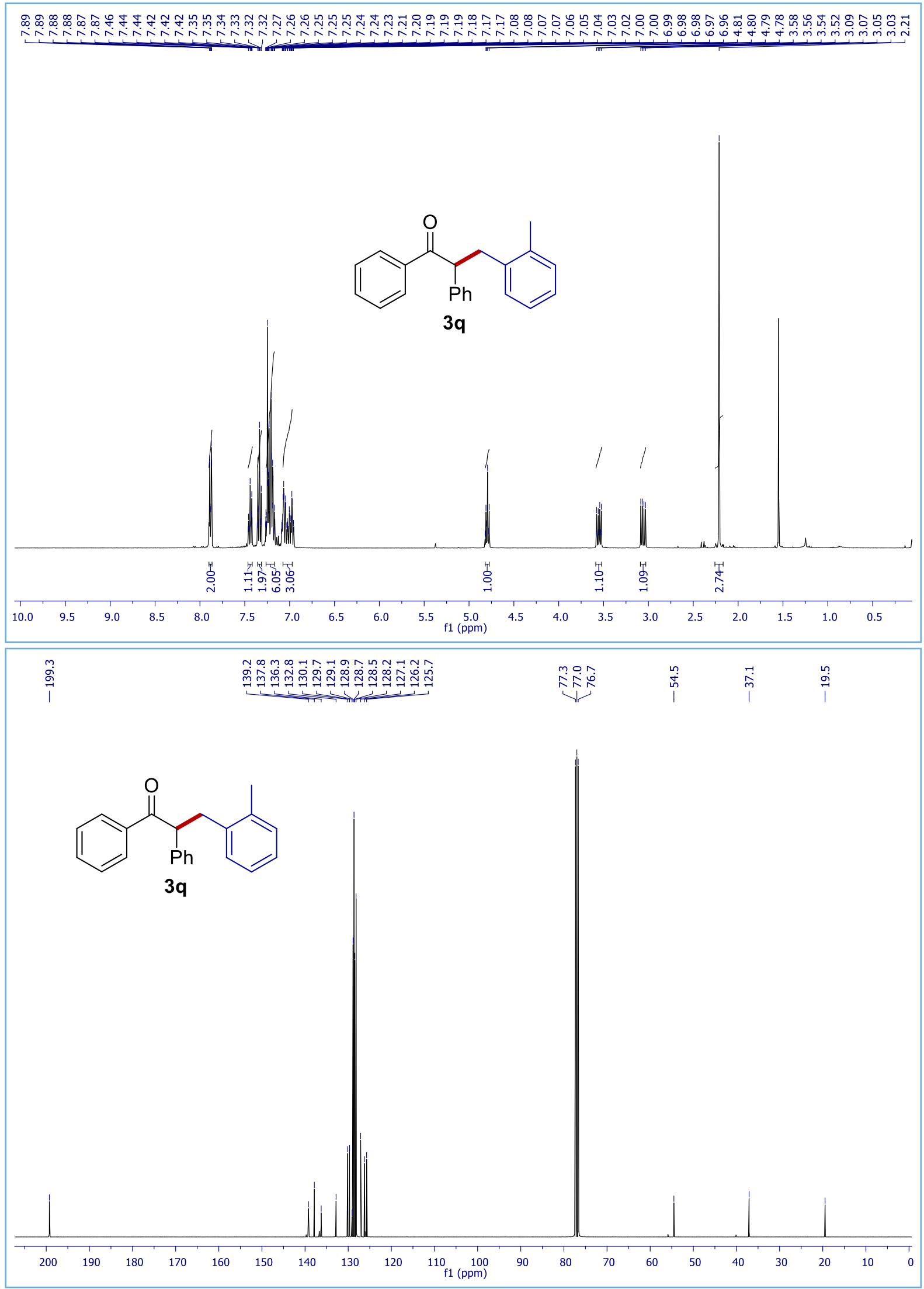


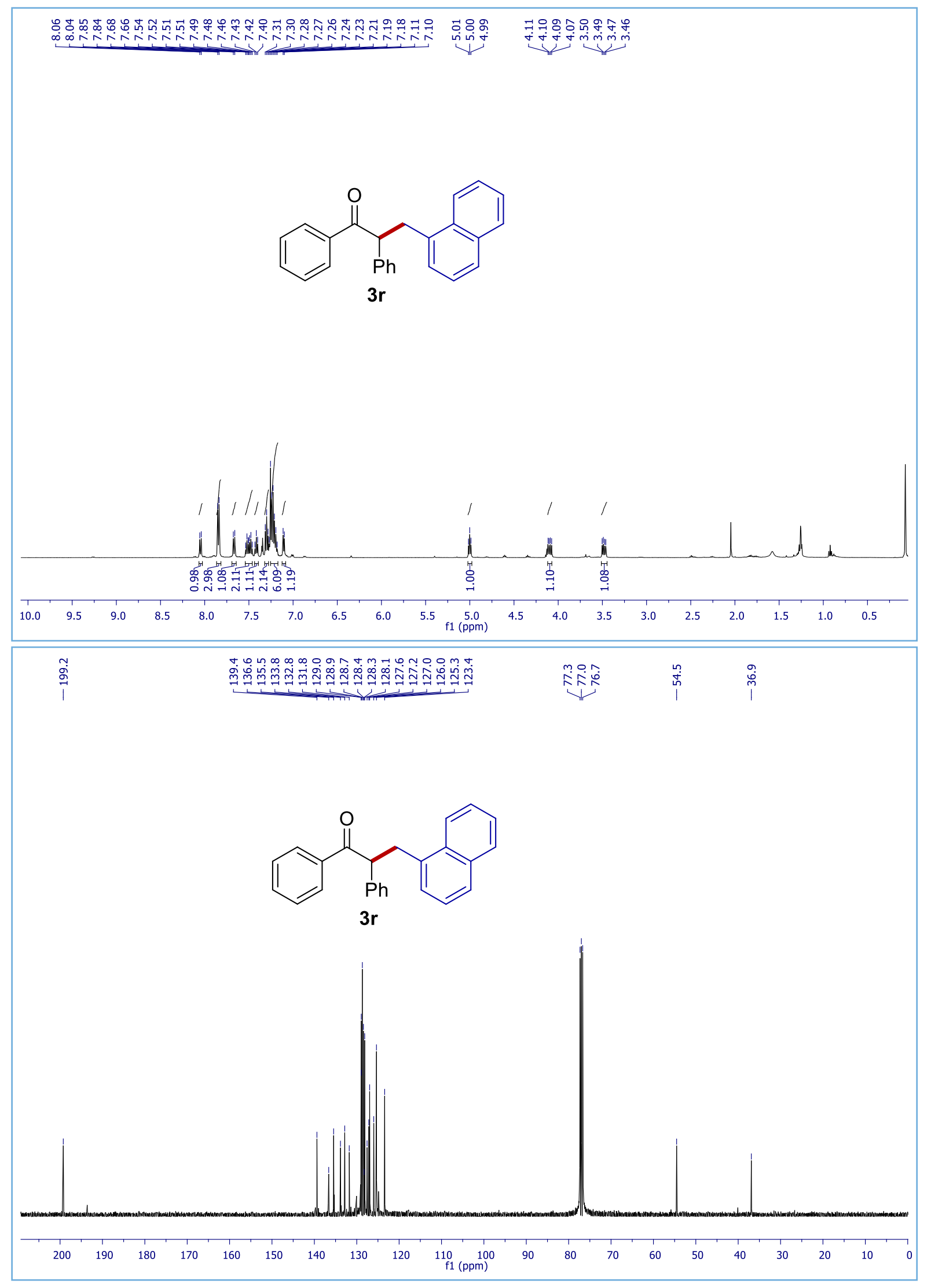

S35 

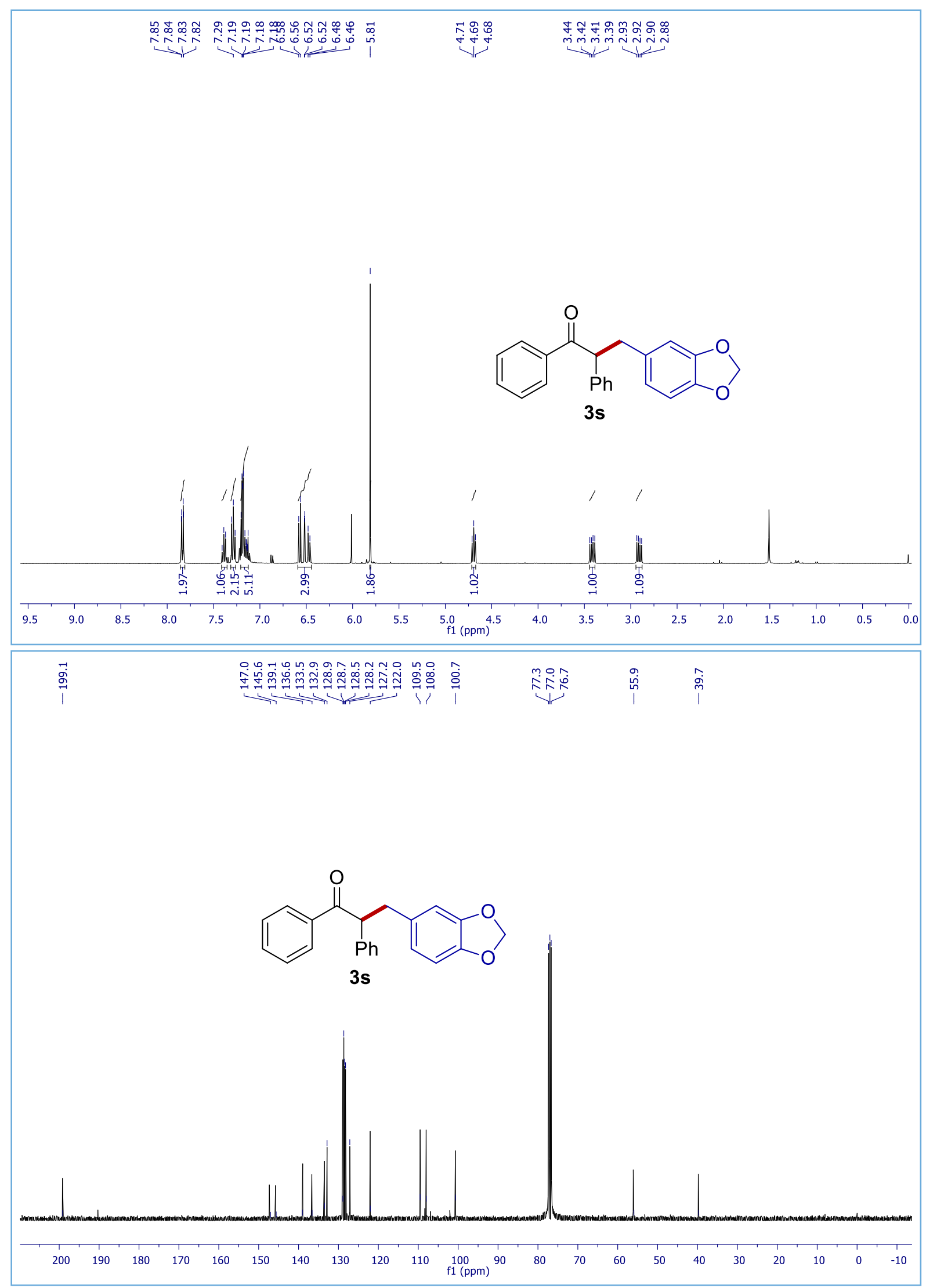

S36 


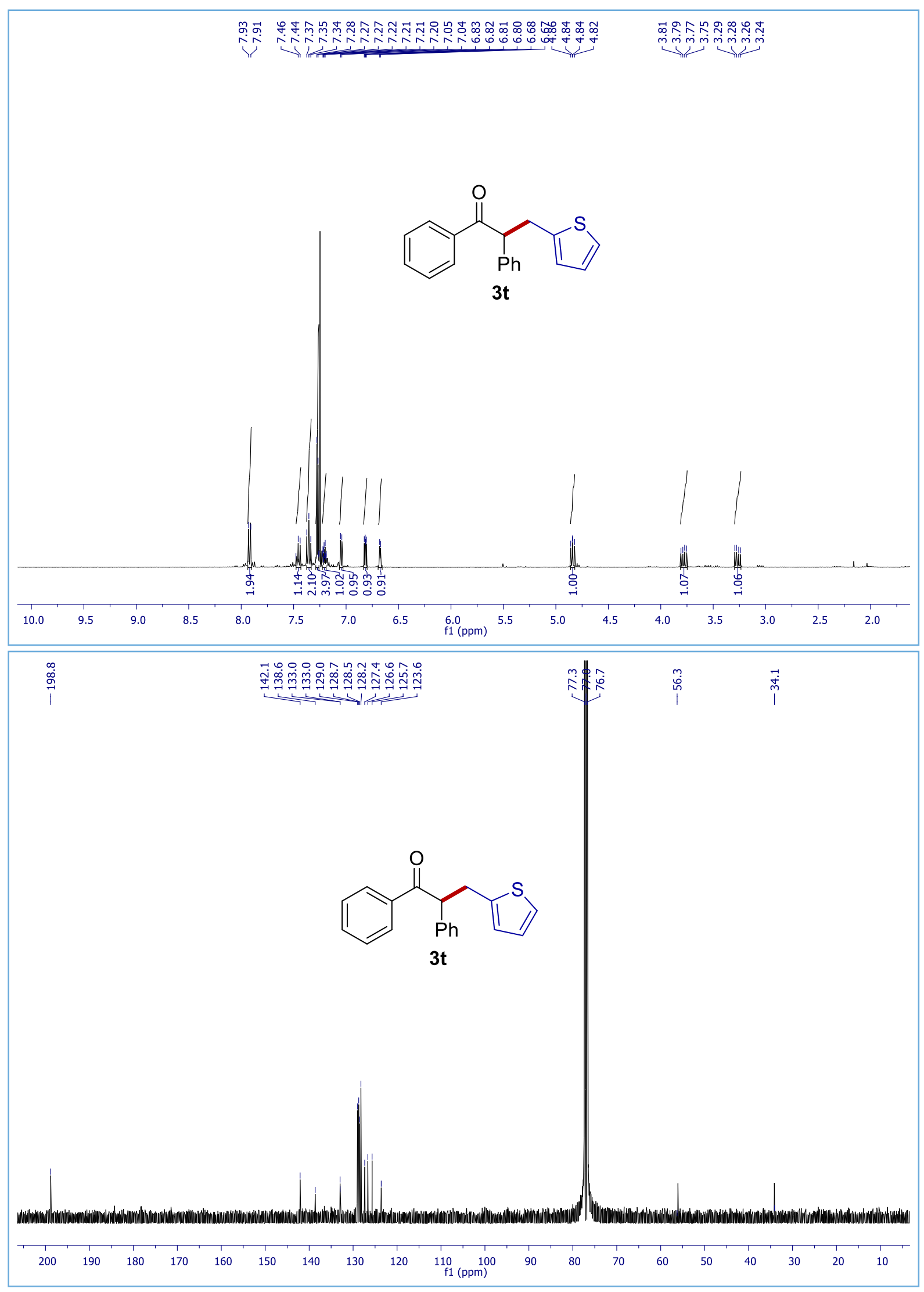




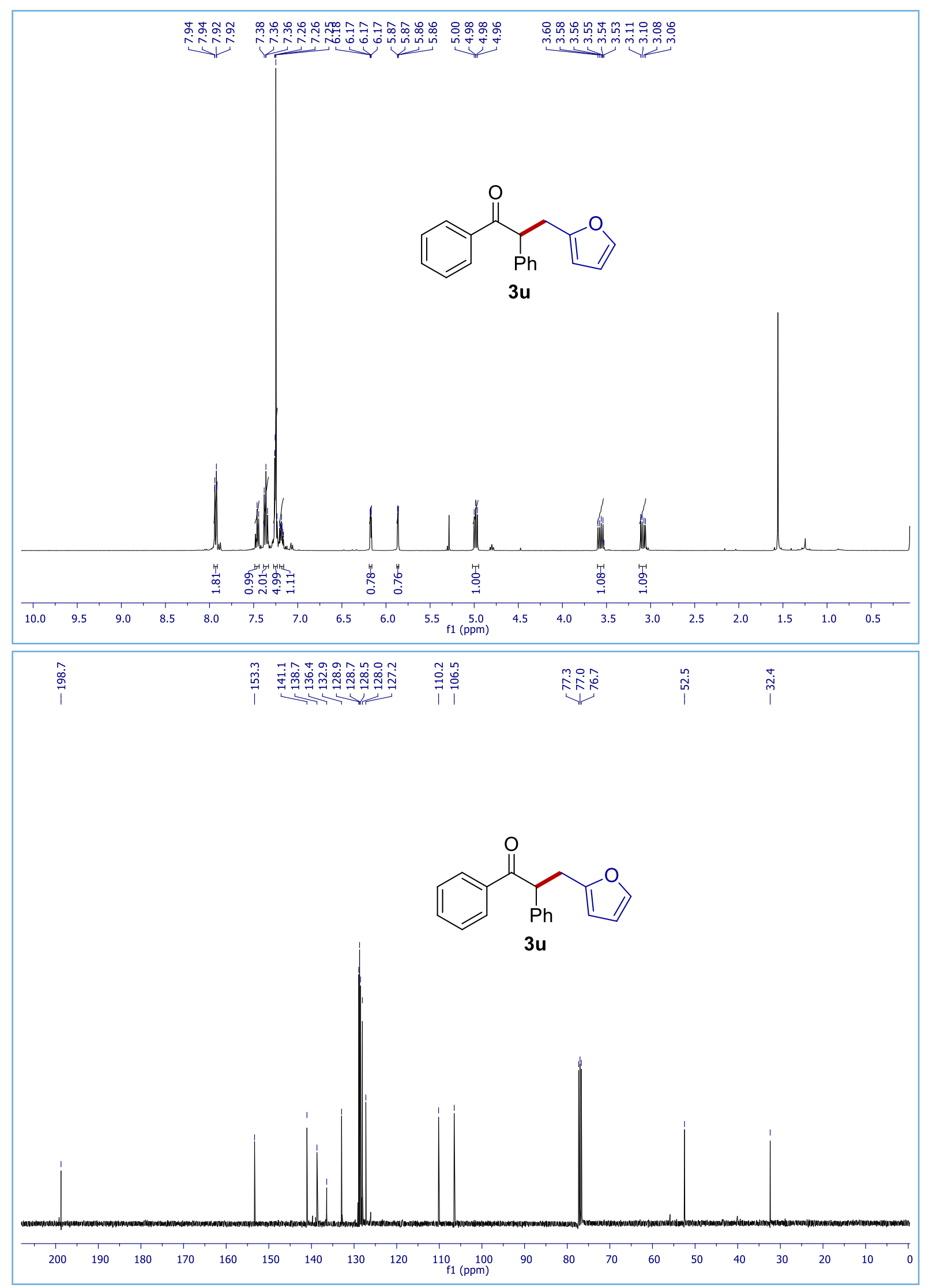

S38 


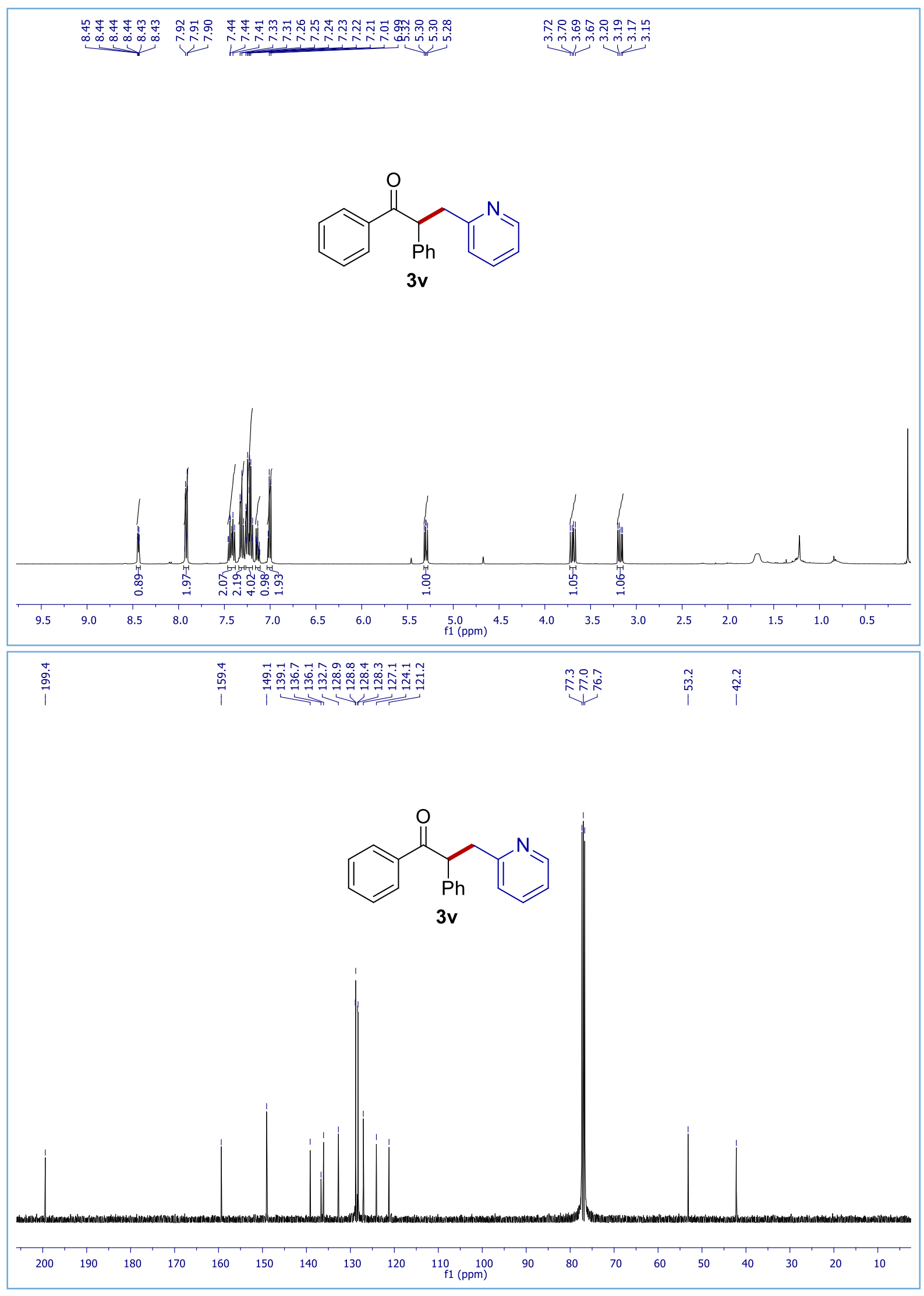




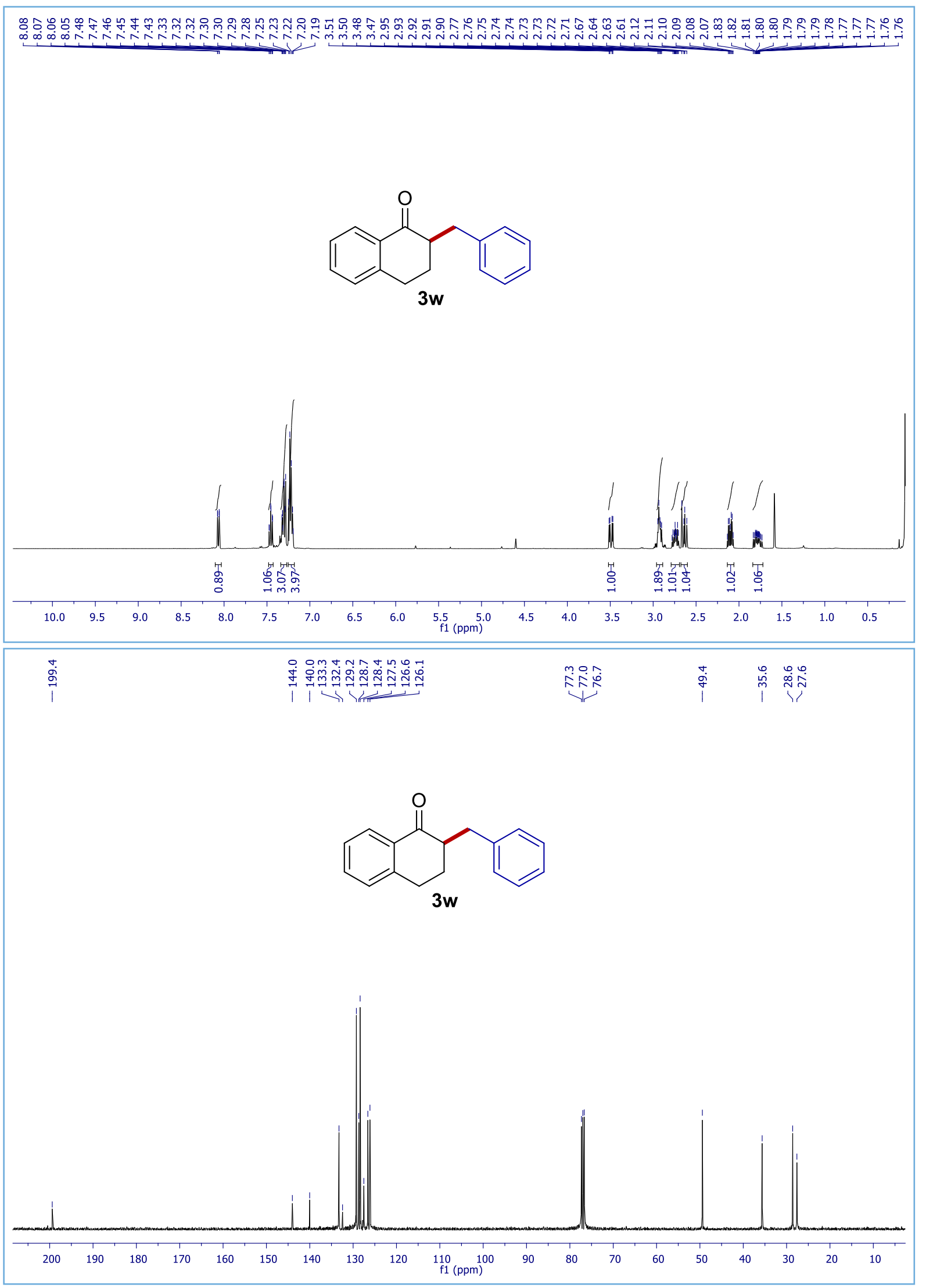

S40 


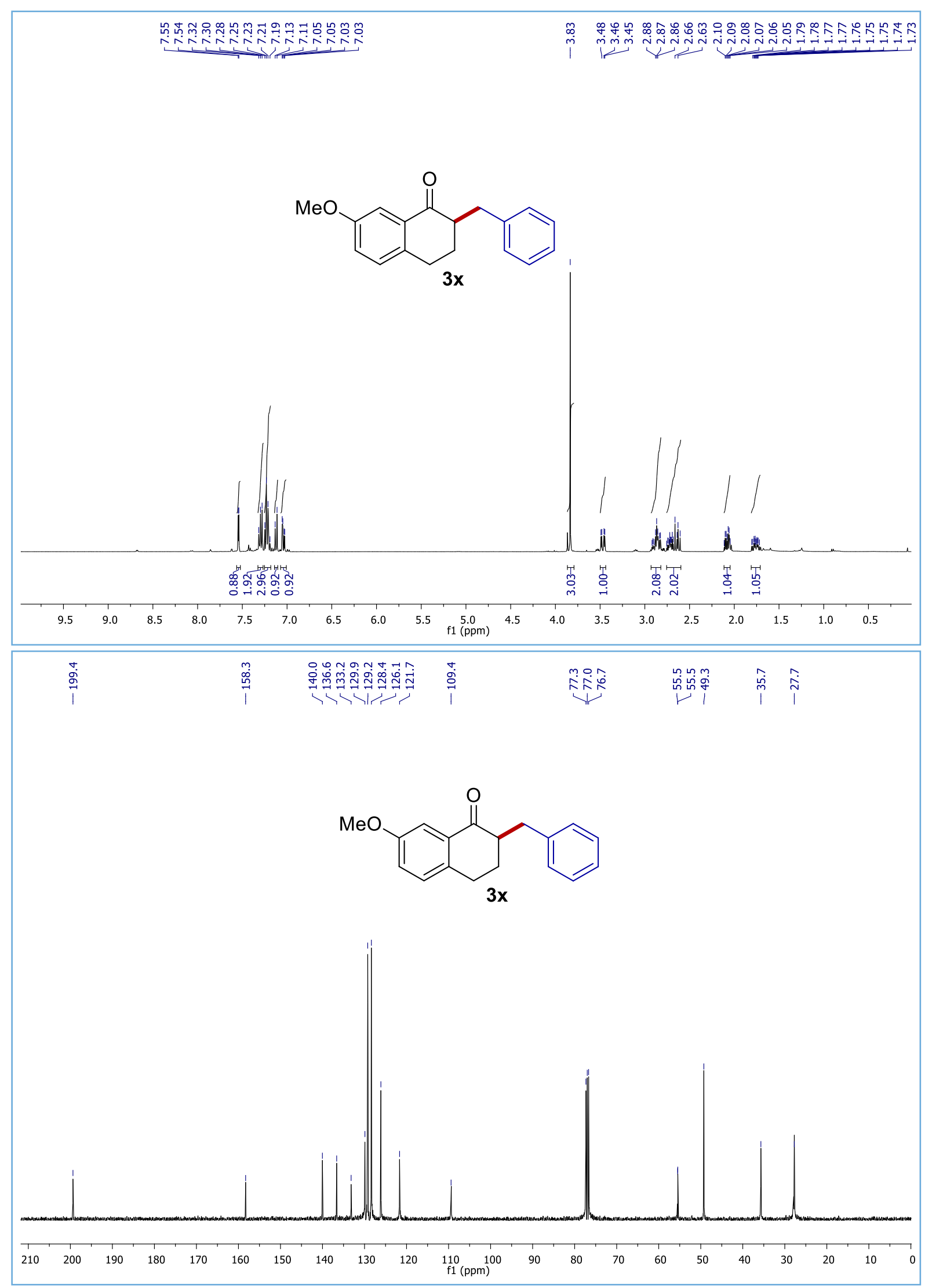

S41 


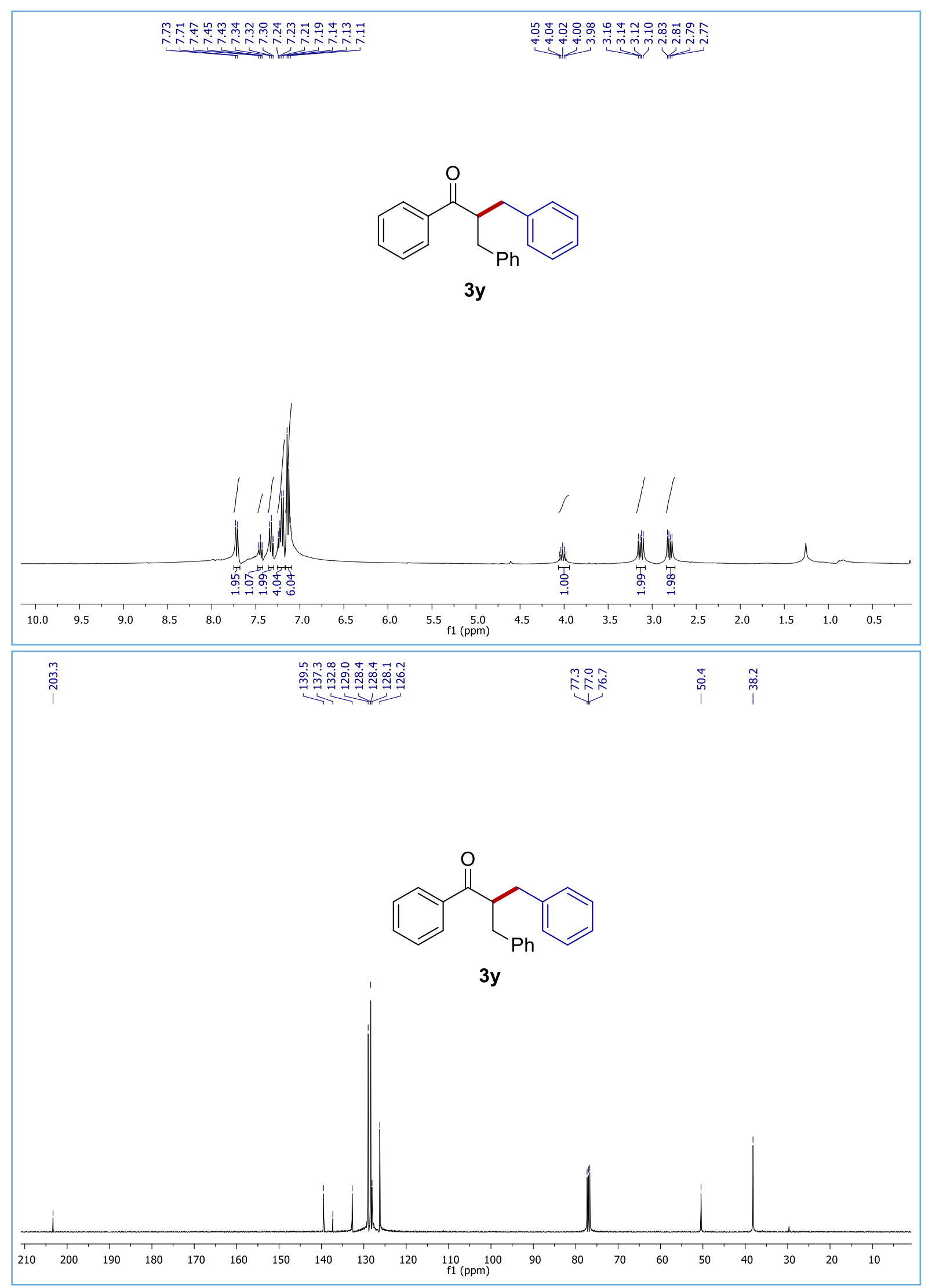

S42 


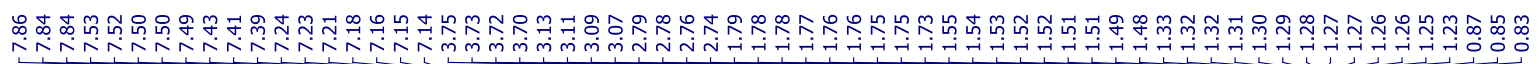
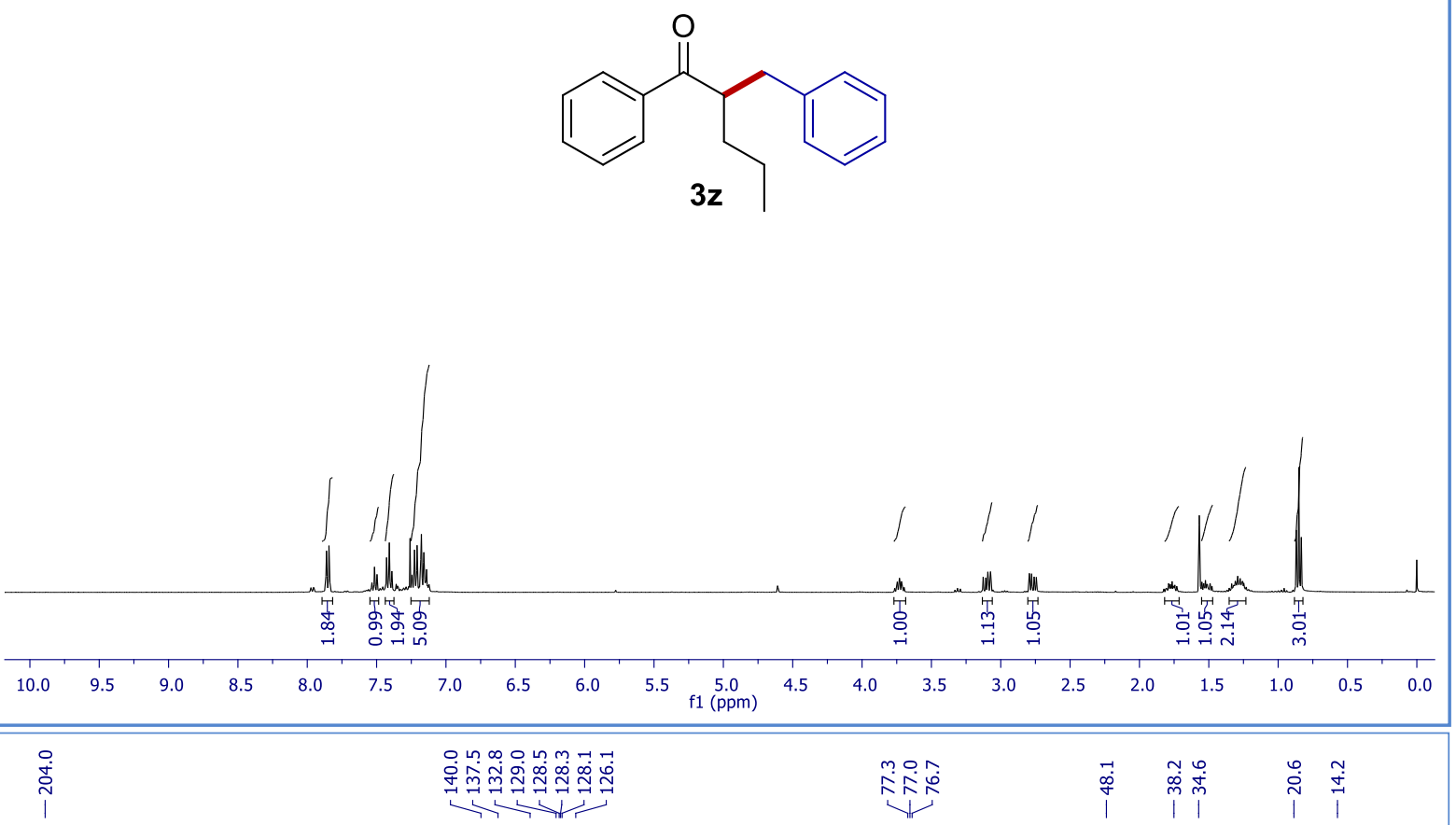

象

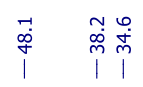

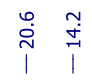
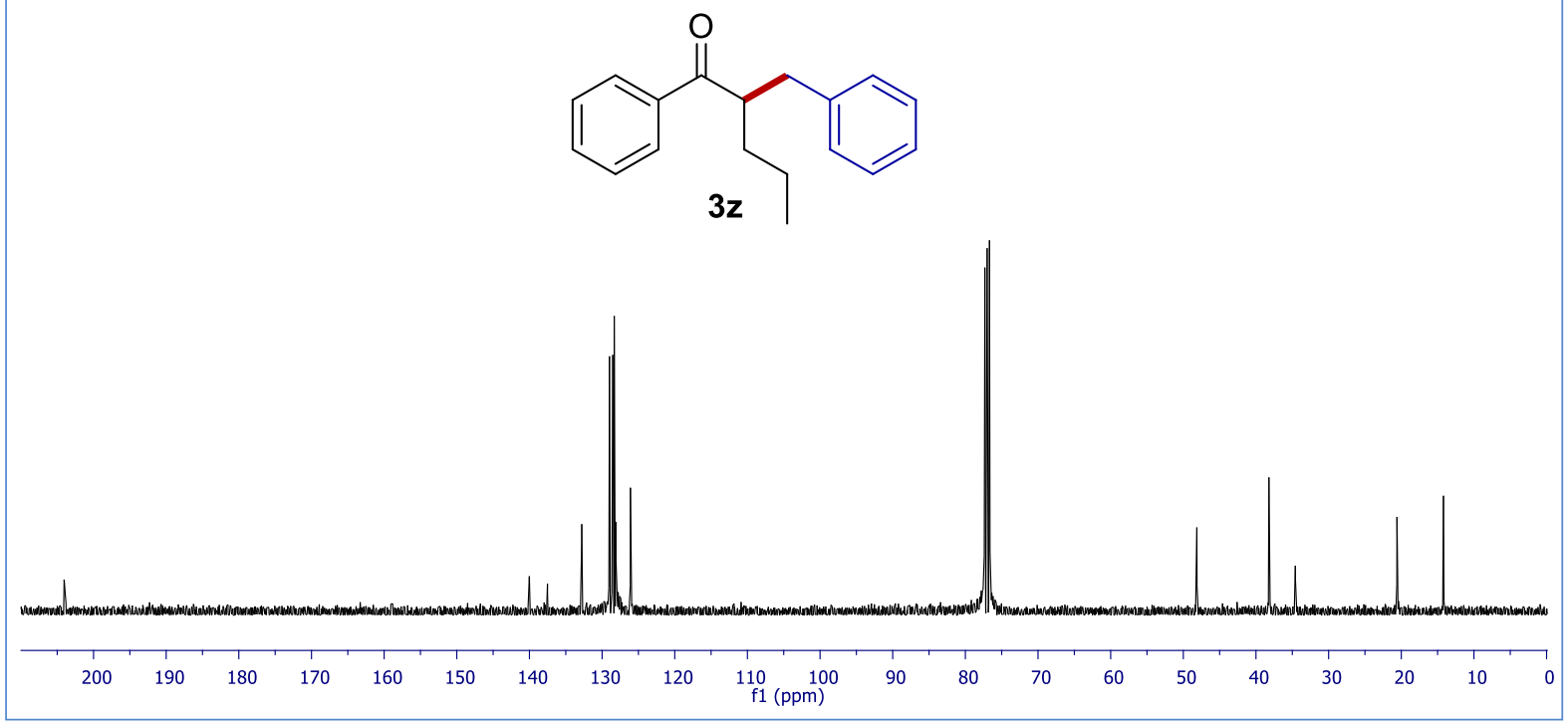

S43 


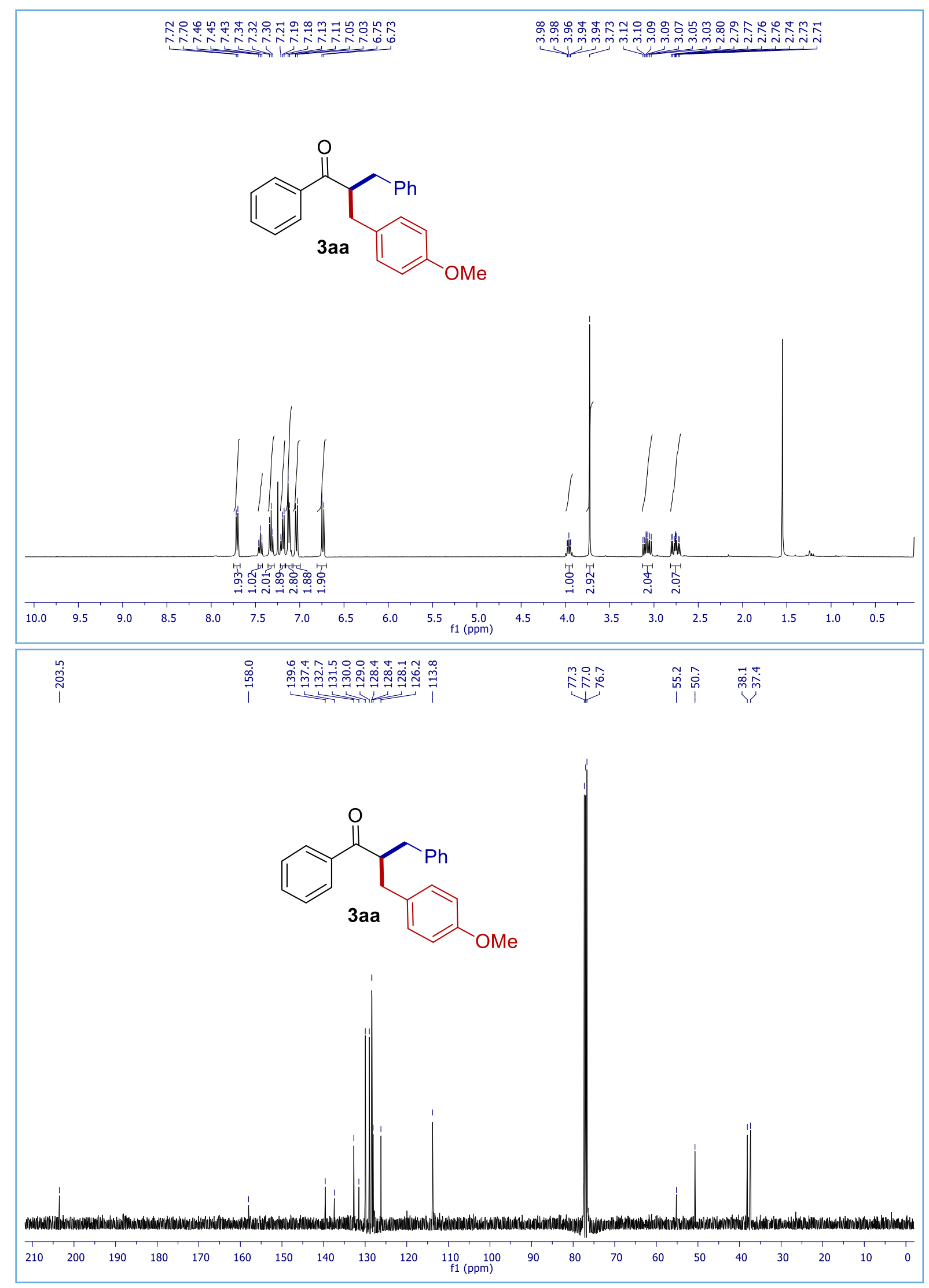




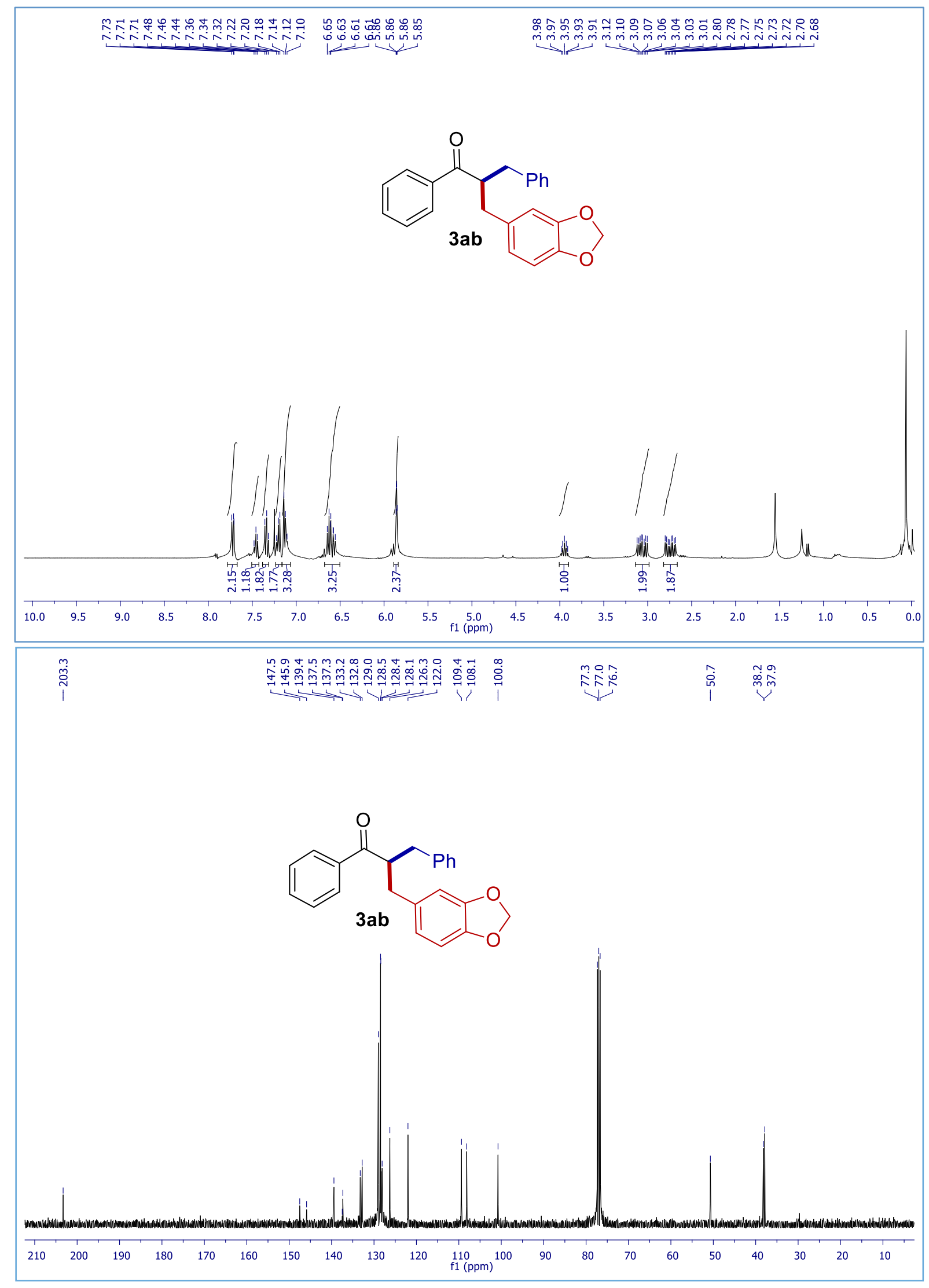




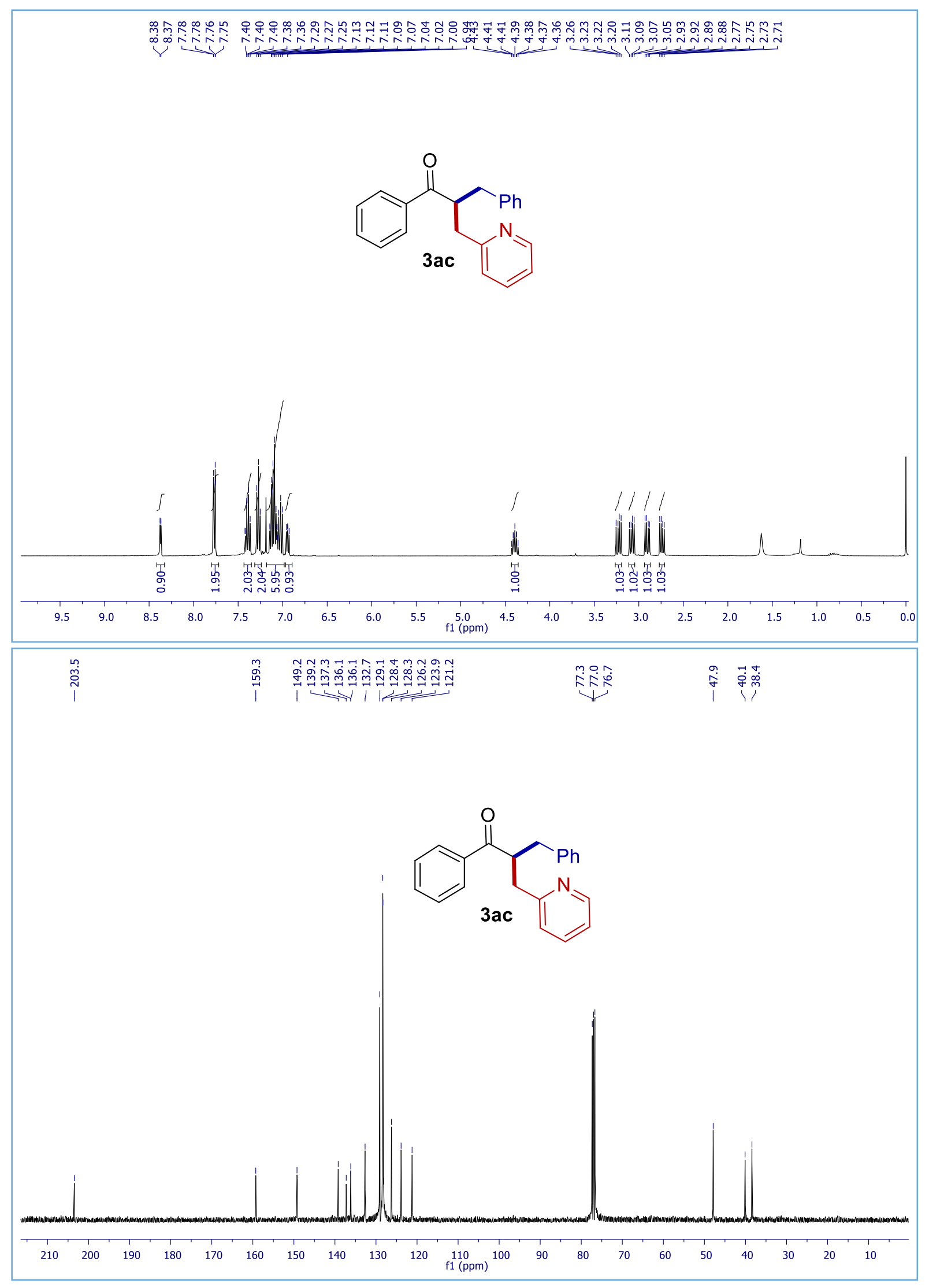

S46 


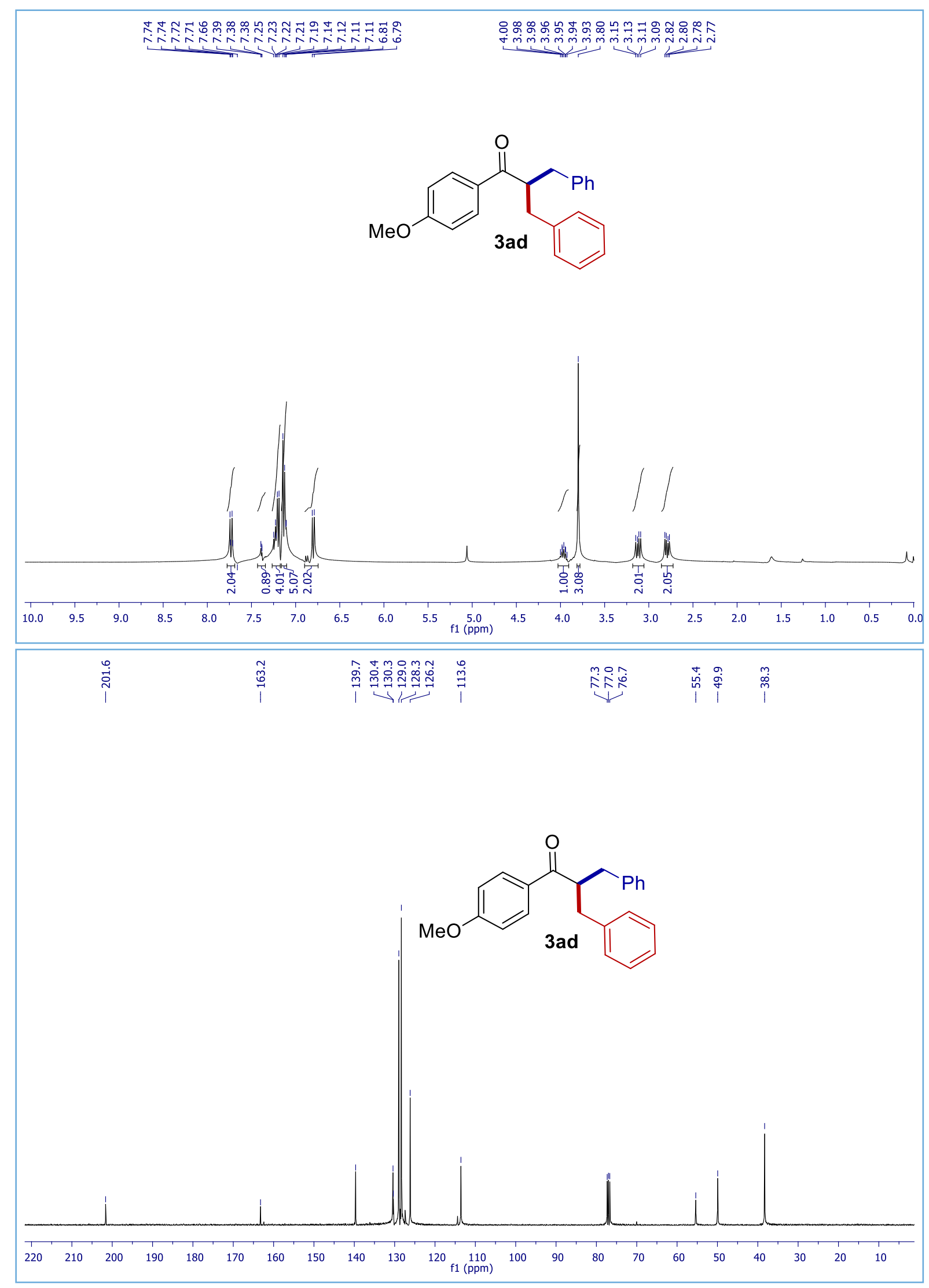



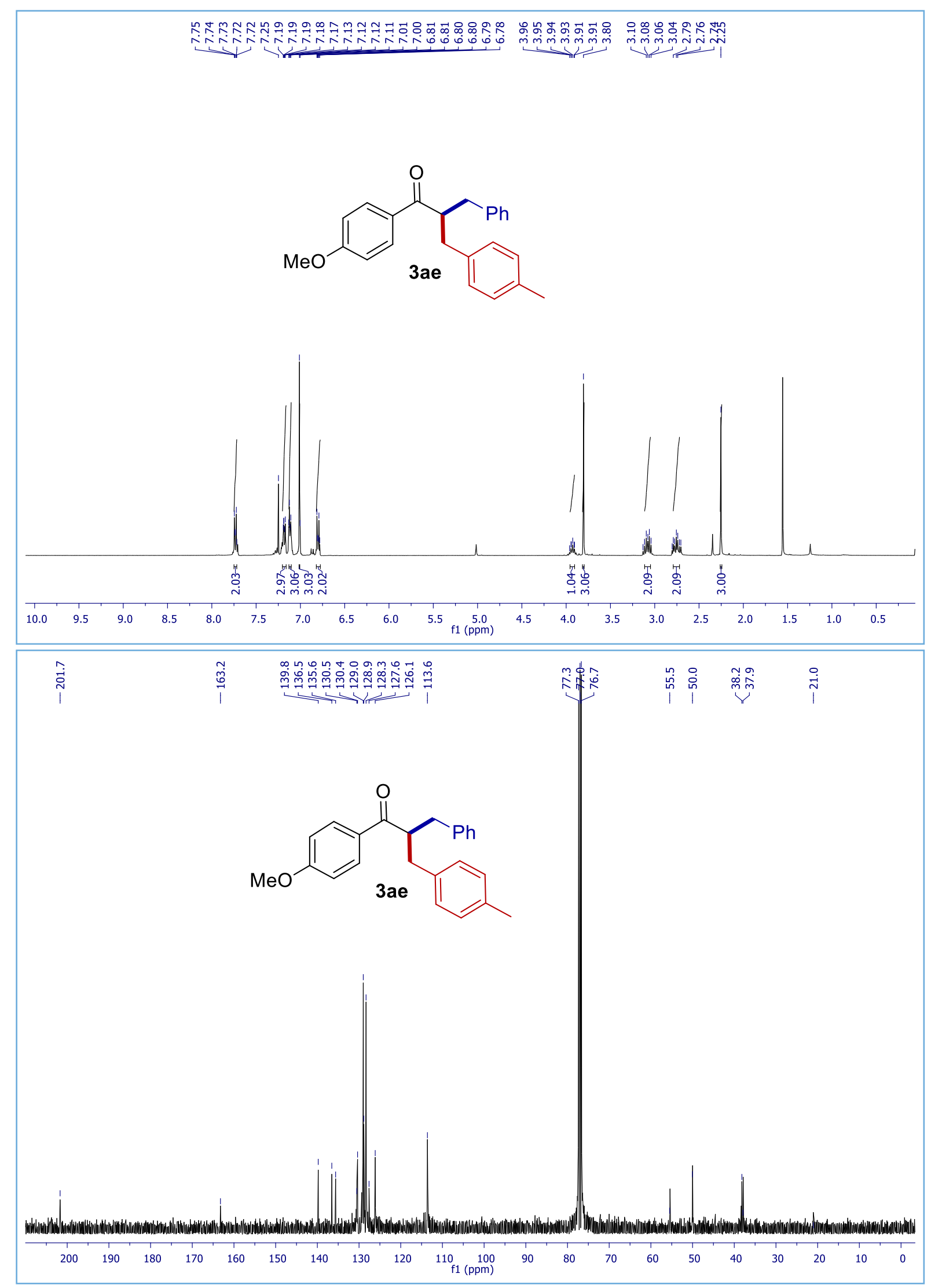


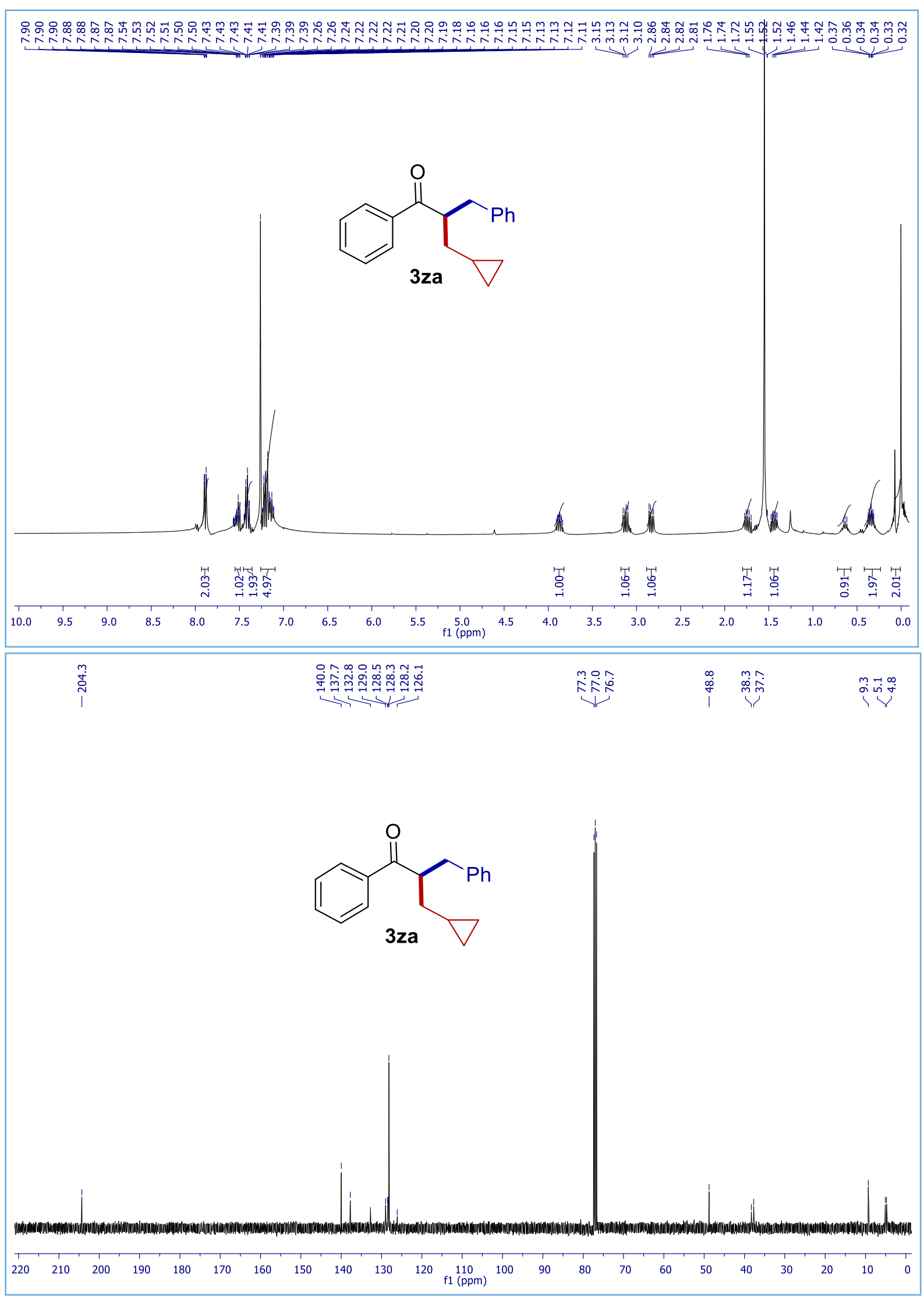



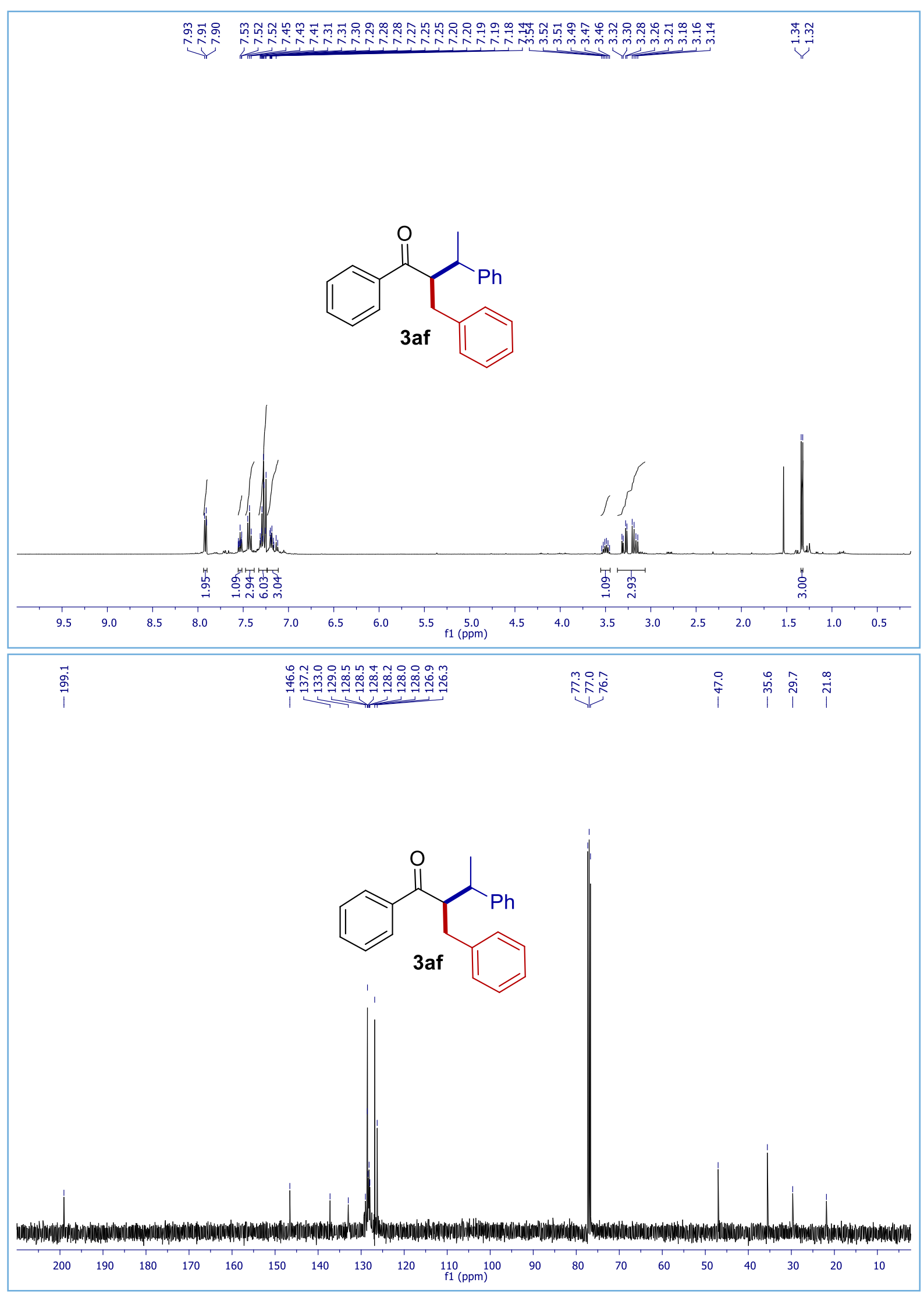


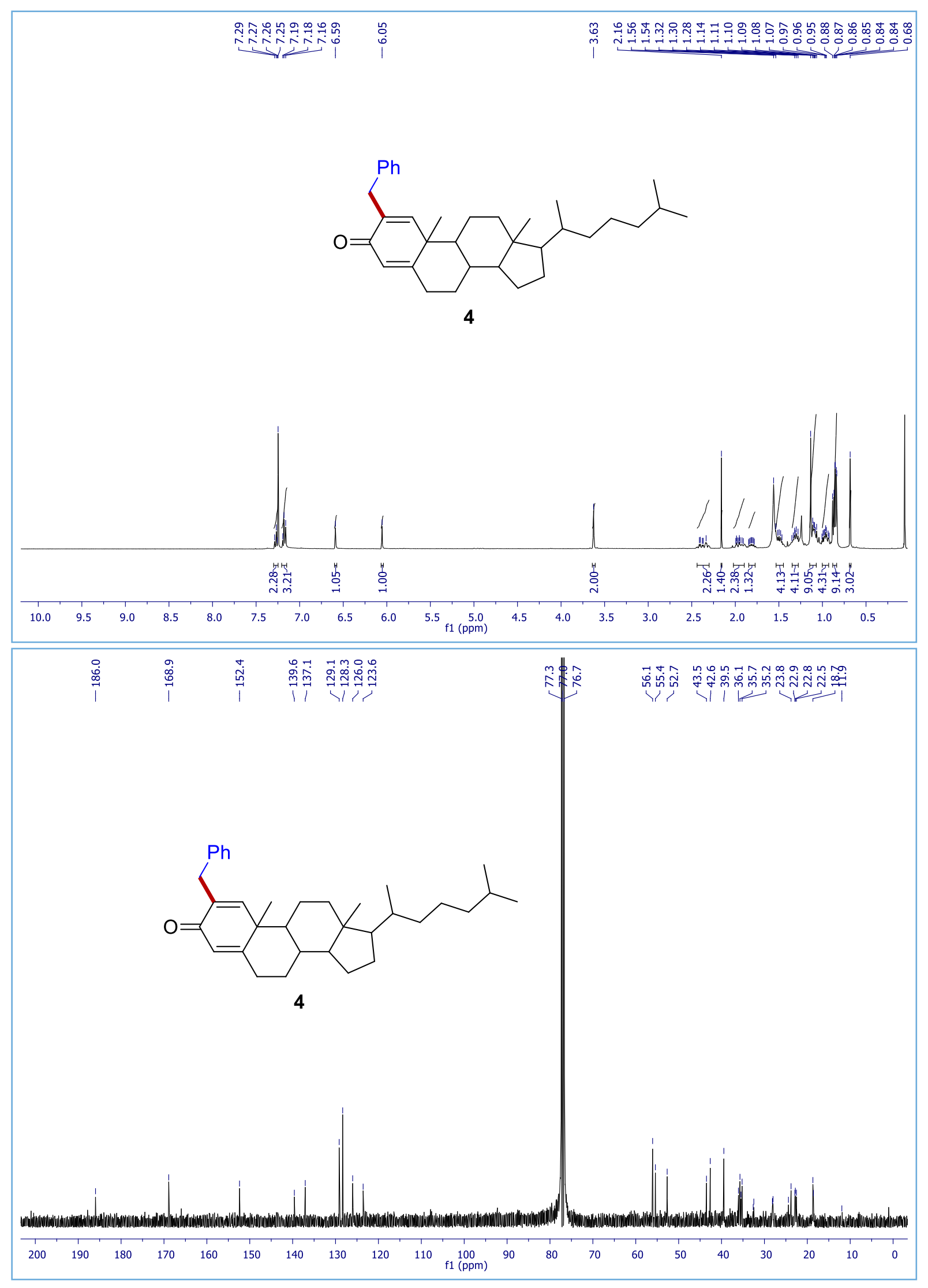

S51 


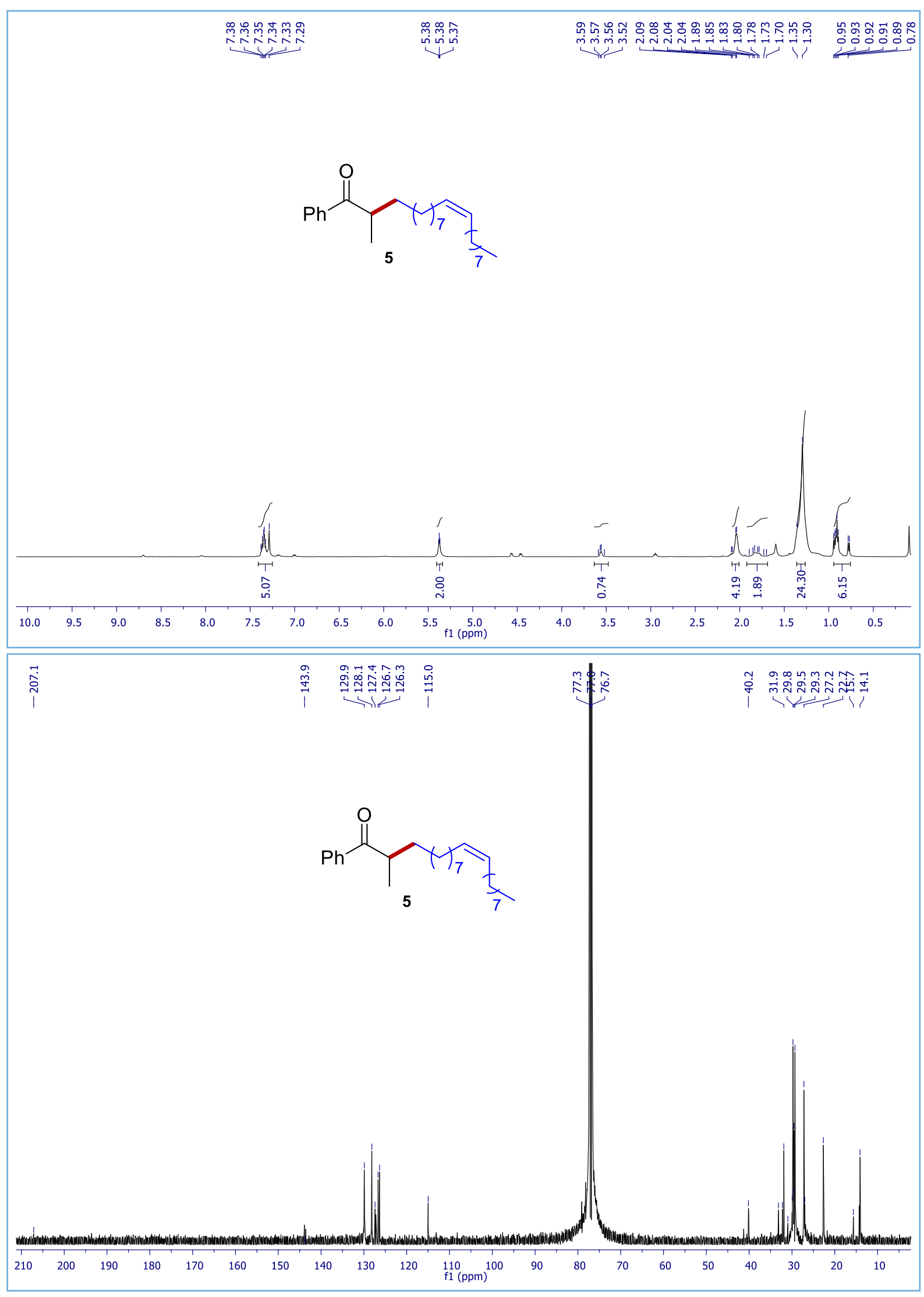



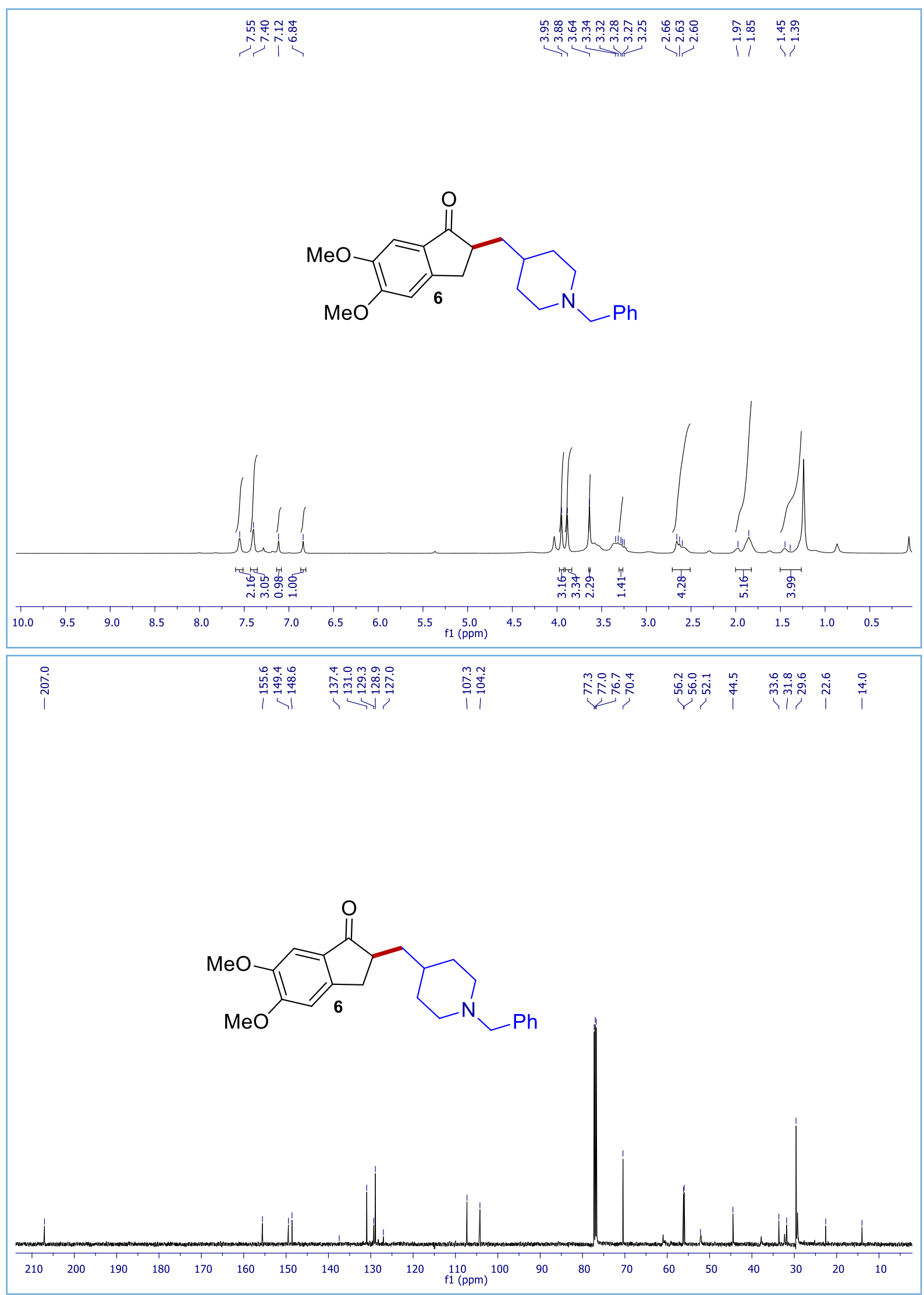


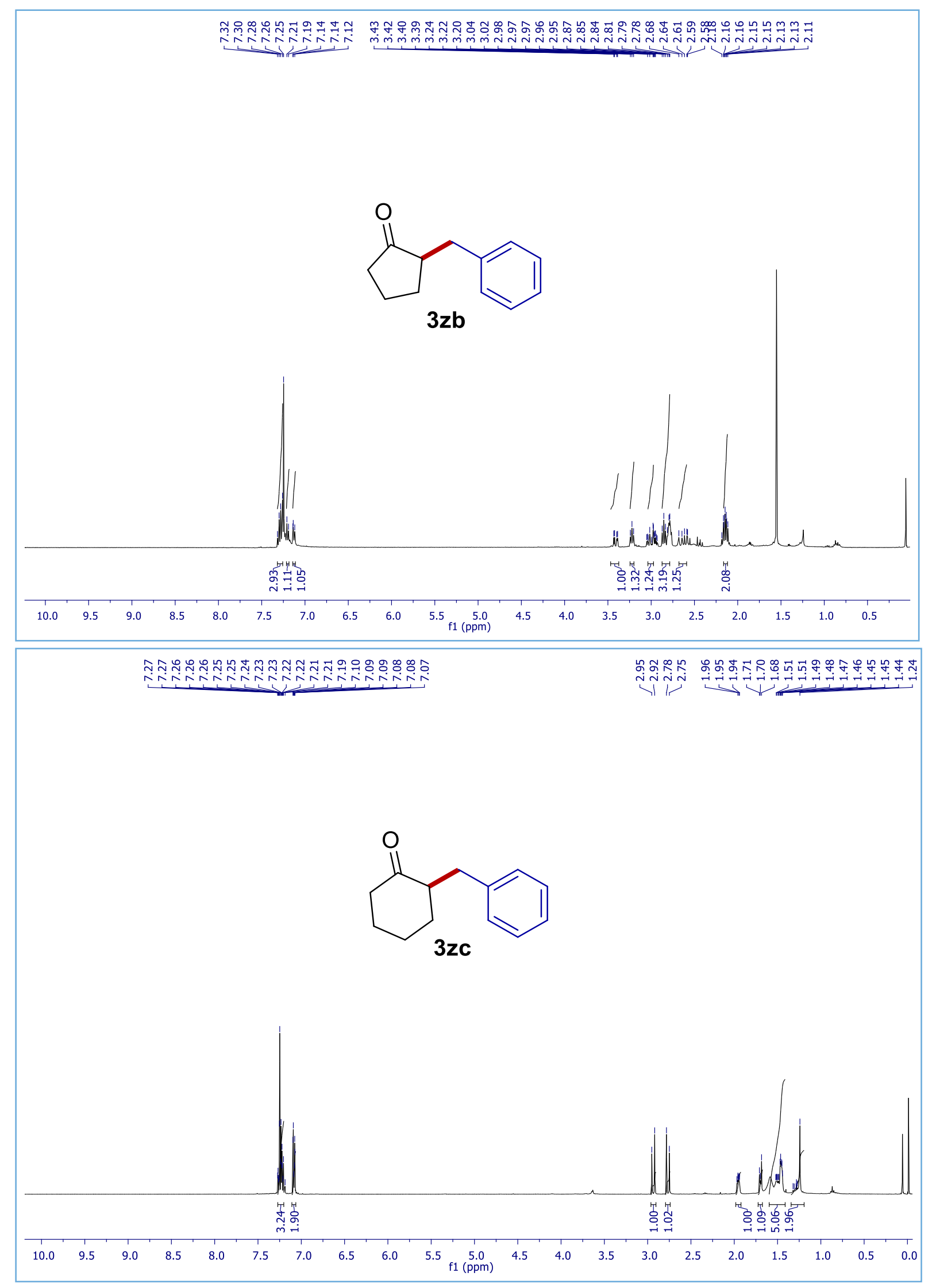




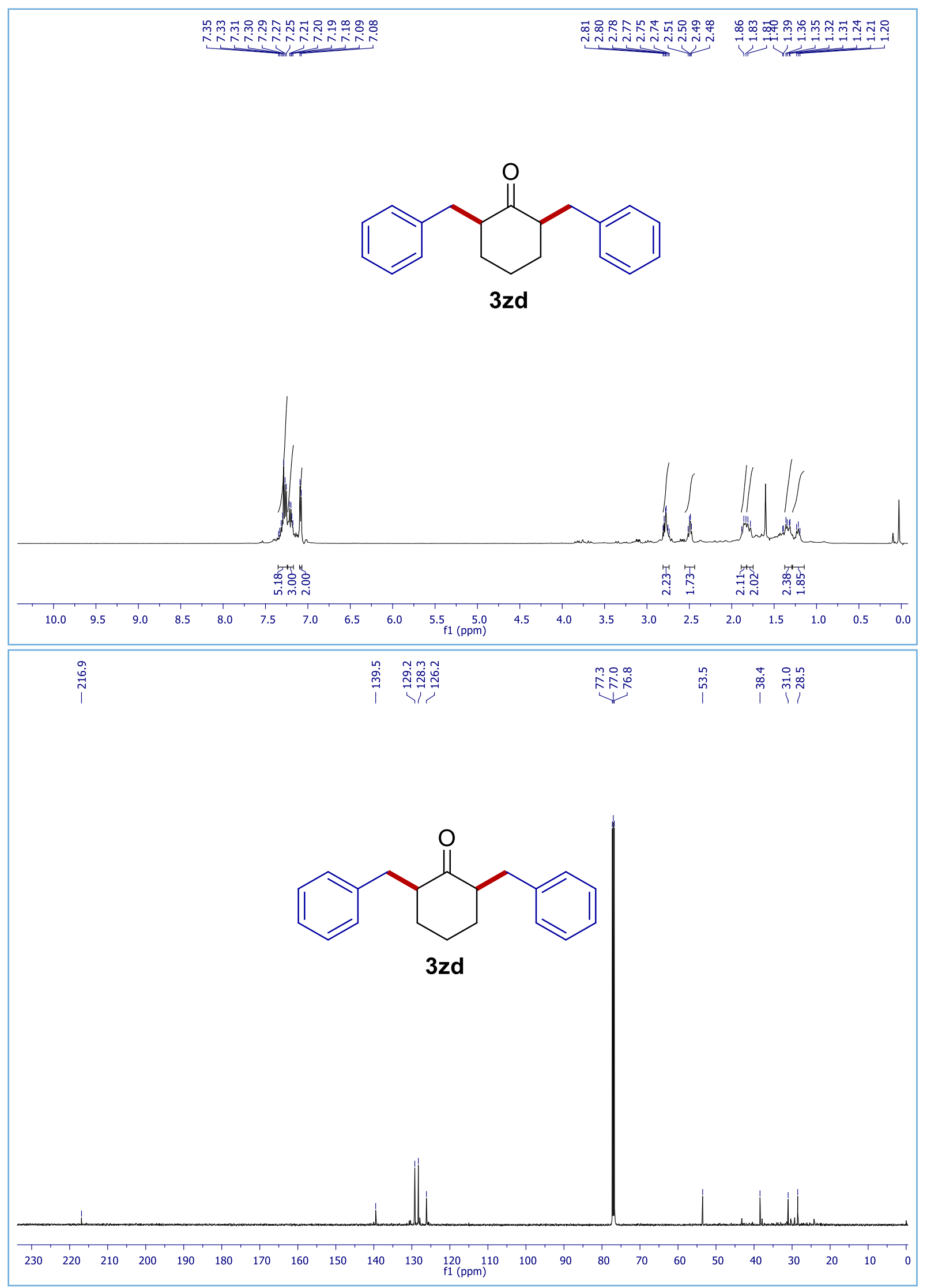



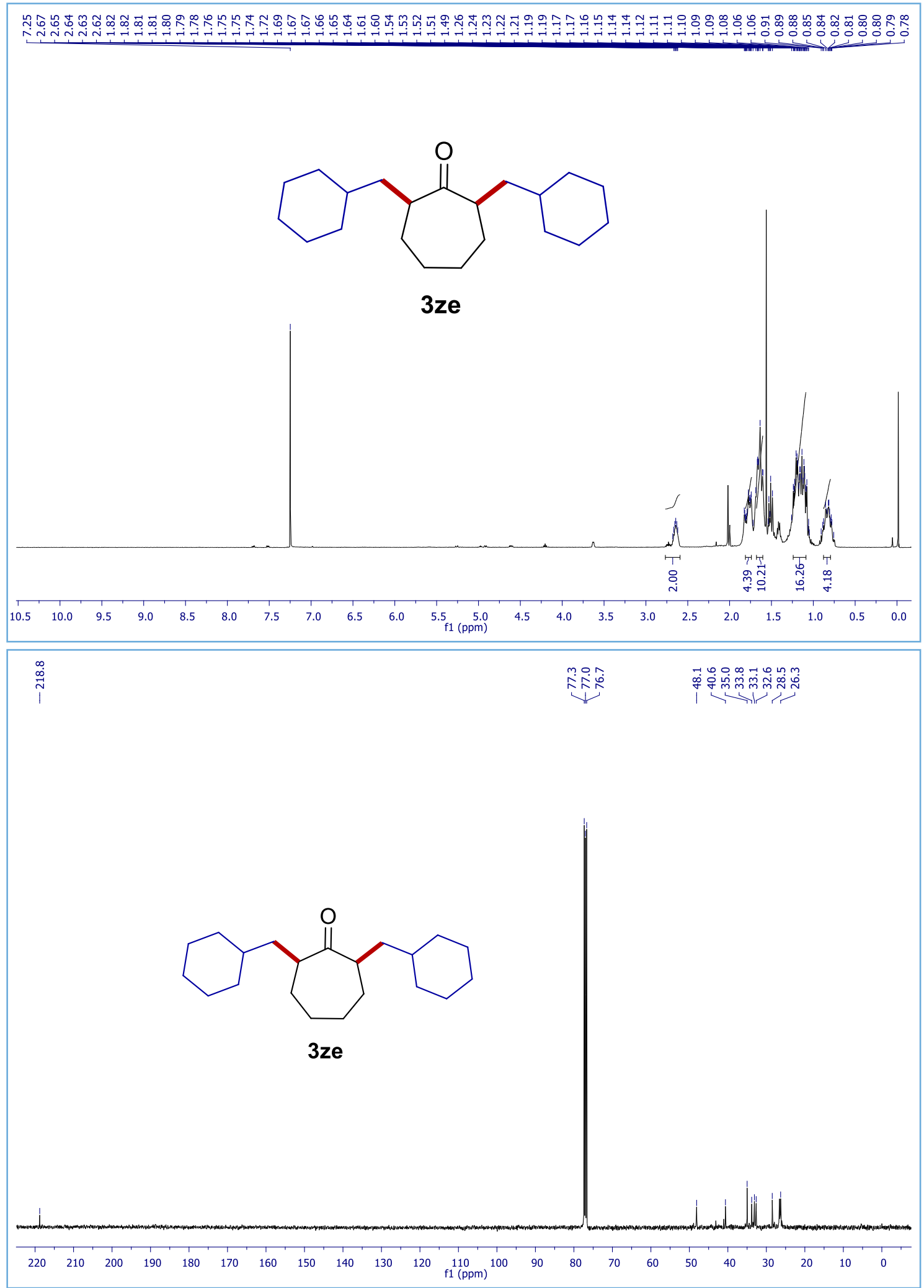